\title{
R-GROUP AND WHITTAKER SPACE OF SOME GENUINE REPRESENTATIONS
}

\author{
FAN GAO
}

\begin{abstract}
For a unitary unramified genuine principal series representation of a covering group, we study the associated R-group. We prove a formula relating the R-group to the dimension of the Whittaker space for the irreducible constituents of such a principal series representation. Moreover, for saturated covers of a semisimple simply-connected group, we also propose a simpler conjectural formula for such dimensions. This latter conjectural formula is verified in several cases, including covers of the symplectic groups.
\end{abstract}

\section{Contents}

1. Introduction 1

2. Covering group 6

3. Unitary unramified principal series 10

4. R-group 14

5. Whittaker space and the main conjecture 25

6. On the dimension of $\mathrm{Wh}_{\psi}\left(\pi_{\chi}^{u n}\right) \quad 33$

7. Covers of symplectic groups 37

8. Two remarks 44

$\begin{array}{ll}\text { References } & 47\end{array}$

\section{INTRODUCTION}

In [Gaob], we proposed and proved partially a conjectural formula for the dimension of a certain relative Whittaker space of the irreducible constituents of a regular unramified genuine principal series representation $I(\chi)$ of a covering group $\bar{G}$ over a non-archimedean field $F$. The formula in [Gaob] relates certain Kazhdan-Lusztig representations of the Weyl group to the dimensions of such relative Whittaker spaces.

This paper, as a companion to the above one, deals with the case where $I(\chi)$ is a unitary unramified genuine principal series, i.e., $\chi$ is a unitary unramified genuine character of the center $Z(\bar{T})$ of the covering torus $\bar{T} \subset \bar{G}$. In this case, $I(\chi)$ is a semisimple $\bar{G}$-module, and has a decomposition

$$
I(\chi)=\bigoplus_{\pi \in \Pi(\chi)} m_{\pi} \cdot \pi
$$

where $\pi$ 's are the non-equivalent constituents of $I(\chi)$. These representations $\pi$ thus should constitute an $L$-packet $\Pi(\chi)$. The reducibility of $I(\chi)$ and the above decomposition is controlled by a certain Knapp-Stein R-group $R_{\chi} \subset W_{\chi}$, where $W_{\chi} \subset W$ is the stabilizer subgroup of $\chi$ inside the Weyl group $W$. In particular, there is a correspondence

$$
\operatorname{Irr}\left(R_{\chi}\right) \longleftrightarrow \Pi(\chi), \quad \sigma \leftrightarrow \pi_{\sigma}
$$

2010 Mathematics Subject Classification. Primary 11F70; Secondary 22E50.

Key words and phrases. covering groups, R-groups, Whittaker functionals, scattering matrix, gamma factor, Plancherel measure. 
between the irreducible representations of $R_{\chi}$ and elements in $\Pi(\chi)$ such that $m_{\pi_{\sigma}}=$ $\operatorname{dim}(\sigma)$. Since $\chi$ is unramified, one can show that $R_{\chi}$ is abelian and therefore $m_{\pi_{\sigma}}=1$ for every $\sigma$.

It is well-known that genuine representations of covering groups could have highdimensional Whittaker space (i.e., the space of Whittaker functionals), see the introductions of [Gaob, GSS] for brief literature reviews. In particular, $\operatorname{dim} \mathrm{Wh}_{\psi}(I(\chi))$ increases as the degree of covering increases. In view of the correspondence in (1), it is a natural question to ask:

- How to determine $\operatorname{dim} \operatorname{Wh}_{\psi}\left(\pi_{\sigma}\right)$ in terms of $\sigma \in \operatorname{Irr}\left(R_{\chi}\right)$ ?

Our goal is first to prove a formula for $\operatorname{dim} \mathrm{Wh}_{\psi}\left(\pi_{\sigma}\right)$ for general $\bar{G}$. Second, for saturated covers of a semisimple simply-connected $G$, we propose a simpler conjectural formula for $\operatorname{dim} \mathrm{Wh}_{\psi}\left(\pi_{\sigma}\right)$ in terms of the character pairing of $\sigma$ and a certain permutation representation $\sigma^{\mathscr{X}}$ of $R_{\chi}$. We will also verify several cases of this conjectural formula in this paper.

1.1. Background and motivation. We briefly recall some relevant works for linear algebraic groups which motivate our consideration in this paper.

For a linear algebraic group $G$ and $\chi$ a character of $T$ (not necessarily unramified), the correspondence (1) arises from the theory of Harish-Chandra and Knapp-Stein for the commuting algebra $\operatorname{End}(I(\chi))$ of the principal series representation $I(\chi)$, especially from the algebra isomorphism

$$
\mathbf{C}\left[R_{\chi}\right] \simeq \operatorname{End}(I(\chi)) .
$$

In fact, for general parabolic induction (i.e., parabolically induced representation) for $G$, the theory of R-group was initiated in the work of Knapp-Stein for real groups [KS75]. Based on Harish-Chandra's work [Sil79b], Silberger worked out the formulation for $p$-adic groups [Sil78, Sil79a]; in particular, he showed that Harish-Chandra's commuting algebra theorem holds, which then gives an analogue of the isomorphism (2) above for general parabolic induction on linear algebraic groups. Here for simplicity in this introduction, we ignore the subtleties of the two-cocycle twisting in the algebra of the R-group for general parabolic inductions, see [Art93] for details. For minimal parabolic induction of a Chevalley group, the group $R_{\chi}$ was computed explicitly by Keys [Key82a]; the study in the unramified case was furthered in [Key82b].

In the minimal parabolic case, the connection of the R-group with the Langlands correspondence was elucidated in [Key87,KS88]. For example, let $\phi_{\chi}$ be the L-parameter associated to the character $\chi$; Keys showed that the component group $\mathcal{S}_{\phi_{\chi}}$ is isomorphic to $R_{\chi}$, and thus elements inside the $L$-packet $\Pi(\chi)$ are also naturally parametrized by $\operatorname{Irr}\left(\mathcal{S}_{\phi_{\chi}}\right)$. Beyond the principal series case, it was conjectured by Arthur [Art89] that the R-group associated with the parabolic induction $I(\sigma)$ from a discrete series $\sigma$ on the Levi subgroup is also isomorphic to the component group $\mathcal{S}_{\phi_{\sigma}}$, where $\phi_{\sigma}$ is the $L$-parameter associated to $I(\sigma)$. We refer to [BZ05, Gol11, BG12] and the references therein for works in this direction.

Such a relation between $\Pi(\chi)$ and $\phi_{\chi}$ demonstrates a prototype of the general idea of the local Langlands parametrization for admissible representations of a local reductive group $G$. Let $W_{F}^{\prime}=W_{F} \times \mathrm{SL}_{2}(\mathbf{C})$ be the Weil-Deligne group of $F$, where $W_{F}$ is the local Weil group. Let ${ }^{L} G$ be the $L$-group of $G$. For each parameter

$$
\phi: W_{F}^{\prime} \longrightarrow{ }^{L} G,
$$

the local conjecture of Langlands asserts that there is an $L$-packet $\Pi_{\phi}$ consisting of irreducible admissible representations of $G$ which satisfy certain desiderata, see [Bor79]. 
Members inside the same packet $\Pi_{\phi}$ are equipped with the same $L$-function and $\varepsilon$-factor. Moreover, as alluded to above, if $\phi$ is a tempered parameter (i.e., the image of $\left.\phi\right|_{W_{F}}$ in the dual group of $G$ is relatively compact), then the component group $\mathcal{S}_{\phi}$ conjecturally parametrizes elements in the $L$-packet $\Pi_{\phi}$ (see [Art06]), i.e., there is a bijection

$$
\operatorname{Irr}\left(\mathcal{S}_{\phi}\right) \longleftrightarrow \Pi_{\phi}
$$

The bijection in (3) originally manifests in the theory of endoscopic transfer, and in particular in the character identity relations matching orbital integrals arising from the transfers (cf. [LL79, She79, She82, Art84, Art93]). More generally, in order to deal with non-tempered representation in the global $L^{2}$ spectral decomposition, it was postulated by Arthur that one should consider a parameter $\varphi$ of $W_{F}^{\prime} \times \mathrm{SL}_{2}(\mathbf{C})$ valued in the Langlands $L$-group ${ }^{L} G$; the component group $\mathcal{S}_{\varphi}$ should parametrize certain Arthur-packet which plays a crucial role in the work of Arthur in formulating his global multiplicity formula for the discrete spectrum of automorphic representations of a linear reductive group (see [Art89, Art13]).

It should be noted that the bijection in (3) is not canonical, which from the geometric side depends on the normalization of the Langlands-Shelstad transfer factors, and from the representation-theoretic side on the normalization of intertwining operators (see [KS88, Sha83, Sha90]). Indeed, one can always twist the bijection by a character of the component group $\mathcal{S}_{\phi}$. However, if $\phi$ is a tempered parameter, then it was conjectured in [Sha90] that $\Pi_{\phi}$ always contains a unique generic element with respect to a fixed Whittaker datum of $G$. In particular, for a fixed Whittaker datum of $G$, there is a one-toone correspondence between generic tempered representations and tempered $L$-packets. Granted with this tempered-packet conjecture, the correspondence in (3) can then be normalized such that the unique generic element (with respect to the fixed Whittaker datum) is parametrized by $\mathbb{1} \in \operatorname{Irr}\left(\mathcal{S}_{\phi}\right)$. We refer the reader to [Sha11] and the references therein for works in this direction.

It is to this last problem on genericty of representations inside $\Pi_{\phi}$, where $\phi=\phi_{\chi}$ is the parameter for a unitary unramified genuine principal series $I(\chi)$ of a covering group, that our object in this paper pertains. Fix a Whittaker datum $(\bar{B}=\bar{T} U, \psi)$ for a covering group $\bar{G}$. The Whittaker space $\mathrm{Wh}_{\psi}(I(\chi))$ is understood as a consequence of Rodier's heridity and the fact that $\bar{T}$ has only trivial unipotent subgroup. However, if $I(\chi)$ is reducible, then for any constituent $\pi$, it is a natural but delicate question to determine the dimension $\operatorname{dim} \mathrm{Wh}_{\psi}(\pi)$.

For a unitary unramified genuine $\chi$ the correspondence (1) continues to hold. The proof is essentially the same as in the linear algebraic case, and relies on the covering analogue of (2), which follows from the recent work of W.-W. Li [Li12, Li14] and C.-H. Luo [Luo]. In fact, we will also show the isomorphism $R_{\chi} \simeq \mathcal{S}_{\phi_{\chi}}$. However, since it is possible to have $\operatorname{dim} \mathrm{Wh}_{\psi}(\pi)>0$ for every constituent $\pi$ of $I(\chi)$, there does not seem to be a preferred choice of $\pi \in \Pi(\chi)$ by using the genericity criterion. Thus, we choose the normalization for the correspondence in (1) such that the unique unramified constituent $\pi_{\chi}^{u n} \subset I(\chi)$ corresponds to the trivial representation $\mathbb{1}$ of $R_{\chi}$, i.e.,

$$
\pi_{\mathbb{1}}=\pi_{\chi}^{u n} .
$$

For unramified principal series of a linear algebraic group, this is the natural choice since $\pi_{\chi}^{u n}$ is generic with respect to the fixed Whittaker datum, as a consequence of the Casselman-Shalika formula.

With this normalized correspondence $\sigma \leftrightarrow \pi_{\sigma}$, we want to determine $\operatorname{dim} \mathrm{Wh}_{\psi}\left(\pi_{\sigma}\right)$ in terms of $\sigma$ for every $\sigma \in \operatorname{Irr}\left(R_{\chi}\right)$. We hope that the results in this paper will eventually find applications in the context of global automorphic representations for covering groups. 
1.2. Main conjecture. Let $\chi$ be a unitary unramified genuine character of $Z(\bar{T})$. Associated to $\bar{T}$, there is a finite abelian group

$$
\mathscr{X}_{Q, n}:=Y / Y_{Q, n},
$$

which is the quotient of the cocharacter lattice $Y$ of $G$ by a certain sublattice $Y_{Q, n}$. Here $\mathscr{X}_{Q, n}$ is the "moduli space" of $\mathrm{Wh}_{\psi}(I(\chi))$; in particular,

$$
\operatorname{dim} \mathrm{Wh}_{\psi}(I(\chi))=\left|\mathscr{X}_{Q, n}\right| \text {. }
$$

The group $\mathscr{X}_{Q, n}$ is endowed with a natural twisted $W$-action which we denote by w[y]. From this one has a permutation representation

$$
\sigma^{\mathscr{X}}: W \longrightarrow \operatorname{Perm}\left(\mathscr{X}_{Q, n}\right)
$$

given by $\sigma^{\mathscr{X}}(w)(y)=\mathbb{w}[y]$. Let $\mathcal{O}_{\mathscr{X}}$ be the set of all $W$-orbits (with respect to this twisted action) in $\mathscr{X}_{Q, n}$. Clearly, for each $W$-orbit $\mathcal{O}_{y} \in \mathcal{O}_{\mathscr{X}}$, one has the permutation representation

$$
\sigma_{\mathcal{O}_{y}}^{\mathscr{X}}: W \longrightarrow \operatorname{Perm}\left(\mathcal{O}_{y}\right)
$$

moreover, $\sigma^{\mathscr{X}}$ decomposes as a sum of all $\sigma_{\mathcal{O}_{y}}^{\mathscr{X}}$, i.e.,

$$
\sigma^{\mathscr{X}}=\bigoplus_{\mathcal{O}_{y} \in \mathcal{O}_{\mathscr{X}}} \sigma_{\mathcal{O}_{y}}^{\mathscr{X}} .
$$

By restriction, $\sigma_{\mathcal{O}_{y}}^{\mathscr{X}}$ could be viewed as a permutation representation of $R_{\chi} \subset W_{\chi} \subset W$.

For every $W$-orbit $\mathcal{O}_{y} \subset \mathscr{X}_{Q, n}$, there is also a natural subspace $\mathrm{Wh}_{\psi}\left(\pi_{\sigma}\right)_{\mathcal{O}_{y}} \subset \mathrm{Wh}_{\psi}\left(\pi_{\sigma}\right)$ (see (29)) such that

$$
\mathrm{Wh}_{\psi}\left(\pi_{\sigma}\right)=\bigoplus_{\mathcal{O}_{y} \in \mathcal{O}_{\mathscr{X}}} \mathrm{Wh}_{\psi}\left(\pi_{\sigma}\right)_{\mathcal{O}_{y}} .
$$

Conjecture 1.1 (Conjecture 5.3). Let $\bar{G}$ be a saturated $n$-fold cover (see Definition 2.1) of a semisimple simply-connected group $G$. In the normalized correspondence $\operatorname{Irr}\left(R_{\chi}\right) \longleftrightarrow$ $\Pi(\chi), \sigma \leftrightarrow \pi_{\sigma}$ such that $\pi_{\mathbb{1}}=\pi_{\chi}^{u n}$, one has

$$
\operatorname{dim} \mathrm{Wh}_{\psi}\left(\pi_{\sigma}\right)_{\mathcal{O}_{y}}=\left\langle\sigma, \sigma_{\mathcal{O}_{y}}^{\mathscr{X}}\right\rangle_{R_{\chi}}
$$

for every orbit $\mathcal{O}_{y} \in \mathcal{O}_{\mathscr{X}}$, where $\langle-,-\rangle_{R_{\chi}}$ denotes the pairing of two representations of $R_{\chi}$. Consequently,

$$
\operatorname{dim} \mathrm{Wh}_{\psi}\left(\pi_{\sigma}\right)=\left\langle\sigma, \sigma^{\mathscr{X}}\right\rangle_{R_{\chi}}
$$

for every $\sigma \in \operatorname{Irr}\left(R_{\chi}\right)$; in particular, $\operatorname{dim} \mathrm{Wh}_{\psi}\left(\pi_{\chi}^{u n}\right)$ is equal to the number of $R_{\chi}$-orbits in $\mathscr{X}_{Q, n}$.

1.3. Main results. Prior to the formulation of the above conjecture, a substantial part of the paper is devoted to analyzing the group $R_{\chi}$ and proving for covers of general reductive groups an unconditional formula for $\operatorname{dim} \mathrm{Wh}_{\psi}\left(\pi_{\sigma}\right)_{\mathcal{O}_{y}}$ in terms of $\sigma$ and a certain representation $\sigma_{\mathcal{O}_{y}}^{\mathrm{Wh}}$ of $R_{\chi}$. We briefly outline the content of the paper and highlight some of our results.

After a brief introduction on covering groups in $\S 2$, we study in $\S 3$ the normlized intertwining operator between genuine principal series of $\bar{G}$. As in the case for linear algebraic groups, the normalization is given by the Langlands $L$-functions, and one important property is the cocycle relation of the normalized intertwining operators which does not depend on the length function of $W$.

In $\S 4$, we analyze the group $R_{\chi}$ based on the work of Keys [Key82a, Key87], W.W. Li [Li12, Li14] and C.-H. Luo [Luo]. In particular, it follows from [Luo] that for a unitary unramified genuine principal series $I(\chi)$, one has an algebra isomorphism $\mathbf{C}\left[R_{\chi}\right] \simeq$ 
$\operatorname{End}(I(\chi))$. We show how to compute $R_{\chi}$ by relating it to another group $R_{\chi}^{s c}$, which is equal to the R-group of a certain unramified principal series of a simply-connected Chevalley group $H^{s c}$. The group $R_{\chi}^{s c}$ is explicitly determined in Keys' paper [Key82a, Key82b] for principal series of simply-connected Chevalley groups. Moreover, by reducing to the linear algebraic case, we prove in Theorem 4.9 the isomorphism $R_{\chi} \simeq \mathcal{S}_{\phi_{\chi}}$, where $\phi_{\chi}$ is the $L$-parameter of $I(\chi)$ valued in the $L$-group of $\bar{G}$ constructed by M. Weissman [Wei18].

Denote by $\bar{G}^{\vee}$ (resp. ${ }^{L} \bar{G}$ ) the dual group (resp. $L$-group) for the covering group $\bar{G}$. The following is an amalgam of Proposition 4.4, Theorem 4.6 and Theorem 4.9.

Theorem 1.2. Let $\bar{G}$ be an $n$-fold cover of a linear algebraic group $G$. Let $\chi$ be a unitary unramified genuine character of $Z(\bar{T})$. We have:

- $R_{\chi} \subseteq R_{\chi}^{s c}$ with $R_{\chi}^{s c}$ being an abelian group, and if $\bar{G}$ is semisimple, then $\left[R_{\chi}^{s c}\right.$ : $\left.R_{\chi}\right] \leq\left|Z\left(\bar{G}^{\vee}\right)\right|$, where $Z\left(\bar{G}^{\vee}\right)$ is the center of $\bar{G}^{\vee}$;

- $R_{\chi} \simeq \mathcal{S}_{\phi_{\chi}}$, where $\mathcal{S}_{\phi_{\chi}}$ is the component group of the parameter $\phi_{\chi}: W_{F} \rightarrow{ }^{L} \bar{G}$.

We remark that since $\chi$ is unitary, the parameter $\phi_{\chi}$ is trivial on $\mathrm{SL}_{2}(\mathbf{C}) \subset W_{F}^{\prime}$, and thus it suffices to consider the Weil group $W_{F}$ alone.

Section $\S 5$ is devoted to stating and investigating several aspects of the main Conjecture 5.3, i.e., Conjecture 1.1 above. First, we show that for every $\mathcal{O}_{y} \in \mathcal{O}_{\mathscr{X}}$, the space $\mathrm{Wh}_{\psi}(I(\chi))_{\mathcal{O}_{y}}$ affords a natural representation

$$
\sigma_{\mathcal{O}_{y}}^{\mathrm{Wh}}: R_{\chi} \longrightarrow \mathrm{GL}\left(\mathbf{C}^{\left|\mathcal{O}_{y}\right|}\right)
$$

such that the following holds (cf. Theorem 5.6):

Theorem 1.3. Let $\bar{G}$ be an $n$-fold cover of a connected reductive group $G$. For every orbit $\mathcal{O}_{y} \in \mathcal{O}_{\mathscr{X}}$, one has

$$
\operatorname{dim} \mathrm{Wh}_{\psi}\left(\pi_{\sigma}\right)_{\mathcal{O}_{y}}=\left\langle\sigma, \sigma_{\mathcal{O}_{y}}^{\mathrm{Wh}}\right\rangle_{R_{\chi}} .
$$

Consequently, $\operatorname{dim} \mathrm{Wh}_{\psi}\left(\pi_{\sigma}\right)=\left\langle\sigma, \sigma^{\mathrm{Wh}}\right\rangle_{R_{\chi}}$.

Here $\sigma_{\mathcal{O}_{y}}^{\mathrm{Wh}}(\mathbb{w})$ is represented by the matrix $\gamma(w, \chi) \cdot \mathcal{S}_{\mathfrak{R}}(w, i(\chi))$, where $\gamma(w, \chi)$ is the gamma-factor associated to $\mathbb{w}$ and $\mathcal{S}_{\mathfrak{R}}(w, i(\chi))$ a so-called scattering matrix. As an application of the above theorem, we show in $§ 5.5$ that a result of D. Szpruch [Szp15] on double cover of $\mathrm{GSp}_{2 r}$ could be recovered from it, see Theorem 5.10. Here Theorem 1.3 also implies that Conjecture 1.1 is equivalent to the following (cf. Conjecture 5.7):

Conjecture 1.4. Let $\bar{G}$ be a saturated $n$-fold cover of a semisimple simply-connected $G$. Then for every orbit $\mathcal{O}_{y} \subset \mathscr{X}_{Q, n}$, one has $\sigma_{\mathcal{O}_{y}}^{\mathrm{Wh}}=\sigma_{\mathcal{O}_{y}}^{\mathscr{X}}$; or equivalently,

$$
\operatorname{Tr}\left(\mathcal{S}_{\mathfrak{R}}(w, i(\chi))\right)=\left|\left(\mathcal{O}_{y}\right)^{\mathbb{w}}\right| \cdot \gamma(w, \chi)^{-1}
$$

for every $\mathbb{w} \in R_{\chi}$.

Using the formulation in Conjecture 1.4, we prove several results in $\S 6$ :

- For a general reductive group $G$, we show that there is an exceptional set $\mathscr{X}_{Q, n}^{\text {exc }} \subset$ $\left(\mathscr{X}_{Q, n}\right)^{W}$, which might be empty, such that $\sigma_{\mathcal{O}_{y}}^{\text {Wh }}=\sigma_{\mathcal{O}_{y}}^{\mathscr{X}}=\mathbb{1}_{R_{\chi}}$ for $y \in \mathscr{X}_{Q, n}^{\text {exc }}$. It follows that $\operatorname{dim} \mathrm{Wh}_{\psi}\left(\pi_{\chi}^{u n}\right) \geq\left|\mathscr{X}_{Q, n}^{\mathrm{exc}}\right|$; it also implies that Conjecture 1.4 holds for such $\mathcal{O}_{y}$. This is the content of Theorem 6.1. 
- In $\S 6.2$, we consider the Whittaker space $\mathrm{Wh}_{\psi}\left(\pi_{\chi}^{u n}\right)$ from the perspective of unramified Whittaker functions. Using an analogue of Casselman-Shalika formula proved in [GSS], we show in Theorem 6.5 a result on $\operatorname{dim} \mathrm{Wh}_{\psi}\left(\pi_{\chi}^{u n}\right)$, which is compatible with Theorem 6.1.

In $\S 7$, we verify the following:

Theorem 1.5 (Theorem 7.1). Conjecture 1.4 (and thus Conjecture 1.1) holds for the $n$-fold covers $\overline{\mathrm{Sp}}_{2 r}^{(n)}$.

We also prove in $\S 7$ that Conjecture 1.1 holds for the double cover of $\mathrm{SL}_{3}$ by explicit computations.

Lastly, in $\S 8$, we consider $n$-fold covers of $\mathrm{SO}_{3}$ and the double cover of $\mathrm{Spin}_{6} \simeq \mathrm{SL}_{4}$, and show that the naive analogue of Conjecture 1.4 fails for such covers. Thus, the constraints on $G$ being simply-connected and on $\bar{G}$ being saturated seem to be indispensable. For general reductive groups, a unified conjectural formula for $\mathrm{Wh}_{\psi}\left(\pi_{\sigma}\right)_{\mathcal{O}_{y}}$ in terms of $\sigma_{\mathcal{O}_{y}}^{\mathscr{X}}$ involves subtleties beyond the consideration in this paper, and its intimate relation with the R-group has yet to be unveiled in full generality.

\section{Covering group}

Our exposition on covering group is essentially the same as in [Gaob, §2]. However, to ensure that the paper is self-contained, we will briefly recall again some summarized results on $\bar{G}$.

Let $F$ be a finite extension of $\mathbf{Q}_{p}$. Denote by $O \subset F$ the ring of integers of $F$ and $\varpi \in O$ a fixed uniformizer.

2.1. Covering group. Let $\mathbf{G}$ be a split connected linear algebraic group over $F$ with a maximal split torus $\mathbf{T}$. Let

$$
\left\{X, \Delta, \Phi ; Y, \Delta^{\vee}, \Phi^{\vee}\right\}
$$

be the based root datum of $\mathbf{G}$. Here $X$ (resp. $Y$ ) is the character lattice (resp. cocharacter lattice) for $(\mathbf{G}, \mathbf{T})$. Choose a set $\Delta \subseteq \Phi$ of simple roots from the set of roots $\Phi$, and let $\Delta^{\vee}$ be the corresponding simple coroots from $\Phi^{\vee}$. This gives us a choice of positive roots $\Phi_{+}$and positive coroots $\Phi_{+}^{\vee}$. Write $Y^{\text {sc }} \subseteq Y$ for the sublattice generated by $\Phi^{\vee}$. Let $\mathbf{B}=\mathbf{T} \mathbf{U}$ be the Borel subgroup associated with $\Delta$. Denote by $\mathbf{U}^{-} \subset \mathbf{G}$ the unipotent subgroup opposite $\mathbf{U}$.

Fix a Chevalley-Steinberg system of pinnings for $(\mathbf{G}, \mathbf{T})$. That is, we fix a set of compatible isomorphisms

$$
\left\{e_{\alpha}: \mathbf{G}_{\mathrm{a}} \rightarrow \mathbf{U}_{\alpha}\right\}_{\alpha \in \Phi},
$$

where $\mathbf{U}_{\alpha} \subseteq \mathbf{G}$ is the root subgroup associated with $\alpha$. In particular, for each $\alpha \in \Phi$, there is a unique homomorphism $\varphi_{\alpha}: \mathbf{S L}_{2} \rightarrow \mathbf{G}$ which restricts to $e_{ \pm \alpha}$ on the upper and lower triangular subgroup of unipotent matrices of $\mathbf{S L}_{2}$, respectively.

Denote by $W$ the Weyl group of $(\mathbf{G}, \mathbf{T})$, which we identify with the Weyl group of the coroot system. In particular, $W$ is generated by simple reflections $\left\{\mathbb{w}_{\alpha}: \alpha^{\vee} \in \Delta^{\vee}\right\}$ in $Y \otimes \mathbf{Q}$. Let $l: W \rightarrow \mathbf{N}$ be the length function. Let $\mathbb{w}_{G}$ be the longest element in $W$.

Consider the algebro-geometric $\mathbf{K}_{2}$-extension $\overline{\mathbf{G}}$ of $\mathbf{G}$ studied by Brylinski and Deligne [BD01], which is categorically equivalent to the pairs $\{(D, \eta)\}$ (see $[G G 18, \S 2.6])$. Here $\eta: Y^{\mathrm{sc}} \rightarrow F^{\times}$is a homomorphism. On the other hand,

$$
D: Y \times Y \rightarrow \mathbf{Z}
$$

is a (not necessarily symmetric) bilinear form on $Y$ such that

$$
Q(y):=D(y, y)
$$


is a Weyl-invariant integer-valued quadratic form on $Y$. We call $D$ a bisector. Let $B_{Q}$ be the Weyl-invariant bilinear form associated to $Q$ by

$$
B_{Q}\left(y_{1}, y_{2}\right)=Q\left(y_{1}+y_{2}\right)-Q\left(y_{1}\right)-Q\left(y_{2}\right) .
$$

Clearly, $D\left(y_{1}, y_{2}\right)+D\left(y_{2}, y_{1}\right)=B_{Q}\left(y_{1}, y_{2}\right)$. Any $\overline{\mathbf{G}}$ is, up to isomorphism, incarnated by (i.e., categorically associated to) a pair $(D, \eta)$ for a bisector $D$ and $\eta$.

The couple $(D, \eta)$ play the following role for the structure of $\overline{\mathbf{G}}$.

- First, the group $\overline{\mathbf{G}}$ splits canonically and uniquely over any unipotent subgroup of $\mathbf{G}$. For $\alpha \in \Phi$ and $a \in \mathbf{G}_{a}$, denote by $\bar{e}_{\alpha}(a) \in \overline{\mathbf{G}}$ the canonical lifting of $e_{\alpha}(a) \in \mathbf{G}$. For $\alpha \in \Phi$ and $a \in \mathbf{G}_{m}$, define

$w_{\alpha}(a):=e_{\alpha}(a) \cdot e_{-\alpha}\left(-a^{-1}\right) \cdot e_{\alpha}(a)$ and $\bar{w}_{\alpha}(a):=\bar{e}_{\alpha}(a) \cdot \bar{e}_{-\alpha}\left(-a^{-1}\right) \cdot \bar{e}_{\alpha}(a)$.

This gives natural representatives $w_{\alpha}:=w_{\alpha}(1)$ in $\mathbf{G}$ and also $\bar{w}_{\alpha}:=\bar{w}_{\alpha}(1)$ in $\overline{\mathbf{G}}$, of the Weyl element $\mathbb{w}_{\alpha} \in W$. Moreover, for $h_{\alpha}(a):=\alpha^{\vee}(a) \in \mathbf{T}$, there is a natural lifting

$$
\bar{h}_{\alpha}(a):=\bar{w}_{\alpha}(a) \cdot \bar{w}_{\alpha}(-1) \in \overline{\mathbf{T}},
$$

which depends only on the pinnings and the canonical unipotent splittings.

- Second, there is a section $\mathbf{s}$ of $\overline{\mathbf{T}}$ over $\mathbf{T}$ such that the group law on $\overline{\mathbf{T}}$ includes the relation

$$
\mathbf{s}\left(y_{1}(a)\right) \cdot \mathbf{s}\left(y_{2}(b)\right)=\{a, b\}^{D\left(y_{1}, y_{2}\right)} \cdot \mathbf{s}\left(y_{1}(a) \cdot y_{2}(b)\right)
$$

for any $a, b \in \mathbf{G}_{m}$. Moreover, for $\alpha \in \Delta$ and the natural lifting $\bar{h}_{\alpha}(a)$ of $h_{\alpha}(a)$ above, one has

$$
\bar{h}_{\alpha}(a)=\left\{\eta\left(\alpha^{\vee}\right), a\right\} \cdot \mathbf{s}\left(h_{\alpha}(a)\right) \in \overline{\mathbf{T}}
$$

- Third, let $w_{\alpha} \in \mathbf{G}$ be the above natural representative of $\mathbb{w}_{\alpha} \in W$. For every $\overline{y(a)} \in \overline{\mathbf{T}}$ with $y \in Y$ and $a \in \mathbf{G}_{m}$, one has

$$
w_{\alpha} \cdot \overline{y(a)} \cdot w_{\alpha}^{-1}=\overline{y(a)} \cdot \bar{h}_{\alpha}\left(a^{-\langle y, \alpha\rangle}\right)
$$

where $\langle-,-\rangle$ is the paring between $Y$ and $X$.

If the derived subgroup of $\mathbf{G}$ is simply-connected, then the isomorphism class of $\overline{\mathbf{G}}$ is determined by the Weyl-invariant quadratic form $Q$. In particular, for such $\mathbf{G}$, any extension $\overline{\mathbf{G}}$ is incarnated by $(D, \eta=\mathbb{1})$ for some bisector $D$, up to isomorphism. In this paper, we assume that the composite

$$
\eta_{n}: Y^{s c} \rightarrow F^{\times} \rightarrow F^{\times} /\left(F^{\times}\right)^{n}
$$

of $\eta$ with the obvious quotient is trivial. For some consequence of this assumption, see $\S 2.2$ and the beginning of $\S 3$.

Let $n \in \mathbf{N}$. We assume that $F$ contains the full group of $n$-th roots of unity, denoted by $\mu_{n}$. An $n$-fold cover of $\mathbf{G}$, in the sense of [Wei18, Definition 1.2], is just a pair $(n, \overline{\mathbf{G}})$. The $\mathbf{K}_{2}$-extension $\overline{\mathbf{G}}$ gives rise to an $n$-fold covering $\bar{G}$ as follows. Let

$$
(-,-)_{n}: F \times F \rightarrow \mu_{n}
$$

be the $n$-th Hilbert symbol. The cover $\bar{G}$ arises from the central extension

$$
\mathbf{K}_{2}(F) \longleftrightarrow \overline{\mathbf{G}}(F) \stackrel{\phi}{\longrightarrow} \mathbf{G}(F)
$$

by push-out via the natural map $\mathbf{K}_{2}(F) \rightarrow \mu_{n}$ given by $\{a, b\} \mapsto(a, b)_{n}$. This gives

$$
\mu_{n} \longleftrightarrow \bar{G} \stackrel{\phi}{\longrightarrow} G \text {. }
$$

We may write $\bar{G}^{(n)}$ for $\bar{G}$ to emphasize the degree of covering. 
For any subset $H \subset G$, denote $\bar{H}:=\phi^{-1}(H)$. The relations for $\overline{\mathbf{G}}$ described above give rise to the corresponding relations for $\bar{G}$. For example, inherited from (4) is the following relation on $\bar{T}$,

$$
\mathbf{s}\left(y_{1}(a)\right) \cdot \mathbf{s}\left(y_{2}(b)\right)=(a, b)_{n}^{D\left(y_{1}, y_{2}\right)} \cdot \mathbf{s}\left(y_{1}(a) \cdot y_{2}(b)\right),
$$

where $y_{i} \in Y$ and $a, b \in F^{\times}$. The commutator $\left[\bar{t}_{1}, \bar{t}_{2}\right]:=\bar{t}_{1} \bar{t}_{2} \bar{t}_{1}^{-1} \bar{t}_{2}^{-1}$ of $\bar{T}$, which descends to a map $[-,-]: T \times T \rightarrow \mu_{n}$, is thus given by

$$
\left[y_{1}(a), y_{2}(b)\right]=(a, b)_{n}^{B_{Q}\left(y_{1}, y_{2}\right)} .
$$

A representation of $\bar{G}$ is called $\epsilon$-genuine (or simply genuine) if $\mu_{n}$ acts by a fixed embedding $\epsilon: \mu_{n} \hookrightarrow \mathbf{C}^{\times}$. We consider only genuine representations of a covering group in this paper.

Let $W^{\prime} \subset \bar{G}$ be the group generated by $\bar{w}_{\alpha}$ for all $\alpha$. Then the map $\bar{w}_{\alpha} \mapsto \mathbb{w}_{\alpha}$ gives a surjective homomorphism

$$
W^{\prime} \rightarrow W
$$

with kernel being a finite group. For any $\mathbb{w}=\mathbb{w}_{\alpha_{k}} \ldots \mathbb{w}_{\alpha_{2}} \mathbb{w}_{\alpha_{1}} \in W$ in a minimal decomposition, we let

$$
\bar{w}:=\bar{w}_{\alpha_{k}} \ldots \bar{w}_{\alpha_{2}} \bar{w}_{\alpha_{1}} \in W^{\prime}
$$

be its representative in $W^{\prime}$, which is independent of the minimal decomposition (see [Ste16, Lemma 83 (b)]). In particular, we denote by $\bar{w}_{G} \in \bar{G}$ the above representative of the longest Weyl element $\mathbb{w}_{G}$. Note that we also have the natural representative

$$
w:=w_{\alpha_{k}} \ldots w_{\alpha_{2}} w_{\alpha_{1}} \in G
$$

of w. In particular, one has the representative $w_{G} \in G$ for $\mathbb{w}_{G}$, which is the image of $\bar{w}_{G}$ in $G$.

2.2. Dual group and $L$-group. For a cover $(n, \overline{\mathbf{G}})$ associated to $(D, \eta)$, with $Q$ and $B_{Q}$ arising from $D$, we define

$$
Y_{Q, n}:=Y \cap n Y^{*},
$$

where $Y^{*} \subset Y \otimes \mathbf{Q}$ is the dual lattice of $Y$ with respect to $B_{Q}$; more explicitly,

$$
Y_{Q, n}=\left\{y \in Y: B_{Q}\left(y, y^{\prime}\right) \in n \mathbf{Z} \text { for all } y^{\prime} \in Y\right\} \subset Y .
$$

For every $\alpha^{\vee} \in \Phi^{\vee}$, denote

$$
n_{\alpha}:=\frac{n}{\operatorname{gcd}\left(n, Q\left(\alpha^{\vee}\right)\right)}
$$

and

$$
\alpha_{Q, n}^{\vee}=n_{\alpha} \alpha^{\vee}, \quad \alpha_{Q, n}=\frac{\alpha}{n_{\alpha}}
$$

Let

$$
Y_{Q, n}^{s c} \subset Y_{Q, n}
$$

be the sublattice generated by $\Phi_{Q, n}^{\vee}=\left\{\alpha_{Q, n}^{\vee}: \alpha^{\vee} \in \Phi^{\vee}\right\}$. Denote $X_{Q, n}=\operatorname{Hom}_{\mathbf{Z}}\left(Y_{Q, n}, \mathbf{Z}\right)$ and $\Phi_{Q, n}=\left\{\alpha_{Q, n}: \alpha \in \Phi\right\}$. We also write

$$
\Delta_{Q, n}^{\vee}=\left\{\alpha_{Q, n}^{\vee}: \alpha^{\vee} \in \Delta^{\vee}\right\} \text { and } \Delta_{Q, n}=\left\{\alpha_{Q, n}: \alpha \in \Delta\right\} .
$$

Then

$$
\left(Y_{Q, n}, \Phi_{Q, n}^{\vee}, \Delta_{Q, n}^{\vee} ; X_{Q, n}, \Phi_{Q, n}^{\vee}, \Delta_{Q, n}\right)
$$


forms a root datum with a choice of simple roots $\Delta_{Q, n}$. It gives a unique (up to unique isomorphism) pinned reductive group $\overline{\mathbf{G}}_{Q, n}^{\vee}$ over $\mathbf{Z}$, called the dual group of $(n, \overline{\mathbf{G}})$. In particular, $Y_{Q, n}$ is the character lattice for $\bar{G}_{Q, n}^{\vee}$ and $\Delta_{Q, n}^{\vee}$ the set of simple roots. Let

$$
\bar{G}_{Q, n}^{\vee}:=\overline{\mathbf{G}}_{Q, n}^{\vee}(\mathbf{C})
$$

be the associated complex dual group. For simplicity, we may also write $\bar{G}^{\vee}$ for $\bar{G}_{Q, n}^{\vee}$. One has

$$
Z\left(\bar{G}_{Q, n}^{\vee}\right)=\operatorname{Hom}\left(Y / Y_{Q, n}, \mathbf{C}\right) .
$$

In [Wei14, Wei18], Weissman constructed the global $L$-group as well as the local $L$ group extension

$$
\bar{G}_{Q, n}^{\vee} \longleftrightarrow{ }^{L} \bar{G} \longrightarrow W_{F},
$$

which is compatible with the global $L$-group extension. (It may as well be an extension over the Weil-Deligne group. However, the Weil group $W_{F}$ suffices in this paper, since we eventually only consider unitary principal series.) His construction of $L$-group is functorial, and in particular it behaves well with respect to the restriction of $\overline{\mathbf{G}}$ to parabolic subgroups. More precisely, let $\mathbf{M} \subset \mathbf{G}$ be a Levi subgroup. By restriction, one has the $n$-cover $\bar{M}$ of $M$. The $L$-groups ${ }^{L} \bar{M}$ and ${ }^{L} \bar{G}$ are compatible, i.e., there are natural homomorphisms of extensions:

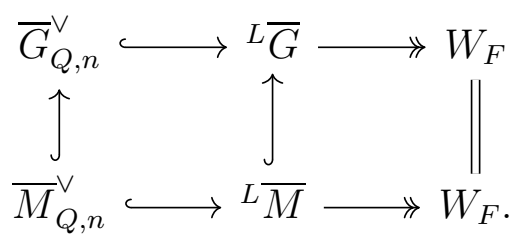

This applies in particular to the case when $M=T$ is a torus.

The extension ${ }^{L} \bar{G}$ does not split over $W_{F}$. However, if $\bar{G}_{Q, n}^{\vee}$ is of adjoint type, then we have a canonical isomorphism

$$
{ }^{L} \bar{G} \simeq \bar{G}_{Q, n}^{\vee} \times W_{F} .
$$

For general $\bar{G}$, under the assumption that $\eta_{n}=\mathbb{1}$, there exists a so-called distinguished genuine character $\chi_{\psi}: Z(\bar{T}) \rightarrow \mathbf{C}^{\times}$(see $[G G 18, \S 6.4]$ ), depending on a nontrivial additive character $\psi$ of $F$, such that $\chi_{\psi}$ gives rise to a splitting of ${ }^{L} \bar{G}$ over $W_{F}$, with respect to which one has an isomorphism

$$
{ }^{L} \bar{G} \simeq_{\chi_{\psi}} \bar{G}_{Q, n}^{\vee} \times W_{F} .
$$

For details on the construction and properties regarding the $L$-group, we refer the reader to [Wei14, Wei18, GG18].

2.3. Twisted Weyl action. For a group $H$ acting on a set $S$, we denote by

$$
\mathcal{O}_{S}^{H}
$$

the set of all $H$-orbits in $S$. For every $z \in S$, denote by $\mathcal{O}_{z}^{H} \in \mathcal{O}_{S}^{H}$ the $H$-orbit of $z$.

Denote by $\mathbb{w}(y)$ the natural Weyl group action on $Y$ and $Y \otimes \mathbf{Q}$ generated by the reflections $\mathbb{w}_{\alpha}$. The two lattices $Y_{Q, n}$ and $Y_{Q, n}^{s c}$ are both $W$-stable under this usual action. Let

$$
\rho:=\frac{1}{2} \sum_{\alpha^{\vee}>0} \alpha^{\vee}
$$

be the half sum of all positive coroots of $\mathbf{G}$. We consider the twisted Weyl-action

$$
\mathbb{w}[y]:=\mathbb{w}(y-\rho)+\rho .
$$


It induces a well-defined twisted action of $W$ on

$$
\mathscr{X}_{Q, n}:=Y / Y_{Q, n}
$$

given by $\mathbb{w}\left[y+Y_{Q, n}\right]:=\mathbb{w}[y]+Y_{Q, n}$, since $W\left(Y_{Q, n}\right)=Y_{Q, n}$ as mentioned above. Thus, we have a permutation representation

$$
\sigma^{\mathscr{X}}: W \longrightarrow \operatorname{Perm}\left(\mathscr{X}_{Q, n}\right),
$$

which plays a pivotal role in the conjectural formulas on Whittaker space, in both [Gaob] and this paper.

We note that the twisted Weyl-action on $Y / Y_{Q, n}^{s c}$ is also well-defined. For every $\alpha \in \Delta$, let $W_{\alpha}=\left\{1, \mathbb{w}_{\alpha}\right\} \subset W$. Arising from the surjection

$$
Y / Y_{Q, n}^{s c} \rightarrow \mathscr{X}_{Q, n}
$$

one has a map of sets

$$
\phi_{\alpha}:\left(Y / Y_{Q, n}^{s c}\right)^{W_{\alpha}} \rightarrow\left(\mathscr{X}_{Q, n}\right)^{W_{\alpha}} .
$$

Recall the following definition from [GSS, Gaob].

Definition 2.1. A covering group $\bar{G}$ of a connected linear reductive group $G$ is called saturated if $Y_{Q, n}^{s c}=Y_{Q, n} \cap Y^{s c}$. It is called of metaplectic type if there exists $\alpha \in \Delta$ such that $\phi_{\alpha}$ is not surjective.

If $G$ is semisimple simply-connected, then $\bar{G}$ is saturated if and only if $\bar{G}^{\vee}$ is of adjoint type. On the other hand, covering groups of metaplectic type are rare. Indeed, it follows from [GSS, $\$ 4.5$ ] that if $G$ is almost simple, then $\bar{G}$ is of metaplectic type if and only if $\mathbf{G}=\mathrm{Sp}_{2 r}$ and $n_{\alpha} \equiv 2(\bmod 4)$ for the unique short simple coroot $\alpha^{\vee}$ of $\mathrm{Sp}_{2 r}$. In particular, the classical double cover of $\mathrm{Sp}_{2 r}$ is such an example.

Throughout the paper, we denote

$$
y_{\rho}:=y-\rho \in Y \otimes \mathbf{Q}
$$

for $y \in Y$. Clearly,

$$
\mathbb{w}[y]-y=\mathbb{w}\left(y_{\rho}\right)-y_{\rho} .
$$

By Weyl action or Weyl orbits in $Y$ or $Y \otimes \mathbf{Q}$ we always refer to the ones with respect to the action $\mathbb{w}[y]$, unless specified otherwise. For simplicity, we will abuse notation and denote by $y$ for an element in $\mathscr{X}_{Q, n}$. We will also write $\mathcal{O}_{\mathscr{X}}$ for the set of $W$-orbits in $\mathscr{X}_{Q, n}$, and use the notation $\mathcal{O}_{z}:=\mathcal{O}_{z}^{W}$, whenever we consider $W$-orbits with respect to the twisted action.

\section{UNITARY UNRAMIFIED PRINCIPAL SERIES}

Henceforth, we assume that $|n|_{F}=1$. Let $K \subset G$ be the hyperspecial maximal compact subgroup generated by $\mathbf{T}(O)$ and $e_{\alpha}(O)$ for all roots $\alpha$. With our assumption that $\eta_{n}$ is trivial, the group $\bar{G}$ splits over $K$ (see [GG18, Theorem 4.2]); we fix such a splitting $s_{K}$. If no confusion arises, we will omit $s_{K}$ and write $K \subset \bar{G}$ instead. Call $\bar{G}$ an unramified covering group in this setting.

A genuine representation $\left(\pi, V_{\pi}\right)$ is called unramified if $\operatorname{dim} V_{\pi}^{K} \neq 0$. Since $\bar{G}$ also splits uniquely over the unipotent subgroup $e_{\alpha}(O)$, we see that $\bar{h}_{\alpha}(u) \in s_{K}(K) \subset \bar{G}$ for every $u \in O^{\times}$. 
3.1. Principal series representation. As $\bar{G}$ splits canonically over the unipotent radical $U$ of the Borel subgroup $B$, we have $\bar{B}=\bar{T} U$. The covering torus $\bar{T}$ is a Heisenberg group. The center $Z(\bar{T})$ of the covering torus $\bar{T}$ is equal to $\phi^{-1}\left(\operatorname{Im}\left(i_{Q, n}\right)\right)$ (see [Wei09]), where

$$
i_{Q, n}: Y_{Q, n} \otimes F^{\times} \rightarrow T
$$

is the isogeny induced from the embedding $Y_{Q, n} \subset Y$.

Let $\chi \in \operatorname{Hom}_{\epsilon}\left(Z(\bar{T}), \mathbf{C}^{\times}\right)$be a genuine character of $Z(\bar{T})$, write

$$
i(\chi):=\operatorname{Ind} \frac{\bar{T}}{A} \chi^{\prime}
$$

for the induced representation of $\bar{T}$, where $\bar{A}$ is a maximal abelian subgroup of $\bar{T}$, and $\chi^{\prime}$ is an extension of $\chi$ to $\bar{A}$. By the Stone-von Neumann theorem (see [Wei09, Theorem 3.1]), the construction

$$
\chi \mapsto i(\chi)
$$

gives a bijection between the isomorphism classes of genuine representations of $Z(\bar{T})$ and $\bar{T}$. Since we consider unramified covering group $\bar{G}$ in this paper, we take

$$
\bar{A}:=Z(\bar{T}) \cdot(K \cap T) \text {. }
$$

The left action of $w$ on $i(\chi)$ is given by ${ }^{w} i(\chi)(\bar{t})=i(\chi)\left(w^{-1} \bar{t} w\right)$. The group $W$ does not act on $i(\chi)$, but only on its isomorphism classes. On the other hand, we have a well-defined action of $W$ on $\chi$ :

$$
\left({ }^{w} \chi\right)(\bar{t}):=\chi\left(w^{-1} \bar{t} w\right) .
$$

Viewing $i(\chi)$ as a genuine representation of $\bar{B}$ by inflation from the quotient map $\bar{B} \rightarrow \bar{T}$, we denote by

$$
I(i(\chi)):=\operatorname{Ind} \frac{\bar{G}}{B} i(\chi)
$$

the normalized induced principal series representation of $\bar{G}$. For simplicity, we may also write $I(\chi)$ for $I(i(\chi))$. One knows that $I(\chi)$ is unramified (i.e., $I(\chi)^{K} \neq 0$ ) if and only if $\chi$ is unramified, i.e., $\chi$ is trivial on $Z(\bar{T}) \cap K$. Moreover, the Satake isomorphism for $\bar{G}$ implies that a genuine representation is unramified if and only if it is a subquotient of an unramified principal series.

Setting $\bar{Y}_{Q, n}:=Z(\bar{T}) /(Z(\bar{T}) \cap K)$, one has a natural abelian extension

$$
u_{n} \longleftrightarrow \bar{Y}_{Q, n} \stackrel{\varphi}{\longrightarrow} Y_{Q, n}
$$

such that unramified genuine characters of $Z(\bar{T})$ correspond to genuine characters of $\bar{Y}_{Q, n}$. Since $\bar{A} /(T \cap K) \simeq \bar{Y}_{Q, n}$ as well, there is a canonical extension (also denoted by $\chi)$ of an $\chi$ to $\bar{A}$, by composing it with $\bar{A} \rightarrow \bar{Y}_{Q, n}$. Therefore, we will identify $i(\chi)$ with $\operatorname{Ind} \frac{\bar{T}}{A} \chi$ for this canonical extension $\chi$.

3.2. $\gamma$-function. Let $\chi: F^{\times} \rightarrow \mathbf{C}^{\times}$be a linear character. Tate [Tat67] defined a gamma factor $\gamma(s, \underline{\chi}, \psi), s \in \overline{\mathbf{C}}$ which is essentially the ratio of two integrals of a test function and its Fourier transform (depending on a nontrivial character $\psi$ ). We have

$$
\gamma(s, \underline{\chi}, \psi)=\varepsilon(s, \underline{\chi}, \psi) \cdot \frac{L\left(1-s, \underline{\chi}^{-1}\right)}{L(s, \underline{\chi})},
$$

where $L(s, \underline{\chi})$ is the $L$-function of $\underline{\chi}$. If $\underline{\chi}$ is unramified and the conductor of $\psi$ is $O$, then

$$
\varepsilon(s, \underline{\chi}, \psi)=1 \text { and } L(s, \underline{\chi})=\left(1-q^{-s} \underline{\chi}(\varpi)\right)^{-1} .
$$


In this case, we write

$$
\gamma(s, \underline{\chi}):=\gamma(s, \underline{\chi}, \psi)=\frac{1-q^{-s} \underline{\chi}(\varpi)}{1-q^{-1+s} \underline{\chi}(\varpi)^{-1}}
$$

with the $\psi$ omitted.

Let $\chi$ be a genuine unramified character of $Z(\bar{T})$. For every $\alpha \in \Phi$,

$$
\underline{\chi}_{\alpha}: F^{\times} \rightarrow \mathbf{C}^{\times} \text {given by } \underline{\chi}_{\alpha}(a):=\chi\left(\bar{h}_{\alpha}\left(a^{n_{\alpha}}\right)\right)
$$

is an unramified character of $F^{\times}$. We also use in this paper the shorthand notation

$$
\chi_{\alpha}:=\underline{\chi}_{\alpha}(\varpi) \in \mathbf{C}^{\times}
$$

for every root $\alpha$. For $\mathbb{w}=\mathbb{w}_{\alpha}$, we define the gamma factor $\gamma\left(w_{\alpha}, \chi\right)$ to be such that

$$
\gamma\left(w_{\alpha}, \chi\right)^{-1}=\gamma\left(0, \underline{\chi}_{\alpha}\right)^{-1}=\frac{1-q^{-1} \chi\left(\bar{h}_{\alpha}\left(\varpi^{n_{\alpha}}\right)\right)^{-1}}{1-\chi\left(\bar{h}_{\alpha}\left(\varpi^{n_{\alpha}}\right)\right)} .
$$

For general $w \in W$, define

$$
\gamma(w, \chi)=\prod_{\alpha \in \Phi_{\mathrm{w}}} \gamma\left(w_{\alpha}, \gamma\right)
$$

where $\Phi_{\mathbb{w}}=\{\alpha>0: \mathbb{w}(\alpha)<0\}$.

3.3. Intertwining operator. For $w \in W$, let $A(w, \chi): I(\chi) \rightarrow I\left({ }^{w} \chi\right)$ be the intertwining operator defined by

$$
A(w, \chi)(f)(\bar{g})=\int_{U_{w}} f\left(\bar{w}^{-1} u \bar{g}\right) d u
$$

whenever it is absolutely convergent. It can be meromorphically continued for all $\chi$ (see $[\mathrm{McN1} 12, \S 7])$. The operator $A(w, \chi)$ satisfies the cocycle relation as in the linear case. Let $f_{0} \in I(\chi)$ and $f_{0}^{\prime} \in I\left({ }^{w} \chi\right)$ be the normalized unramified vectors. We have

$$
A(w, \chi)\left(f_{0}\right)=c_{\mathrm{gk}}(w, \chi) f_{0}^{\prime}
$$

where

$$
c_{\mathrm{gk}}(w, \chi)=\prod_{\alpha \in \Phi_{\mathrm{w}}} \frac{1-q^{-1} \chi\left(\bar{h}_{\alpha}\left(\varpi^{n_{\alpha}}\right)\right)}{1-\chi\left(\bar{h}_{\alpha}\left(\varpi^{n_{\alpha}}\right)\right)} .
$$

The Plancherel measure $\mu(w, \chi)$ associated with $A(w, \chi)$ is such that

$$
A\left(w^{-1},{ }^{w} \chi\right) \circ A(w, \chi)=\mu(w, \chi)^{-1} \cdot \mathrm{id} ;
$$

more explicitly,

$$
\mu(w, \chi)^{-1}=c_{\mathrm{gk}}\left(w^{-1},{ }^{w} \chi\right) \cdot c_{\mathrm{gk}}(w, \chi) .
$$

To recall the interpretation of $c_{\mathrm{gk}}(w, \chi)$ in terms of $L$-functions, we first recall the set-up on the dual side. Consider the adjoint representation

$$
A d_{\overline{\mathfrak{u}}^{\vee}}:{ }^{L} \bar{T} \rightarrow \mathrm{GL}\left(\overline{\mathfrak{u}}^{\vee}\right),
$$

where $\overline{\mathfrak{u}}^{\vee}$ is the Lie algebra of unipotent radical $\bar{U}^{\vee}$ of the Borel subgroup $\bar{T}^{\vee} \bar{U}^{\vee} \subset \bar{G}^{\vee}$. It factors through $A d_{\overline{\mathfrak{u}}^{\vee}}^{\mathbf{C}}$ :

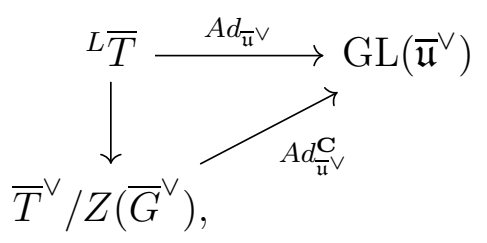

as $Z\left(\bar{G}^{\vee}\right)$ acts trivially on $\overline{\mathfrak{u}}^{\vee}$. 
Therefore, irreducible subspaces of $\overline{\mathfrak{u}}^{\vee}$ for $A d_{\overline{\mathfrak{u}}^{\vee}}$ are in one-to-one correspondence with irreducible subspaces with respect to $A d_{\overline{\mathrm{u}}}^{\mathbf{C}}$, which are just the one-dimensional spaces associated to the positive roots of $\bar{G}^{\vee}$. More precisely, we have the decomposition of $A d_{\overline{\mathrm{u}} \vee}$ into irreducible ${ }^{L} \bar{T}$-modules:

$$
A d_{\bar{u}^{\vee}}=\bigoplus_{\alpha>0} A d_{\alpha}
$$

where $\left(A d_{\alpha}, V_{\alpha}\right) \subseteq \overline{\mathfrak{u}}^{\vee}$ is spanned by a basis vector $E_{\alpha_{Q, n}^{\vee}}$ in the Lie algebra $\overline{\mathfrak{u}}_{\alpha}^{\vee}$ associated to the positive root $\alpha_{Q, n}^{\vee}$ of $\bar{G}^{\vee}$.

By the local Langlands correspondence for covering torus (see [Wei18, §10] or [GG18, $\S 8])$, associated to $i(\chi)$ we have a splitting

$$
\phi_{\chi}: W_{F} \longrightarrow{ }^{L} \bar{T}
$$

of the L-group extension

$$
\bar{T}^{\vee} \longleftrightarrow{ }^{L} \bar{T} \longrightarrow W_{F}
$$

where $\bar{T}^{\vee}=\operatorname{Hom}\left(Y_{Q, n}, \mathbf{C}^{\times}\right)$is the dual group of $\bar{T}$. Then $L\left(s, i(\chi), A d_{\alpha}\right)$ is by definition equal to the local Artin $L$-function $L\left(s, A d_{\alpha} \circ \phi_{\chi}\right)$ associated with $A d_{\alpha} \circ \phi_{\chi}$, i.e.,

$$
L\left(s, i(\chi), A d_{\alpha}\right):=L\left(s, A d_{\alpha} \circ \phi_{\chi}\right)=\operatorname{det}\left(1-\left.q^{-s} A d_{\alpha} \circ \phi_{\chi}(\text { Frob })\right|_{V_{\alpha}^{I}}\right)^{-1} .
$$

For unramified $i(\chi)$ (equivalently, unramified $\chi$ ), the inertia group $I$ acts trivially on $V_{\alpha}$. It follows that

$$
L\left(s, i(\chi), A d_{\alpha}\right)=\operatorname{det}\left(1-\left.q^{-s} A d_{\alpha} \circ \phi_{\chi}(\varpi)\right|_{V_{\alpha}}\right)^{-1} .
$$

Moreover, by [Gao18, Theorem 7.8], one has

$$
\phi_{\chi} \circ A d(\varpi)\left(E_{\alpha_{Q, n}^{\vee}}\right)=\chi\left(\bar{h}_{\alpha}\left(\varpi^{n_{\alpha}}\right)\right) \cdot E_{\alpha_{Q, n}^{\vee}}
$$

and therefore

$$
L\left(s, i(\chi), A d_{\alpha}\right)=\left(1-q^{-s} \cdot \chi\left(\bar{h}_{\alpha}\left(\varpi^{n_{\alpha}}\right)\right)\right)^{-1}=L\left(s, \underline{\chi}_{\alpha}\right) .
$$

Moreover, if we denote

$$
E_{\mathrm{w}}:=\bigoplus_{\mathrm{w} \in \Phi_{\mathrm{w}}} \mathbf{C} \cdot E_{\alpha_{Q, n}^{\vee}}
$$

and let $A d_{\mathrm{w}}$ be the restriction of the adjoint representation $A d$ to $E_{\mathrm{w}}$, then the Artin $L$-function associated to $A d_{\mathrm{w}}$ is

$$
L\left(s, A d_{\mathrm{w}} \circ \phi_{\chi}\right)=\prod_{\alpha \in \Phi_{\mathrm{w}}} L\left(s, i(\chi), A d_{\alpha}\right) .
$$

We also denote $L\left(s, i(\chi), A d_{\mathrm{w}}\right):=L\left(s, A d_{\mathrm{w}} \circ \phi_{\chi}\right)$. Thus,

$$
c_{\mathrm{gk}}(w, \chi)=\frac{L\left(0, i(\chi), A d_{\mathrm{w}}\right)}{L\left(1, i(\chi), A d_{\mathrm{w}}\right)} .
$$

3.4. Normalization. For $\mathbb{w} \in W$, we normalize the intertwining operator by

$$
\mathscr{A}(w, \chi):=c_{\mathrm{gk}}(w, \chi)^{-1} \cdot A(w, \chi)=\frac{L\left(1, i(\chi), A d_{\mathrm{w}}\right)}{L\left(0, i(\chi), A d_{\mathrm{w}}\right)} \cdot A(w, \chi) .
$$

Note that in our setting $\varepsilon\left(s, \underline{\chi}_{\alpha}, \psi\right)=1$ for every $\alpha$, and thus the above normalization is the same as the one for linear algebraic groups, first proposed by Langlands [Lan76] and investigated in [KS88, Sha90] for example. 
Proposition 3.1. Let $\chi$ be an unramified genuine character of $Z(\bar{T})$. For every $\mathbb{w}_{1}, \mathbb{w}_{2} \in$ $W$ (with no requirement on the length),

$$
\mathscr{A}\left(w_{2} w_{1}, \chi\right)=\mathscr{A}\left(w_{2},{ }^{w_{1}} \chi\right) \circ \mathscr{A}\left(w_{1}, \chi\right) \text {. }
$$

In particular, $\mathscr{A}\left(w^{-1},{ }^{w} \chi\right) \circ \mathscr{A}(w, \chi)=\mathrm{id}$ for every $\mathbb{w} \in W$. Moreover, if $\chi$ is also unitary, then $\mathscr{A}(w, \chi)$ is holomorphic.

Proof. The proof is essentially that of Winarsky [Win78, Page 951-952], which relies on an inductive argument on the length of $\mathrm{w}_{2}$ and also the basic step

$$
\mathscr{A}\left(w_{\alpha},{ }^{w_{\alpha}} \chi\right) \circ \mathscr{A}\left(w_{\alpha}, \chi\right)=\mathrm{id}
$$

However, this last equality follows from (11) and the normalization of $\mathscr{A}\left(w_{\alpha}, \chi\right)$ in (14). See [McN12, Page 313-314] for some details of the argument in the context of covering groups.

\section{R-GROUP}

From now on, we assume that $\chi$ is a unitary unramified genuine character of $Z(\bar{T})$. Let

$$
W_{\chi}:=\left\{\mathbb{w} \in W:{ }^{w} \chi=\chi\right\} \subset W .
$$

Proposition 3.1 shows that

$$
\mathbb{w} \mapsto \mathscr{A}(w, \chi)
$$

gives rise to a group homomorphism

$$
\tau_{\chi}: W_{\chi} \longrightarrow \operatorname{Isom}_{\bar{G}}(I(\chi)),
$$

where $\operatorname{Isom}_{\bar{G}}(I(\chi))$ denotes the group of $\bar{G}$-isomorphisms of $I(\chi)$. Let $\operatorname{End}_{\bar{G}}(I(\chi))$ be the commuting algebra of $I(\chi)$. The group homomorphism $\tau_{\chi}$ gives an algebra homomorphism which, by abuse of notation, is also denoted by

$$
\tau_{\chi}: \mathbf{C}\left[W_{\chi}\right] \longrightarrow \operatorname{End}_{\bar{G}}(I(\chi)) .
$$

However, $\tau_{\chi}$ is not an isomorphism in general.

We would like to define a subgroup $R_{\chi} \subset W_{\chi}$ such that $\tau_{\chi}$ induces an algebra isomorphism

$$
\mathbf{C}\left[R_{\chi}\right] \simeq \operatorname{End}_{\bar{G}}(I(\chi)) \text {. }
$$

For this purpose, consider the set

$$
\Phi_{\chi}=\left\{\alpha>0: \underline{\chi}_{\alpha}=\mathbb{1}\right\} \subset \Phi,
$$

where $\underline{\chi}_{\alpha}$ is as in (10). Let $W_{\chi}^{0} \subset W$ be the subgroup generated by $\left\{\mathbb{w}_{\alpha}: \alpha \in \Phi_{\chi}\right\}$. It follows from [Gaob, Lemma 3.1] that $\mathrm{w}_{\alpha} \in W_{\chi}$ if $\alpha \in \Phi_{\chi}$. Therefore,

$$
W_{\chi}^{0} \subseteq W_{\chi}
$$

and we have a short exact sequence:

$$
W_{\chi}^{0} \longrightarrow W_{\chi} \longrightarrow R_{\chi},
$$

where $R_{\chi}:=W_{\chi} / W_{\chi}^{0}$. The sequence splits with a natural splitting $s: R_{\chi} \hookrightarrow W_{\chi}$ given as follows. Consider the group

$$
W\left(\Phi_{\chi}\right)=\left\{\mathbb{w} \in W: \mathbb{w}\left(\Phi_{\chi}\right)=\Phi_{\chi}\right\} .
$$

Then one has

$$
R_{\chi} \simeq W_{\chi} \cap W\left(\Phi_{\chi}\right)
$$


or more explicitly,

$$
\begin{aligned}
R_{\chi} & \simeq\left\{\mathbb{w} \in W_{\chi}: \mathbb{w}\left(\Phi_{\chi}\right)=\Phi_{\chi}\right\} \\
& =\left\{\mathbb{w} \in W_{\chi}: \alpha>0 \text { and } \alpha \in \Phi_{\chi} \text { imply that } \mathbb{w}(\alpha)>0\right\} \\
& =\left\{\mathbb{w} \in W_{\chi}: \Phi_{\mathbb{w}} \cap \Phi_{\chi}=\emptyset\right\} .
\end{aligned}
$$

This gives that $W_{\chi} \simeq W_{\chi}^{0} \rtimes R_{\chi}$. We always identity $R_{\chi}$ with $W_{\chi} \cap W\left(\Phi_{\chi}\right)$.

Before we proceed, we first show that for $w \in W_{\chi}$, the two factors $c_{\mathrm{gk}}(w, \chi)$ and $\gamma(w, \chi)^{-1}$ are actually equal.

Lemma 4.1. Let $\bar{G}$ be an $n$-fold cover of a connected reductive group $G$, and $\chi$ a unitary unramified character of $Z(\bar{T})$. For every $\mathbb{w} \in W_{\chi}$, one has

$$
L\left(s, A d_{\mathrm{w}} \circ \phi_{\chi}\right)=L\left(s, A d_{\mathrm{w}}^{\vee} \circ \phi_{\chi}\right),
$$

where $A d_{\mathrm{w}}^{\vee}$ is the contragredient representation of $A d_{\mathrm{w}}$. Therefore, for every $\mathbb{w} \in W_{\chi}$,

$$
c_{\mathrm{gk}}(w, \chi)^{-1}=\gamma(w, \chi),
$$

which is nonzero if $\mathrm{w} \in R_{\chi}$.

Proof. The argument is already in [KS88, Lemma 4.2]. First, since $\mathbb{w} \in W_{\chi}$, the two representations $A d_{\mathrm{w}} \circ \phi_{\chi}$ and $A d_{\mathrm{w}}^{\vee} \circ \phi_{\chi}$ are equivalent. Therefore, $L\left(s, A d_{\mathrm{w}} \circ \phi_{\chi}\right)=$ $L\left(s, A d_{\mathrm{w}}^{\vee} \circ \phi_{\chi}\right)$. Now for $\mathrm{w} \in W_{\chi}$, we have

$$
c_{\mathrm{gk}}(w, \chi)^{-1}=\frac{L\left(1, A d_{\mathrm{w}} \circ \phi_{\chi}\right)}{L\left(0, A d_{\mathrm{w}} \circ \phi_{\chi}\right)}=\frac{L\left(1, A d_{\mathrm{w}}^{\vee} \circ \phi_{\chi}\right)}{L\left(0, A d_{\mathrm{w}} \circ \phi_{\chi}\right)}=\gamma(w, \chi) .
$$

The proof is completed in view of the fact that $1-\underline{\chi}_{\alpha}(\varpi) \neq 0$ for every $\alpha \in \Phi_{\mathrm{w}}$, if $\mathbb{w} \in R_{\chi}$.

The main theorem on $R$-group is as follows:

Theorem 4.2 ([KS75, Sil78, Li14, Li12, Luo]). For a unitary unramified genuine character $\chi$ of $Z(\bar{T})$, one has

$$
W_{\chi}^{0}=\{w: \mathscr{A}(w, \chi) \text { is a scalar }\} .
$$

Moreover, the algebra $\operatorname{End}_{\bar{G}}(I(\chi))$ has a basis given by $\left\{\mathscr{A}(w, \chi): \mathbb{w} \in R_{\chi}\right\}$, and by restriction $\tau_{\chi}$ gives a natural algebra isomorphism

$$
\mathbf{C}\left[R_{\chi}\right] \simeq \operatorname{End}_{\bar{G}}(I(\chi)) .
$$

For a general parabolic induction of linear algebraic groups, the analogous result was first shown by Knapp-Stein [KS75] for real groups, and by Silberger [Sil78] for $p$-adic groups. The generalization to covering groups includes the work of W.-W. Li [Li14,Li12], which shows that Harish-Chandra's harmonic analysis extends to the covering setting. In particular, the Harish-Chandra $c$-function and Plancherel measure are discussed in detail in loc. cit.. Finally, in the recent work of C.-H. Luo [Luo], the Harish-Chandra commuting algebra theorem is proved for general parabolic induction on covering groups, and in particular in the minimal parabolic case the isomorphism $\mathbf{C}\left[R_{\chi}\right] \simeq \operatorname{End}_{\bar{G}}(I(\chi))$ is established.

Let

$$
I(\chi)=\bigoplus_{i=1}^{k} m_{i} \pi_{i}
$$

be the decomposition of $I(\chi)$ into irreducible subrepresentations with multiplicities $m_{i}$. Denote

$$
\Pi(\chi):=\left\{\pi_{i}: 1 \leq i \leq k\right\}
$$


which constitutes the $L$-packet associated to the parameter $\phi_{\chi}$ corresponding to $I(\chi)$. Some immediate consequences of Theorem 4.2 are:

(i) $\operatorname{dim} \operatorname{End}_{\bar{G}}(I(\chi))=\left|R_{\chi}\right|$ and $\mathbf{C}\left[R_{\chi}\right] \simeq \bigoplus_{i=1}^{k} M_{m_{i}}(\mathbf{C})$.

(ii) $m_{i}=1$ for all $i$ if and only if $R_{\chi}$ is abelian.

(iii) in general, $k$ is equal to the dimension of the center of $\mathbf{C}\left[R_{\chi}\right]$, which is equal to the the number of conjugacy classes of $R_{\chi}$. Thus, there is a bijective correspondence

$$
\operatorname{Irr}\left(R_{\chi}\right) \longleftrightarrow \Pi(\chi) \text {. }
$$

Since we are dealing with unramified $\chi$, we will show later (see Theorem 4.6) that $R_{\chi}$ is actually abelian, and thus $I(\chi)$ is multiplicity-free.

\subsection{Parametrization of $\Pi(\chi)$. Denote the above correspondence}

$$
\operatorname{Irr}\left(R_{\chi}\right) \longleftrightarrow \Pi(\chi)
$$

from (iii) by

$$
\sigma \leftrightarrow \pi_{\sigma} \text {, and } \sigma_{\pi} \leftrightarrow \pi
$$

The correspondence is not canonical, and can be twisted by characters of $R_{\chi}$. However, in the unramified setting, we have a natural parametrization given as follows (see [Key87, Page 39]).

Denote by $\theta_{\sigma}$ the character of $\sigma \in \operatorname{Irr}\left(R_{\chi}\right)$. Consider the operator

$$
P_{\sigma}:=\frac{\operatorname{dim} \sigma}{\left|R_{\chi}\right|} \sum_{\mathrm{w} \in R_{\chi}} \overline{\theta_{\sigma}(\mathbb{w})} \cdot \mathscr{A}(w, \chi) .
$$

Since the characters $\left\{\theta_{\sigma}: \sigma \in \operatorname{Irr}\left(R_{\chi}\right)\right\}$ are orthogonal, the operators $P_{\sigma}$ are orthogonal projections of $I(\chi)$ onto nonzero invariant subspaces. Denote

$$
V(\sigma):=P_{\sigma}(I(\chi)) .
$$

Then $V(\sigma)$ 's are pairewise disjoint and we have

$$
I(\chi)=\bigoplus_{\sigma \in \operatorname{Irr}\left(R_{\chi}\right)} V(\sigma)
$$

Since the number of inequivalent constituents of $I(\chi)$ is equal to $\left|\operatorname{Irr}\left(R_{\chi}\right)\right|$, it follows that $V(\sigma)$ is an isotypic sum of an irreducible representation. Thus, we write

$$
V(\sigma)=m_{\pi_{\sigma}} \cdot \pi_{\sigma},
$$

and this gives a correspondence $\sigma \mapsto \pi_{\sigma}$. We also have

$$
m_{\pi_{\sigma}}=\operatorname{dim} \sigma \text {. }
$$

The inverse $\pi \mapsto \sigma_{\pi}$ can be described explicitly as well, see [Key87, Page 39]. The group $\operatorname{Hom}\left(R_{\chi}, \mathbf{C}^{\times}\right)$acts on $\Pi(\chi)$ by transporting the obvious action on $\operatorname{Irr}\left(R_{\chi}\right)$ given by

$$
\xi \otimes \sigma, \text { where } \xi \in \operatorname{Hom}\left(R_{\chi}, \mathbf{C}^{\times}\right) \text {and } \sigma \in \operatorname{Irr}\left(R_{\chi}\right) .
$$

This action is free and transitive on the elements $\pi \in \Pi(\chi)$ which occurs with multiplicity one in $I(\chi)$. Thus the correspondence $\sigma \leftrightarrow \pi_{\sigma}$ is not canonical. However, the above parametrization given by (15) (without any further twisting) is already a natural one in view of the following:

Lemma 4.3. With notations as above, then $P_{\mathbb{1}}(I(\chi))=\pi_{\chi}^{u n}$, i.e., the unramified constituent $\pi_{\chi}^{\text {un }}$ of $I(\chi)$ is parametrized by the trivial representation $\mathbb{1} \in \operatorname{Irr}\left(R_{\chi}\right)$. 
Proof. It suffices to show that $P_{\mathbb{1}}\left(f_{0}\right)=f_{0}$ where $f_{0} \in \pi_{\chi}^{u n} \subset I(\chi)$ is the normalized unramified vector. However, by (15), one has

$$
P_{\mathbb{1}}\left(f_{0}\right)=\frac{1}{\left|R_{\chi}\right|} \sum_{\mathbb{w} \in R_{\chi}} \mathscr{A}(w, \chi)\left(f_{0}\right) .
$$

By the normalization in $\mathscr{A}(w, \chi)$, one has $\mathscr{A}(w, \chi)\left(f_{0}\right)=f_{0}$ for every $\mathbb{w} \in R_{\chi}$ (in fact for every $\left.\mathbb{w} \in W_{\chi}\right)$. Thus, $P_{\mathbb{1}}\left(f_{0}\right)=f_{0}$, and this gives the desired conclusion.

4.2. Some analysis on $R_{\chi}$. From this subsection to $\S 4.4$, we will analyze the group $R_{\chi}$ by relating it to the work [Key82b] of Keys on simply-connected Chevalley groups. More precisely, we will show that there is a group $R_{\chi}^{s c}$ containing $R_{\chi}$ such that:

(i) The quotient $R_{\chi}^{s c} / R_{\chi} \simeq W_{\chi}^{s c} / W_{\chi}$ can be understood from the dual side in terms of the $L$-parameter $\phi_{\chi}$. In particular, the size of $R_{\chi}^{s c} / R_{\chi}$ is related to the center $Z\left(\bar{G}^{\vee}\right)$ of the dual group $\bar{G}^{\vee}$.

(ii) The group $R_{\chi}^{s c}$ is equal to the R-group of a unramified principal series on a simplyconnected Chevalley group, which is determined in [Key82b]. In particular, $R_{\chi}^{s c}$ is abelian, and this enforces $R_{\chi}$ to be abelian. However, since $R_{\chi}$ is not equal to $R_{\chi}^{s c}$ in general, this shows that unitary genuine principal series of a covering group tends to be less reducible than the linear algebraic group.

For this purpose, we first define a group $W_{\chi}^{s c}$ such that

$$
W_{\chi} \subseteq W_{\chi}^{s c} \subseteq W
$$

Let $\mathbf{T}_{Q, n}$ and $\mathbf{T}_{Q, n}^{s c}$ be the split tori defined over $F$ associated to the two lattices $Y_{Q, n}$ and $Y_{Q, n}^{s c}$ respectively. Denote by $T_{Q, n}$ and $T_{Q, n}^{s c}$ their $F$-rational points. Let $T_{Q, n}^{\dagger}$ and $T_{Q, n}^{s c, \dagger}$ be the image of $T_{Q, n}$ and $T_{Q, n}^{s c}$ in $T$ with respect to the two maps

$$
i_{Q, n}: T_{Q, n} \rightarrow T \text { and } i^{s c}: T_{Q, n}^{s c} \rightarrow T,
$$

which are induced from $Y_{Q, n} \hookrightarrow Y$ and $Y_{Q, n}^{s c} \hookrightarrow Y$ respectively. Recalling the projection

$$
\phi: \bar{G} \rightarrow G
$$

we have from $\S 3.1$ that

$$
Z(\bar{T})=\phi^{-1}\left(T_{Q, n}^{\dagger}\right)
$$

We denote

$$
Z(\bar{T})^{s c}:=\phi^{-1}\left(T_{Q, n}^{s c, \dagger}\right) \subset Z(\bar{T})
$$

Let

$$
\chi^{s c}:=\left.\chi\right|_{Z(\bar{T})^{s c}}
$$

be the genuine character of $Z(\bar{T})^{s c}$ obtained from the restriction of $\chi$. Consider

$$
W_{\chi}^{s c}:=\left\{\mathbb{w} \in W:{ }^{w}\left(\chi^{s c}\right)=\chi^{s c}\right\} \supset W_{\chi}
$$

and analogously

$$
R_{\chi}^{s c}:=W_{\chi}^{s c} / W_{\chi}^{0}
$$

which then contains $R_{\chi}$. A splitting $s^{s c}$ of $R_{\chi}^{s c}$ into $W_{\chi}^{s c}$ is given by

$$
R_{\chi}^{s c} \simeq W_{\chi}^{s c} \cap W\left(\Phi_{\chi}\right)
$$


In summary, one has a commutative diagram with exact rows and compatible splittings:

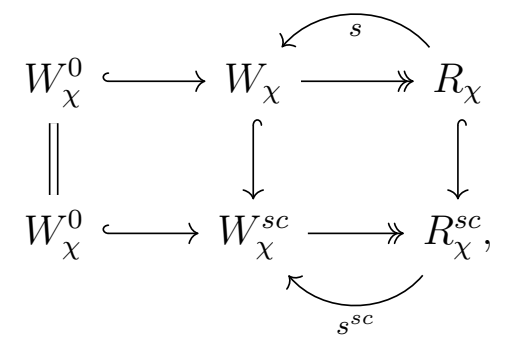

where $s$ and $s^{s c}$ are the aforementioned natural splittings of $R_{\chi}$ and $R_{\chi}^{s c}$ respectively. It follows immediately that one has an isomorphism of finite groups:

$$
R_{\chi}^{s c} / R_{\chi} \simeq W_{\chi}^{s c} / W_{\chi}
$$

4.3. The quotient $R_{\chi}^{s c} / R_{\chi}$. Recall the $L$-parameter

$$
\phi_{\chi}: W_{F} \longrightarrow{ }^{L} \bar{T}
$$

associated to $i(\chi)$, which is a splitting of the extension

$$
\bar{T}^{\vee} \longleftrightarrow{ }^{L} \bar{T} \longrightarrow W_{F} .
$$

In fact $\phi_{\chi}$ only depends on $\chi$, and this justifies our notation. Recall also that $\bar{T}^{\vee}=$ $\operatorname{Hom}\left(Y_{Q, n}, \mathbf{C}^{\times}\right)$is the dual group of $\bar{T}$ and

$$
Z\left(\bar{G}^{\vee}\right) \simeq \operatorname{Hom}\left(Y_{Q, n} / Y_{Q, n}^{s c}, \mathbf{C}^{\times}\right) .
$$

Denote

$$
\bar{T}_{a d}^{\vee}:=\bar{T}^{\vee} / Z\left(\bar{G}^{\vee}\right) \simeq \operatorname{Hom}\left(Y_{Q, n}^{s c}, \mathbf{C}^{\times}\right) .
$$

Pushing out the exact sequence in (16) via the map $f: \bar{T}^{\vee} \rightarrow \bar{T}^{\vee} / Z\left(\bar{G}^{\vee}\right)$, one obtains a short exact sequence

$$
\bar{T}_{a d}^{\vee}{ }^{L} \bar{T}^{s c}:=f_{*}\left({ }^{L} \bar{T}\right) \longrightarrow W_{F} .
$$

Denote by $\operatorname{Spl}\left({ }^{L} \bar{T}, W_{F}\right)$ the set of splittings of (16), and similarly $\operatorname{Spl}\left({ }^{L} \bar{T}^{s c}, W_{F}\right)$. Then $f$ induces a map

$$
f_{*}: \operatorname{Spl}\left({ }^{L} \bar{T}, W_{F}\right) \longrightarrow \operatorname{Spl}\left({ }^{L} \bar{T}^{s c}, W_{F}\right),
$$

which arises from the obvious composite. The Weyl group $W$ acts naturally on the two groups ${ }^{L} \bar{T}$ and ${ }^{L} \bar{T}^{s c}$, and the map $f_{*}$ in (18) is $W$-equivariant. Here $f_{*}\left(\phi_{\chi}\right)$ is naturally associated with $\chi^{s c}$. Since the LLC for covering torus is $W$-equivariant (see [GG18, §9.3]), we have

and

$$
W_{\chi}=\operatorname{Stab}_{W}\left(\phi_{\chi}\right)
$$

$$
W_{\chi}^{s c}=\operatorname{Stab}_{W}\left(f_{*}\left(\phi_{\chi}\right)\right)
$$

Here $\operatorname{Stab}_{W}(x)$ denotes the subgroup of stabilizers of $x$ in $W$.

As it might be more convenient to work with parameters valued in the dual group (instead of the $L$-group), we can have the following reduction. From $\S 2.2$, we have a distinguished genuine character $\chi_{\psi}$ of $Z(\bar{T})$ depending on a nontrivial additive character $\psi$ of $F$. This gives a splitting $\phi_{\chi_{\psi}} \in \operatorname{Spl}\left({ }^{L} \bar{T}, W_{F}\right)$, which is fixed by $W$ since $\chi_{\psi}$ is $W$-invariant (see $[G G 18, \S 6.5]$ ). From this, one obtains a "relative" version of (18):

$$
f_{*}^{\psi}: \operatorname{Hom}\left(W_{F}, \bar{T}^{\vee}\right) \longrightarrow \operatorname{Hom}\left(W_{F}, \bar{T}_{a d}^{\vee}\right) .
$$


If we denote

$$
\phi_{\chi}^{\psi}:=\phi_{\chi} / \phi_{\chi_{\psi}} \in \operatorname{Hom}\left(W_{F}, \bar{T}^{\vee}\right),
$$

then $f_{*}^{\psi}$ sends $\phi_{\chi}^{\psi}$ to

$$
\frac{f_{*}\left(\phi_{\chi}\right)}{f_{*}\left(\phi_{\chi_{\psi}}\right)} \in \operatorname{Hom}\left(W_{F}, \bar{T}_{a d}^{\vee}\right) .
$$

The kernel of $f_{*}^{\psi}$ is given by

$$
\operatorname{Ker}\left(f_{*}^{\psi}\right)=\operatorname{Hom}\left(W_{F}, Z\left(\bar{G}^{\vee}\right)\right) .
$$

Since $f_{*}^{\psi}$ is also $W$-equivariant, we have

$$
W_{\chi}=\operatorname{Stab}_{W}\left(\phi_{\chi}^{\psi}\right), \quad W_{\chi}^{s c}=\operatorname{Stab}_{W}\left(f_{*}^{\psi}\left(\phi_{\chi}^{\psi}\right)\right) .
$$

Assume henceforth that the conductor of $\psi$ is $O$. In this case, $\chi_{\psi}$ is unramified; thus $\phi_{\chi}^{\psi}$ is unramified and $\phi_{\chi}^{\psi}(\varpi) \in \bar{T}^{\vee}$ is the relative Satake parameter for $\chi$. Also, $f_{*}^{\psi}$ is determined by the map

$$
f^{\vee}: \bar{T}^{\vee} \rightarrow \bar{T}_{a d}^{\vee}
$$

such that

$$
f^{\vee}\left(\phi_{\chi}^{\psi}(\varpi)\right)=\left(f_{*}^{\psi}\left(\phi_{\chi}^{\psi}\right)\right)(\varpi) .
$$

For every $t \in \bar{T}^{\vee}$ such that $f^{\vee}(t)$ is fixed by $W_{\chi}^{s c}$, the action of $W_{\chi}^{s c}$ on the set $t \cdot Z\left(\bar{G}^{\vee}\right)$ is well-defined. For such $t$, let

$$
\mathcal{O}^{W_{\chi}^{s c}}\left(t \cdot Z\left(\bar{G}^{\vee}\right)\right)
$$

be the set of $W_{\chi}^{s c}$-orbits in $t \cdot Z\left(\bar{G}^{\vee}\right) \subset \bar{T}^{\vee}$.

Proposition 4.4. Assume $G$ is a semisimple group. Let $\chi$ be a unitary unramified genuine character of $Z(\bar{T})$. Then

$$
\left[R_{\chi}^{s c}: R_{\chi}\right]=\frac{\left|Z\left(\bar{G}^{\vee}\right)\right|}{\left|\mathcal{O}^{W_{\chi}^{s c}}\left(\phi_{\chi}^{\psi}(\varpi) \cdot Z\left(\bar{G}^{\vee}\right)\right)\right|} .
$$

Proof. For a finite group $H$ acting on a finite set $X$, the orbit counting formula reads

$$
|X / H|=\frac{1}{|H|} \sum_{h \in H}\left|X^{h}\right|
$$

where $X^{h} \subset X$ is the set of $h$-fixed points. To apply this to the case $X=\phi_{\chi}^{\psi}(\varpi) \cdot Z\left(\bar{G}^{\vee}\right)$ and $H=W_{\chi}^{s c}$, we first note that the action of $W_{\chi}^{s c}$ on the set $\phi_{\chi}^{\psi}(\varpi) \cdot Z\left(\bar{G}^{\vee}\right)$ is well-defined, as mentioned above. Since $W$ (and thus in particular $\left.W_{\chi}^{s c}\right)$ acts trivially on $Z\left(\bar{G}^{\vee}\right)$, we see

$$
X^{\mathrm{w}}= \begin{cases}X, & \text { if } \mathbb{w} \in W_{\chi} \\ \emptyset & \text { if } \mathbb{w} \notin W_{\chi}\end{cases}
$$

Thus,

$$
\left|\mathcal{O}^{W_{\chi}^{s c}}\left(\phi_{\chi}^{\psi}(\varpi) \cdot Z\left(\bar{G}^{\vee}\right)\right)\right|=\frac{1}{\left|W_{\chi}^{s c}\right|} \cdot\left|Z\left(\bar{G}^{\vee}\right)\right| \cdot\left|W_{\chi}\right| \cdot
$$

The result follows form the equality $\left[R_{\chi}^{s c}: R_{\chi}\right]=\left[W_{\chi}^{s c}: W_{\chi}\right]$. 
It is clear that the index $\left[R_{\chi}^{s c}: R_{\chi}\right]=\left[W_{\chi}^{s c}: W_{\chi}\right]$ is bounded above by $\left|Z\left(\bar{G}^{\vee}\right)\right|$ for covers of semisimple groups. In particular, if the dual group of $\bar{G}$ is of adjoint type, then $R_{\chi}^{s c}=R_{\chi}$. For example, if $G$ is a simply-connected Chevalley group and $n=1$, then there is no difference between $R_{\chi}^{s c}$ and $R_{\chi}$. For another nontrivial example, consider the $n$-fold cover $\overline{\mathrm{SL}}_{n+1}^{(n)}$, which has dual group $\mathrm{PGL}_{n+1}$, or the odd-degree cover of $\mathrm{Sp}_{2 r}$ whose dual group is $\mathrm{SO}_{2 r+1}$.

4.4. The group $R_{\chi}^{s c}$. Let $\bar{G}$ be an $n$-fold cover of a general linear algebraic group. Let $\mathbf{H}$ be the connected linear reductive group over $F$ such that its root datum is obtained from inverting that of $\overline{\mathbf{G}}_{Q, n}^{\vee}$, i.e., the Langlands dual group of $\mathbf{H}$ is isomorphic to $\overline{\mathbf{G}}_{Q, n}^{\vee}$. If $n=1$, then $\mathbf{H}=\mathbf{G}$. Let

$$
\mathbf{H}^{s c} \rightarrow \mathbf{H}_{\text {der }} \hookrightarrow \mathbf{H}
$$

be the simply-connected cover of the derived subgroup $\mathbf{H}_{d e r} \subset \mathbf{H}$. Thus, $Y_{Q, n}^{s c}$ is the cocharacter (and also the coroot) lattice of $\mathbf{H}^{s c}$. Denote by $H, H^{s c}$ the $F$-rational points of $\mathbf{H}$ and $\mathbf{H}^{s c}$ respectively. Here $H$ is the principal endoscopic group for $\bar{G}$.

We see that $T_{Q, n}^{s c}$ is just the torus of $H^{s c}$. The genuine character $\chi^{s c}$ of $Z(\bar{T})^{s c}$ gives rise to a linear unramified character

$$
\underline{\chi}^{s c}: T_{Q, n}^{s c} \rightarrow \mathbf{C}^{\times} \text {given by } \underline{\chi}^{s c}\left(\alpha_{Q, n}^{\vee}(a)\right):=\chi^{s c}\left(\bar{h}_{\alpha}\left(a^{n_{\alpha}}\right)\right)
$$

for all $\alpha \in \Delta$. In fact, the covering

$$
\mu_{n} \longleftrightarrow Z(\bar{T})^{s c} \longrightarrow T_{Q, n}^{s c, \dagger}
$$

has a splitting $\rho^{s c}$ given by $\alpha_{Q, n}^{\vee}(a) \mapsto \bar{h}_{\alpha}\left(a^{n_{\alpha}}\right)$ for all $\alpha \in \Delta$, and we have

$$
\underline{\chi}^{s c}=\chi^{s c} \circ \rho^{s c} \circ i^{s c} .
$$

One could form the unramified principal series $I\left(\underline{\chi}^{s c}\right)$ of $H^{s c}$ and thus have the $R$-group $R_{\underline{\chi}^{s c}}$ for $I\left(\underline{\chi}^{s c}\right)$.

Proposition 4.5. With notations as above, one has

$$
R_{\chi}^{s c} \simeq R_{\underline{\chi}^{s c}}
$$

Proof. It suffices to show that $W_{\chi^{s c}}=W_{\underline{\chi}^{s c}}$ and $\Phi_{\chi^{s c}}=\Phi_{\underline{\chi}^{s c}}$. By definition of $\underline{\chi}^{s c}$, we have

$$
\underline{\chi}^{s c}\left(\alpha_{Q, n}^{\vee}(a)\right)=\chi^{s c}\left(\bar{h}_{\alpha}\left(a^{n_{\alpha}}\right)\right)
$$

for all $\alpha \in \Delta$. We claim that the equality holds for all $\alpha \in \Phi$. By induction on the length of $\mathbb{w}$ such that $\mathbb{w}(\alpha) \in \Delta$, it suffices to prove that if

$$
\underline{\chi}^{s c}\left(\beta_{Q, n}^{\vee}(a)\right)=\chi^{s c}\left(\bar{h}_{\beta}\left(a^{n_{\beta}}\right)\right), \beta \in \Phi
$$

and $\gamma^{\vee}=\mathbb{w}_{\alpha}\left(\beta^{\vee}\right), \alpha \in \Delta$, then

$$
\underline{\chi}^{s c}\left(\gamma_{Q, n}^{\vee}(a)\right)=\chi^{s c}\left(\bar{h}_{\gamma}\left(a^{n_{\gamma}}\right)\right), \beta \in \Phi
$$

As shown in [Gao18, Page 112], one has

$$
\bar{h}_{\gamma}\left(a^{n_{\gamma}}\right)=w_{\alpha}^{-1} \cdot \bar{h}_{\beta}\left(a^{n_{\beta}}\right) \cdot w_{\alpha}=w_{\alpha} \cdot \bar{h}_{\beta}\left(a^{n_{\beta}}\right) \cdot w_{\alpha}^{-1},
$$


which is also equal to $\bar{h}_{\beta}\left(a^{n_{\beta}}\right) \cdot \bar{h}_{\alpha}\left(a^{n_{\alpha}}\right)^{-\left\langle\alpha_{Q, n}, \beta_{Q, n}^{\vee}\right\rangle}$ by (5). Thus by induction hypothesis, we have

$$
\begin{aligned}
\underline{\chi}^{s c}\left(\gamma_{Q, n}^{\vee}(a)\right) & =\underline{\chi}^{s c}\left(\beta_{Q, n}^{\vee}(a)\right) \cdot \underline{\chi}^{s c}\left(\alpha_{Q, n}^{\vee}(a)\right)^{-\left\langle\alpha_{Q, n}, \beta_{Q, n}^{\vee}\right\rangle} \\
& =\chi^{s c}\left(\bar{h}_{\beta}\left(a^{n_{\beta}}\right)\right) \cdot \chi^{s c}\left(\bar{h}_{\alpha}\left(a^{n_{\alpha}}\right)\right)^{-\left\langle\alpha_{Q, n}, \beta_{Q, n}^{\vee}\right\rangle} \\
& =\chi^{s c}\left(\bar{h}_{\gamma}\left(a^{n_{\gamma}}\right)\right) .
\end{aligned}
$$

This proves that the equality in (20) holds for all $\alpha \in \Phi$, and thus $\Phi_{\underline{\chi}^{s c}}=\Phi_{\chi^{s c}}$.

Let $\mathbb{w}=\mathbb{w}_{l} \mathbb{w}_{l-1} \ldots \mathbb{w}_{i}$ be a minimal decomposition of $\mathbb{w}$ with $\mathbb{w}_{i}=\mathbb{w}_{\alpha_{i}}$ for some $\alpha_{i} \in \Delta$. Let $w=w_{1} \ldots w_{2} w_{1}$, where $w_{i}:=w_{\alpha_{i}}$ be the representative of $\mathbb{w}_{\alpha_{i}}$. The above argument also shows inductively that

$$
w \cdot \bar{h}_{\alpha}\left(\varpi^{n_{\alpha}}\right) \cdot w^{-1}=\bar{h}_{\mathrm{w}(\alpha)}\left(\varpi^{n_{\mathrm{w}(\alpha)}}\right)
$$

for all $\alpha \in \Phi$. It follows that

$$
w^{-1} \cdot \bar{h}_{\alpha}\left(\varpi^{n_{\alpha}}\right) \cdot w=\bar{h}_{\mathrm{w}^{-1}(\alpha)}\left(\varpi^{n_{\mathrm{w}}-1(\alpha)}\right)
$$

for all $\alpha \in \Phi$, and thus

$$
{ }^{w}\left(\underline{\chi}^{s c}\right)\left(\alpha_{Q, n}^{\vee}(a)\right)={ }^{w}\left(\chi^{s c}\right)\left(\bar{h}_{\alpha}\left(a^{n_{\alpha}}\right)\right)
$$

for all $\mathbb{w} \in W$ and $\alpha \in \Delta$. Therefore, $W_{\underline{\chi}^{s c}}=W_{\chi^{s c}}$. Thus by the definition of $R$-groups, we have $R_{\underline{\chi}^{s c}} \simeq R_{\chi}^{s c}$.

In [Key82b, §3], all possibilities for the group $R_{\chi}^{s c} \simeq R_{\underline{\chi}^{s c}}$ are tabulated as in Table 1 and Table 2.

TABLE 1. The group $R_{\chi}^{s c}$ for classical group $\mathbf{H}^{s c}$

\begin{tabular}{|c|c|c|c|c|c|}
\hline $\mathbf{H}^{s c}$ & $A_{r}$ & $B_{r}$ & $C_{r}$ & $D_{r}, r$ even & $D_{r}, r$ odd \\
\hline$R_{\chi}^{s c}$ & $\mathbf{Z} / d \mathbf{Z}, d \mid(r+1)$ & $\mathbf{Z} / 2 \mathbf{Z}$ & $\mathbf{Z} / 2 \mathbf{Z}$ & $\mathbf{Z} / 2 \mathbf{Z}$ or $(\mathbf{Z} / 2 \mathbf{Z})^{2}$ & $\mathbf{Z} / 2 \mathbf{Z}$ or $\mathbf{Z} / 4 \mathbf{Z}$ \\
\hline
\end{tabular}

TABle 2. The group $R_{\chi}^{s c}$ for exceptional group $\mathbf{H}^{s c}$

\begin{tabular}{|c|c|c|c|c|c|}
\hline $\mathbf{H}^{s c}$ & $E_{6}$ & $E_{7}$ & $E_{8}$ & $F_{4}$ & $G_{2}$ \\
\hline$R_{\chi}^{s c}$ & $\mathbf{Z} / 3 \mathbf{Z}$ & $\mathbf{Z} / 2 \mathbf{Z}$ & $\{1\}$ & $\{1\}$ & $\{1\}$ \\
\hline
\end{tabular}

Immediately, one has

Theorem 4.6. Let $\bar{G}$ be an $n$-fold cover of a connected reductive group $G$, and $\chi$ a unitary unramified genuine character of $Z(\bar{T})$. Then $R_{\chi} \subset R_{\chi}^{s c}$ is abelian and therefore

$$
I(\chi)=\bigoplus_{\sigma \in \operatorname{Irr}\left(R_{\chi}\right)} \pi_{\sigma}
$$

i.e., the decomposition of $I(\chi)$ is multiplicity-free.

Corollary 4.7. For every $\mathbb{w} \in R_{\chi}$, one has

$$
\mathscr{A}(w, \chi)=\bigoplus_{\sigma \in \operatorname{Irr}\left(R_{\chi}\right)} \sigma(\mathbb{w}) \cdot \mathrm{id}_{\pi_{\sigma}} .
$$

Therefore, for every $f \in C_{c}^{\infty}(\bar{G})$,

$$
\text { Trace } \mathscr{A}(w, \chi) I(\chi)(f)=\sum_{\sigma \in \operatorname{Irr}\left(R_{\chi}\right)} \sigma(\mathbb{w}) \cdot \text { Trace } \pi_{\sigma}(f) \text {. }
$$


Proof. It suffices to verify the first equality, as the second will follow from it. For $\sigma \in$ $\operatorname{Irr}\left(R_{\chi}\right)$, recall the projection $P_{\sigma}$ of $I(\chi)$ on $\pi_{\sigma}$. By Schur's Lemma, $\mathscr{A}(w, \chi)$ acts on $\pi_{\sigma}$ as a homothety given by $c_{\pi_{\sigma}}(\mathbb{w}) \in \mathbf{C}$. However,

$$
\begin{aligned}
P_{\sigma^{\prime}} & =\frac{1}{\left|R_{\chi}\right|} \sum_{\mathbb{w} \in R_{\chi}} \overline{\sigma^{\prime}(\mathbb{w})}\left(\bigoplus_{\sigma \in \operatorname{Irr}\left(R_{\chi}\right)} c_{\pi_{\sigma}}(\mathbb{w}) \cdot \mathrm{id}_{\pi_{\sigma}}\right) \\
& =\bigoplus_{\sigma \in \operatorname{Irr}\left(R_{\chi}\right)}\left(\frac{1}{\left|R_{\chi}\right|} \sum_{\mathbb{w} \in R_{\chi}} \overline{\sigma^{\prime}(\mathbb{w})} \cdot c_{\pi_{\sigma}}(\mathbb{w})\right) \cdot \mathrm{id}_{\pi_{\sigma}} .
\end{aligned}
$$

That is, the pairing of the class function $c_{\pi_{\sigma}}(-)$ on $R_{\chi}$ with $\sigma^{\prime}(-)$ is equal to the Kronecker delta function $\delta_{\sigma, \sigma^{\prime}}$. Since the characters $\operatorname{Irr}\left(R_{\chi}\right)$ form an orthonormal basis for class functions on $R_{\chi}$, this shows that $c_{\pi_{\sigma}}=\sigma$ for every $\sigma \in \operatorname{Irr}\left(R_{\chi}\right)$.

4.5. Covers of $\mathrm{SL}_{2}$. We illustrate the previous discussion on R-group by considering the $n$-fold cover $\bar{G}=\overline{\mathrm{SL}}_{2}^{(n)}$ arising from $Q\left(\alpha^{\vee}\right)=1$. We show that our analysis agrees with certain results in [Szp19].

If $n$ is even, then the dual group $\bar{G}^{\vee}$ is $\mathrm{SL}_{2}$. Write

$$
s_{\zeta}:=\phi_{\chi}^{\psi}(\varpi)=\left(\begin{array}{cc}
\zeta & \\
& \zeta^{-1}
\end{array}\right) \in \bar{G}^{\vee}
$$

for the relative Satake parameter of $\chi$ discussed in $\S 4.3$, where $\zeta \in \mathbf{C}^{\times}$depends on $\chi$. There are three cases:

- $\zeta^{4} \neq 1$. In this case, $\Phi_{\chi}=\emptyset$ and thus $W\left(\Phi_{\chi}\right)=W$. Also $W_{\chi}^{s c}=W_{\chi}=\{1\}$. Therefore $R_{\chi}^{s c}=R_{\chi}=\{1\}$, and in particular $\left|\mathcal{O}^{W_{\chi}^{s c}}\left(s_{\zeta} \cdot Z\left(\bar{G}^{\vee}\right)\right)\right|=2$.

- $\zeta^{4}=1$ but $\zeta^{2} \neq 1$. In this case, $\Phi_{\chi}=\emptyset$ and thus $W\left(\Phi_{\chi}\right)=W$. However, we have $W_{\chi}^{s c}=W$, while $W_{\chi}=\{1\}$. Therefore $R_{\chi}^{s c}=W$ and $R_{\chi}=\{1\}$. Indeed, we have $\left|\mathcal{O}^{W_{\chi}^{s c}}\left(s_{\zeta} \cdot Z\left(\bar{G}^{\vee}\right)\right)\right|=1$ in this case.

- $\zeta^{2}=1$. In this case, $\Phi_{\chi}=\{\alpha\}$ and thus $W\left(\Phi_{\chi}\right)=\{1\}$. Also $W_{\chi}^{s c}=W_{\chi}=W$. Therefore $R_{\chi}^{s c}=R_{\chi}=\{1\}$, and also $\left|\mathcal{O}^{W_{\chi}^{s c}}\left(s_{\zeta} \cdot Z\left(\bar{G}^{\vee}\right)\right)\right|=2$.

Hence for $n$ even, $I(\chi)$ is always irreducible for a unitary unramified genuine character $\chi$.

Now we consider $n$ odd, in which case the dual group of $\overline{\mathrm{SL}}_{2}^{(n)}$ is $\bar{G}^{\vee}=\mathrm{PGL}_{2}$ and thus one always has $R_{\chi}^{s c}=R_{\chi}$ by Proposition 4.4. Write

$$
s_{\zeta}:=\rho_{\chi}^{\psi}(\varpi)=\left(\begin{array}{cc}
\zeta & \\
& 1
\end{array}\right) \in \bar{G}^{\vee}
$$

for the relative Satake parameter for $I(\chi)$. There are three cases:

- $\zeta^{2} \neq 1$. In this case, $\Phi_{\chi}=\emptyset$ and thus $W\left(\Phi_{\chi}\right)=W$. Also $W_{\chi}=\{1\}$. Therefore $R_{\chi}=\{1\}$.

- $\zeta=-1$. Then $\Phi_{\chi}=\emptyset$ and thus $W\left(\Phi_{\chi}\right)=W$. But $W_{\chi}=W$. Therefore $R_{\chi}=W$.

- $\zeta=1$. Then $\Phi_{\chi}=\{\alpha\}$ and thus $W\left(\Phi_{\chi}\right)=\{1\}$. But $W_{\chi}=W$. In this case, $R_{\chi}=\{1\}$.

Combining the above even and odd cases, we see that the only reducibility point for $I(\chi)$ is when $n$ is odd and $\chi$ is such that $\underline{\chi}_{\alpha}$ is a nontrivial quadratic character; in this case

$$
I(\chi)=\pi_{\chi}^{u n} \oplus \pi
$$

The result agrees with [Szp19, Proposition 5.1]. 
4.6. Comparison of $R_{\chi}$ and $\mathcal{S}_{\phi_{\chi}}$. For linear algebraic groups, it was shown by Keys [Key87] that the R-group $R_{\chi}$ is naturally identified with the component group of the centralizer of the parameter $\phi_{\chi}$. In this subsection, we establish the same identification for covering groups.

Let

$$
\phi: W_{F} \rightarrow{ }^{L} \bar{G}
$$

be an $L$-parameter. Let $S_{\phi} \subset \bar{G}^{\vee}$ be the centralizer of $\phi\left(W_{F}\right)$ in $\bar{G}^{\vee}$, i.e.,

$$
S_{\phi}:=\left\{g \in \bar{G}^{\vee}: g \cdot \phi(a) \cdot g^{-1}=\phi(a) \text { for every } a \in W_{F}\right\}
$$

it is a reductive subgroup of $\bar{G}^{\vee}$ but not necessarily connected. Let $S_{\phi}^{0}$ be the connected component of $S_{\phi}$. Define

$$
\mathcal{S}_{\phi}:=\frac{S_{\phi}}{Z\left(\bar{G}^{\vee}\right) \cdot S_{\phi}^{0}} .
$$

There is a dual group (instead of $L$-group) relative version which is more closely related to linear algebraic groups. Recall that depending on the choice of a distinguished genuine character $\chi_{\psi}$, one has an isomorphism

$$
{ }^{L} \bar{G} \simeq{ }_{\chi_{\psi}} \bar{G}^{\vee} \times W_{F},
$$

see (8). One obtains an unramified parameter

$$
\phi\left[\chi_{\psi}\right]: W_{F} \stackrel{\phi}{\longrightarrow}{ }^{L} \bar{G} \longrightarrow \bar{G}^{\vee},
$$

where the second map is the projection depending on $\chi_{\psi}$. Local Langlands correspondence gives the $L$-parameter $\phi_{\chi_{\psi}}$ associated to $\chi_{\psi}$, which takes values in $Z\left(\bar{G}^{\vee}\right)$. One has

$$
\phi\left[\chi_{\psi}\right]=\phi \cdot \phi_{\chi_{\psi}}^{-1} .
$$

Analogously, let $S_{\phi\left[\chi_{\psi}\right]} \subset \bar{G}^{\vee}$ be the centralizer of $\phi\left[\chi_{\psi}\right]\left(W_{F}\right)$ in $\bar{G}^{\vee}$. Define

$$
\mathcal{S}_{\phi\left[\chi_{\psi}\right]}:=\frac{S_{\phi\left[\chi_{\psi}\right]}}{Z\left(\bar{G}^{\vee}\right) \cdot S_{\phi\left[\chi_{\psi}\right]}^{0}} .
$$

Lemma 4.8. With notations as above, $S_{\phi}=S_{\phi\left[\chi_{\psi}\right]}$ for every distinguished genuine character $\chi_{\psi}$. Hence, $\mathcal{S}_{\phi}=\mathcal{S}_{\phi\left[\chi_{\psi}\right]}$ as well.

Proof. It follows from the isomorphism ${ }^{L} \bar{G} \simeq_{\chi_{\psi}} \bar{G}^{\vee} \times W_{F}$ that

$$
\phi(a)=\left(\phi\left[\chi_{\psi}\right](a), a\right) \in \bar{G}^{\vee} \times W_{F} .
$$

Thus, $g \in \bar{G}^{\vee}$ centralizes $\phi(a)$ if and only if it centralizes $\phi\left[\chi_{\psi}\right](a)$; this gives the equality $S_{\phi}=S_{\phi\left[\chi_{\psi}\right]}$ and completes the proof.

Theorem 4.9. Let $\bar{G}$ be an $n$-fold cover of a connected reductive group $G$. Let $\chi$ be a unitary unramified genuine character of $Z(\bar{T})$, and let $\phi_{\chi}$ be the L-parameter associated to $\chi$. One has an isomorphism

$$
R_{\chi} \simeq \mathcal{S}_{\phi_{\chi}}
$$

Proof. The idea is to reduce it to the linear algebraic case where the isomorphism is proved in [Key87, Page 42].

As in $\S 4.4$, let $\mathbf{H}$ be the connected split linear algebraic group whose dual group is $\overline{\mathbf{G}}_{Q, n}^{\vee}$. Let $\mathbf{T}_{Q, n}$ be its split torus whose cocharacter lattice is $Y_{Q, n}$. We have $H, T_{Q, n}$ denoting the $F$-rational points of $\mathbf{H}, \mathbf{T}_{Q, n}$ respectively. 
Let $\chi$ be a unitary unramified genuine character of $Z(\bar{T})$. For simplicity, denote by $\phi$ (instead of $\phi_{\chi}$ ) the associated $L$-parameter valued in ${ }^{L} \bar{T}$. By choosing a distinguished genuine character $\chi_{\psi}$, we have the unramified parameter

$$
\phi\left[\chi_{\psi}\right]: W_{F} \rightarrow \bar{T}^{\vee} \hookrightarrow \bar{G}^{\vee},
$$

which is just $\phi_{\chi}^{\psi}$ in the notation of $\S 4.3$. Note that $\bar{T}^{\vee}=T_{Q, n}^{\vee}$ and $\bar{G}^{\vee}=H^{\vee}$. The parameter $\phi\left[\chi_{\psi}\right]$ is thus associated to an unramified character

$$
\underline{\chi}: T_{Q, n} \rightarrow \mathbf{C}^{\times}
$$

such that

$$
\phi_{\underline{\chi}}=\phi\left[\chi_{\psi}\right]
$$

where $\phi_{\underline{\chi}}$ is the $L$-parameter associated to $\underline{\chi}$ by the local Langlands correspondence for linear torus. We can explicate $\underline{\chi}$ as follows. First, $\chi \cdot \chi_{\psi}^{-1}: T_{Q, n}^{\dagger} \rightarrow \mathbf{C}^{\times}$is a linear character, where $T_{Q, n}^{\dagger}$ is the image of the isogeny

$$
i_{Q, n}: T_{Q, n} \rightarrow T
$$

see $\S 4.2$. Then, $\underline{\chi}$ is just the pull-back of $\chi \cdot \chi_{\psi}^{-1}$ via $i_{Q, n}$; that is, $\underline{\chi}=\left(\chi \cdot \chi_{\psi}^{-1}\right) \circ i_{Q, n}$. In any case, we have

$$
\mathcal{S}_{\phi_{\underline{\chi}}}=\mathcal{S}_{\phi\left[\chi_{\psi}\right]} .
$$

Now we consider $R_{\underline{\chi}}$ and $R_{\chi}$. Since $\chi_{\psi}$ is Weyl-invariant, we have

$$
W_{\chi}=W_{\chi \cdot \chi_{\psi}^{-1}}=W_{\underline{\chi}} .
$$

By the construction of distinguished genuine character, we have

$$
\chi_{\psi}\left(\bar{h}_{\alpha}\left(a^{n_{\alpha}}\right)\right)=1
$$

for all $\alpha \in \Delta$ (see [GG18, §6.1]). However, from the proof of Proposition 4.5 one has $w \cdot \bar{h}_{\alpha}\left(\varpi^{n_{\alpha}}\right) \cdot w^{-1}=\bar{h}_{\mathrm{w}(\alpha)}\left(\varpi^{n_{\mathrm{w}(\alpha)}}\right)$ for all $\mathbb{w} \in W$ and $\alpha \in \Delta$. It follows that $\chi_{\psi}\left(\bar{h}_{\alpha}\left(a^{n_{\alpha}}\right)\right)=$ 1 for every $\alpha \in \Phi$. Thus,

$$
\begin{aligned}
\Phi_{\underline{\chi}} & =\left\{\alpha_{Q, n}>0: \underline{\chi}\left(a^{\alpha_{Q, n}^{\vee}}\right)=1\right\} \\
& =\left\{\alpha>0:\left(\chi \cdot \chi_{\psi}^{-1}\right)\left(\bar{h}_{\alpha}\left(a^{n_{\alpha}}\right)\right)=1\right\} \\
& =\left\{\alpha>0: \chi\left(\bar{h}_{\alpha}\left(a^{n_{\alpha}}\right)\right)=1\right\} \\
& =\left\{\alpha>0: \underline{\chi}_{\alpha}=\mathbb{1}\right\} \\
& =\Phi_{\chi} .
\end{aligned}
$$

We deduce from (22) and (23) that

$$
R_{\underline{\chi}} \simeq R_{\chi}
$$

It is proved in [Key87, Proposition 2.6] that one has the isomorphism $R_{\underline{\chi}} \simeq \mathcal{S}_{\phi_{\underline{\chi}}}$. From this we see that $R_{\chi} \simeq \mathcal{S}_{\phi\left[\chi_{\psi}\right]}$, which is also isomorphic to $\mathcal{S}_{\phi}$ by Lemma 4.8. This completes the proof.

In view of the isomorphism $R_{\chi} \simeq \mathcal{S}_{\phi_{\chi}}$, the character relation in Corollary 4.7 can be interpreted in terms of $\operatorname{Irr}\left(\mathcal{S}_{\phi_{\chi}}\right)$ as well.

Corollary 4.10. If the dual group $\bar{G}^{\vee}$ is semisimple simply-connected, then $R_{\chi}=\{1\}$ for every unitary unramified genuine character $\chi$ of $Z(\bar{T})$. 
Proof. This follows from the isomorphism $R_{\chi} \simeq \mathcal{S}_{\phi_{\chi}}$ and the well-known result of Steinberg that the centralizer of a semisimple element inside a simply-connected group (such as $\bar{G}^{\vee}$ by our assumption) is connected.

Alternatively (and equivalently), from the proof of the preceding theorem, we have $R_{\chi}=R_{\underline{\chi}}$, where $I(\underline{\chi})$ is an unramified principal series of $H$. If $\bar{G}^{\vee}$ is simply-connected, then $H$ is of adjoint type and it can be argued directly (see [Li92, Corollary 1.6] or [BM94]) that $R_{\underline{\chi}}=\{1\}$ in this case.

Example 4.11. Let $\mathbf{G}=\mathrm{Sp}_{2 r}$ and let $Q$ be a Weyl-invariant quadratic form on its cocharacter lattice. If $n_{\alpha}=0(\bmod 2)$ for the unique short simple coroot $\alpha^{\vee}$ of $\mathrm{Sp}_{2 r}$, then

$$
\bar{G}^{\vee}=\mathrm{Sp}_{2 r}
$$

in this case $R_{\chi}=\{1\}$ and $I(\chi)$ is always irreducible. This applies in particular to the case of metaplectic-type group $\overline{\mathrm{Sp}}_{2 r}^{(n)}$, i.e., when $n_{\alpha}=2 \bmod 4$, see Definition 2.1. For $\overline{\mathrm{SL}}_{2}^{(n)}$, this is compatible with the discussion in $\S 4.5$.

\section{WhitTAKER SPACE AND THE MAIN CONJECTURE}

The main goal of this section is to investigate $\operatorname{dim} \mathrm{Wh}_{\psi}(\pi)$, where $\pi \in \Pi(\chi)$ is any irreducible constituent of $I(\chi)$.

5.1. The Whittaker space. Let $\psi: F \rightarrow \mathbf{C}^{\times}$be an additive character of conductor $O$. Let

$$
\psi_{U}: U \rightarrow \mathbf{C}^{\times}
$$

be the character of $U$ such that its restriction to every $U_{\alpha}, \alpha \in \Delta$ is given by $\psi \circ e_{\alpha}^{-1}$. We may write $\psi$ instead of $\psi_{U}$ for simplicity.

Definition 5.1. For a genuine representation $\left(\pi, V_{\pi}\right)$ of $\bar{G}$, a linear functional $\ell: V_{\pi} \rightarrow \mathbf{C}$ is called a $\psi$-Whittaker functional if $\ell(\pi(u) v)=\psi(u) \cdot v$ for all $u \in U$ and $v \in V_{\pi}$. Write $\mathrm{Wh}_{\psi}(\pi)$ for the space of $\psi$-Whittaker functionals for $\pi$.

The space $\mathrm{Wh}_{\psi}(I(\chi))$ for an unramified principal series $I(\chi)$ could be described as follows.

- First, let $\mathrm{Ftn}(i(\chi))$ be the vector space of functions $\mathbf{c}$ on $\bar{T}$ satisfying

$$
\mathbf{c}(\bar{t} \cdot \bar{z})=\mathbf{c}(\bar{t}) \cdot \chi(\bar{z}) \text { for } \bar{t} \in \bar{T} \text { and } \bar{z} \in \bar{A} \text {. }
$$

The support of any $\mathbf{c} \in \mathrm{Ftn}(i(\chi))$ is a disjoint union of cosets in $\bar{T} / \bar{A}$. We have

$$
\operatorname{dim} \operatorname{Ftn}(i(\chi))=\left|\mathscr{X}_{Q, n}\right| \text {, }
$$

since $\bar{T} / \bar{A} \simeq Y / Y_{Q, n}=\mathscr{X}_{Q, n}$.

- Second, for every $\gamma \in \bar{T}$, let $\mathbf{c}_{\gamma} \in \operatorname{Ftn}(i(\chi))$ be the unique element satisfying

$$
\operatorname{supp}\left(\mathbf{c}_{\gamma}\right)=\gamma \cdot \bar{A} \text { and } \mathbf{c}_{\gamma}(\gamma)=1 \text {. }
$$

Clearly, $\mathbf{c}_{\gamma \cdot a}=\chi(a)^{-1} \cdot \mathbf{c}_{\gamma}$ for every $a \in \bar{A}$. If $\left\{\gamma_{i}\right\} \subset \bar{T}$ is a chosen set of representatives of $\bar{T} / \bar{A}$, then $\left\{\mathbf{c}_{\gamma_{i}}\right\}$ forms a basis for $\operatorname{Ftn}(i(\chi))$. Let $i(\chi)^{\vee}$ be the vector space of functionals of $i(\chi)$. The set $\left\{\gamma_{i}\right\}$ gives rise to linear functionals $l_{\gamma_{i}} \in i(\chi)^{\vee}$ such that $l_{\gamma_{i}}\left(\phi_{\gamma_{j}}\right)=\delta_{i j}$, where $\phi_{\gamma_{j}} \in i(\chi)$ is the unique element such that

$$
\operatorname{supp}\left(\phi_{\gamma_{j}}\right)=\bar{A} \cdot \gamma_{j}^{-1} \text { and } \phi_{\gamma_{j}}\left(\gamma_{j}^{-1}\right)=1 .
$$

It is easy to see that for any $\gamma \in \bar{T}$ and $a \in \bar{A}$, one has

$$
\phi_{\gamma a}=\chi(a) \cdot \phi_{\gamma}, \quad l_{\gamma a}=\chi(a)^{-1} \cdot l_{\gamma} .
$$


Moreover, there is a natural isomorphism of vector spaces

$$
\operatorname{Ftn}(i(\chi)) \simeq i(\chi)^{\vee}
$$

given by

$$
\mathbf{c} \mapsto l_{\mathbf{c}}:=\sum_{\gamma_{i} \in \bar{T} / \bar{A}} \mathbf{c}\left(\gamma_{i}\right) \cdot l_{\gamma_{i}} .
$$

It can be checked easily that this isomorphism does not depend on the choice of representatives for $\bar{T} / \bar{A}$.

- Third, there is an isomorphism between $i(\chi)^{\vee}$ and the $\operatorname{space}^{\mathrm{Wh}_{\psi}}(I(\chi))$ of $\psi$ Whittaker functionals of $I(\chi)$ (see [McN16, $\S 6]$ ), given by $\lambda \mapsto W_{\lambda}$ with

$$
W_{\lambda}: I(\chi) \rightarrow \mathbf{C}, \quad f \mapsto \lambda\left(\int_{U} f\left(\bar{w}_{G}^{-1} u\right) \psi(u)^{-1} \mu(u)\right)
$$

where $f \in I(\chi)$ is an $i(\chi)$-valued function on $\bar{G}$. Here $\bar{w}_{G}=\bar{w}_{\alpha_{1}} \bar{w}_{\alpha_{2}} \ldots \bar{w}_{\alpha_{k}} \in K$ is the representative of $\mathbb{w}_{G}$, where $\mathbb{w}_{G}=\mathbb{w}_{\alpha_{1}} \mathbb{w}_{\alpha_{2}} \ldots \mathbb{w}_{\alpha_{k}}$ is a minimum decomposition of $\mathbb{w}_{G}$.

Thus, we have a chain of natural isomorphisms of vector spaces all of dimension $\left|\mathscr{X}_{Q, n}\right|$ :

$$
\operatorname{Ftn}(i(\chi)) \simeq i(\chi)^{\vee} \simeq \mathrm{Wh}_{\psi}(I(\chi)) .
$$

For every $\mathbf{c} \in \mathrm{Ftn}(i(\chi))$, by abuse of notation, we will write $\lambda_{\mathbf{c}}^{\chi} \in \mathrm{Wh}_{\psi}(I(\chi))$ for the resulting $\psi$-Whittaker functional of $I(\chi)$ obtained from the above isomorphism.

The operator $A(w, \chi): I(\chi) \rightarrow I\left({ }^{w} \chi\right)$ induces a homomorphism of vector spaces

$$
A(w, \chi)^{*}: \mathrm{Wh}_{\psi}\left(I\left({ }^{w} \chi\right)\right) \rightarrow \mathrm{Wh}_{\psi}(I(\chi))
$$

given by

$$
\left\langle\lambda_{\mathbf{c}}^{w} \chi,-\right\rangle \mapsto\left\langle\lambda_{\mathbf{c}}^{w} \chi, A(w, \chi)(-)\right\rangle
$$

where $\mathbf{c} \in \operatorname{Ftn}\left(i\left({ }^{w} \chi\right)\right)$.

5.2. The scattering matrix $\mathcal{S}_{\mathfrak{R}}(w, i(\chi))$. Let $\mathfrak{R}, \mathfrak{R}^{\prime} \subset \bar{T}$ be two ordered sets of representatives of $\bar{T} / \bar{A}=\mathscr{X}_{Q, n}$. Let

$$
\left\{\lambda_{\gamma}^{w} \chi: \gamma \in \mathfrak{R}\right\}
$$

be the ordered basis for $\mathrm{Wh}_{\psi}\left(I\left({ }^{w} \chi\right)\right)$, and

$$
\left\{\lambda_{\gamma^{\prime}}^{\chi}: \gamma^{\prime} \in \mathfrak{R}^{\prime}\right\}
$$

the ordered basis for $\mathrm{Wh}_{\psi}(I(\chi))$. The map $A(w, \chi)^{*}$ is then determined by the so-called scattering matrix

$$
\mathcal{S}_{\mathfrak{R}, \Re^{\prime}}(w, i(\chi))=\left[\tau\left(w, \chi, \gamma, \gamma^{\prime}\right)\right]_{\gamma \in \mathfrak{R}, \gamma^{\prime} \in \mathfrak{R}^{\prime}}
$$

such that

$$
A(w, \chi)^{*}\left(\lambda_{\gamma}^{w} \chi\right)=\sum_{\gamma^{\prime} \in \mathfrak{R}^{\prime}} \tau\left(w, \chi, \gamma, \gamma^{\prime}\right) \cdot \lambda_{\gamma^{\prime}}^{\chi}
$$

We briefly describe the matrix $\mathcal{S}_{\mathfrak{R}, \mathfrak{R}^{\prime}}(w, i(\chi))$. First, we have:

- For $\mathbb{w} \in W$ and $\bar{z}, \bar{z}^{\prime} \in \bar{A}$, the identity

$$
\tau\left(w, \chi, \gamma \cdot \bar{z}, \gamma^{\prime} \cdot \bar{z}^{\prime}\right)=\left({ }^{w} \chi\right)^{-1}(\bar{z}) \cdot \tau\left(w, \chi, \gamma, \gamma^{\prime}\right) \cdot \chi\left(\bar{z}^{\prime}\right)
$$

holds. 
- For $\mathbb{w}_{1}, \mathbb{w}_{2} \in W$ such that $l\left(\mathbb{w}_{2} \mathbb{w}_{1}\right)=l\left(\mathbb{w}_{2}\right)+l\left(\mathbb{w}_{1}\right)$, one has

$$
\tau\left(w_{2} w_{1}, \chi, \gamma, \gamma^{\prime}\right)=\sum_{\gamma^{\prime \prime} \in \bar{T} / \bar{A}} \tau\left(w_{2},{ }^{w_{1}} \chi, \gamma, \gamma^{\prime \prime}\right) \cdot \tau\left(w_{1}, \chi, \gamma^{\prime \prime}, \gamma^{\prime}\right)
$$

which is referred to as the cocycle relation. By (25), the above sum is independent of the choice of representatives $\gamma^{\prime \prime}$.

The above cocycle relation implies that in principle it suffices to understand $\tau\left(w_{\alpha}, \chi, \gamma, \gamma^{\prime}\right)$ for $\gamma, \gamma^{\prime} \in \bar{T}$ for $\alpha \in \Delta$. For this purpose, let $d u$ be the self-dual Haar measure of $F$ with respect to $\psi$ such that $d u(O)=1$; thus, $d u\left(O^{\times}\right)=1-q^{-1}$. Consider the Gauss sum

$$
G_{\psi}(a, b):=\int_{O^{\times}}(u, \varpi)_{n}^{a} \cdot \psi\left(\varpi^{b} u\right) d u \text { for } a, b \in \mathbf{Z} .
$$

We also write

$$
\mathbf{g}_{\psi}(k):=G_{\psi}(k,-1),
$$

where $k \in \mathbf{Z}$ is any integer. Denote henceforth

$$
\varepsilon:=(-1, \varpi)_{n} \in \mathbf{C}^{\times} .
$$

It is known that

$$
\mathbf{g}_{\psi}(k)= \begin{cases}\varepsilon^{k} \cdot \overline{\mathbf{g}_{\psi}(-k)} & \text { for every } k \in \mathbf{Z}, \\ -q^{-1} & \text { if } n \mid k, \\ \mathbf{g}_{\psi}(k) & \text { with }\left|\mathbf{g}_{\psi}(k)\right|=q^{-1 / 2} \text { if } n \nmid k .\end{cases}
$$

Here $\bar{z}$ denotes the complex conjugation of a complex number $z$.

It is shown in [KP84,McN16] (with some refinement from [Gao17]) that $\tau\left(w_{\alpha}, \chi, \gamma, \gamma^{\prime}\right)$ is determined as follows.

Theorem 5.2. Suppose that $\gamma=\mathbf{s}_{y_{1}}$ and $\gamma^{\prime}=\mathbf{s}_{y}$ with $y_{1}, y \in Y$. First, we can write $\tau\left(w_{\alpha}, \chi, \gamma, \gamma^{\prime}\right)=\tau^{1}\left(w_{\alpha}, \chi, \gamma, \gamma^{\prime}\right)+\tau^{2}\left(w_{\alpha}, \chi, \gamma, \gamma^{\prime}\right)$ satisfying the following properties:

- $\tau^{i}\left(w_{\alpha}, \chi, \gamma \cdot \bar{z}, \gamma^{\prime} \cdot \bar{z}^{\prime}\right)=\left({ }^{w_{\alpha}} \chi\right)^{-1}(\bar{z}) \cdot \tau^{i}\left(w_{\alpha}, \chi, \gamma, \gamma^{\prime}\right) \cdot \chi\left(\bar{z}^{\prime}\right)$ for all $\bar{z}, \bar{z}^{\prime} \in \bar{A}$;

- $\tau^{1}\left(w_{\alpha}, \chi, \gamma, \gamma^{\prime}\right)=0$ unless $y_{1} \equiv y \bmod Y_{Q, n}$;

- $\tau^{2}\left(w_{\alpha}, \chi, \gamma, \gamma^{\prime}\right)=0$ unless $y_{1} \equiv \mathbb{w}_{\alpha}[y] \bmod Y_{Q, n}$.

Second,

- if $y_{1}=y$, then

$$
\tau^{1}\left(w_{\alpha}, \chi, \gamma, \gamma^{\prime}\right)=\left(1-q^{-1}\right) \frac{\chi\left(\bar{h}_{\alpha}\left(\varpi^{n_{\alpha}}\right)\right)^{k_{y, \alpha}}}{1-\chi\left(\bar{h}_{\alpha}\left(\varpi^{n_{\alpha}}\right)\right)}, \text { where } k_{y, \alpha}=\left\lceil\frac{\langle y, \alpha\rangle}{n_{\alpha}}\right\rceil ;
$$

- if $y_{1}=\mathbb{w}_{\alpha}[y]$, then

$$
\tau^{2}\left(w_{\alpha}, \chi, \gamma, \gamma^{\prime}\right)=\varepsilon^{\left\langle y_{\rho}, \alpha\right\rangle \cdot D\left(y, \alpha^{\vee}\right)} \cdot \mathbf{g}_{\psi^{-1}}\left(\left\langle y_{\rho}, \alpha\right\rangle Q\left(\alpha^{\vee}\right)\right) .
$$

5.3. The main conjecture. If $\mathfrak{R}=\mathfrak{R}^{\prime}$, then

$$
\mathcal{S}_{\mathfrak{R}}(w, i(\chi)):=\mathcal{S}_{\mathfrak{R}, \mathfrak{R}}(w, i(\chi)),
$$

is a square matrix with entires indexed by a single ordered set $\mathfrak{R}$. Since $W$ acts on $\mathscr{X}_{Q, n}=Y / Y_{Q, n}$ with respect to $\mathbb{w}[-]$, one has a decomposition

$$
\mathscr{X}_{Q, n}=\bigsqcup_{\mathcal{O}_{y} \in \mathcal{O}_{\mathscr{X}}} \mathcal{O}_{y}
$$

into $W$-orbits. This gives a natural partition

$$
\mathfrak{R}=\bigsqcup_{\mathcal{O}_{y} \in \mathcal{O}_{\mathscr{X}}} \mathfrak{R}_{y},
$$


where $\mathfrak{R}_{y} \subset \mathfrak{R}$ is the subset of representatives of $\mathcal{O}_{y}$. For each $W$-orbit $\mathcal{O}_{y}$, denote

$$
\mathcal{S}_{\mathfrak{R}}(w, i(\chi))_{\mathcal{O}_{y}}:=\left[\tau\left(w, \chi, \mathbf{s}_{z}, \mathbf{s}_{z^{\prime}}\right)\right]_{z, z^{\prime} \in \Re_{y}} .
$$

It follows from the cocycle relation $(26)$ and Theorem 5.2 that $\mathcal{S}_{\mathfrak{R}}(w, i(\chi))$ is a blockdiagonal matrix with blocks $\mathcal{S}_{\mathfrak{R}}(w, i(\chi))_{\mathcal{O}_{y}}$ for $\mathcal{O}_{y} \in \mathcal{O}_{\mathscr{X}}$, which we write as

$$
\mathcal{S}_{\mathfrak{R}}(w, i(\chi))=\bigoplus_{\mathcal{O}_{y} \in \mathcal{O}_{\mathscr{C}}} \mathcal{S}_{\mathfrak{R}}(w, i(\chi))_{\mathcal{O}_{y}} .
$$

In fact, for $y \in \mathscr{X}_{Q, n}$, let

$$
\mathrm{Wh}_{\psi}(I(\chi))_{\mathcal{O}_{y}}=\operatorname{Span}\left\{\lambda_{\mathbf{s}_{z}}^{\chi}: z \in \mathfrak{R}_{y}\right\} \subset \mathrm{Wh}_{\psi}(I(\chi))
$$

be the "O $\mathcal{O}_{y}$-subspace" of the Whittaker space of $I(\chi)$. It is well-defined and independent of the representatives for $y \in \mathscr{X}_{Q, n}$, and moreover

$$
\operatorname{dim} \mathrm{Wh}_{\psi}(I(\chi))_{\mathcal{O}_{y}}=\left|\mathcal{O}_{y}\right| \text {. }
$$

One has a decomposition

$$
\mathrm{Wh}_{\psi}(I(\chi))=\bigoplus_{\mathcal{O}_{y} \in \mathcal{O}_{\mathscr{X}}} \mathrm{Wh}_{\psi}(I(\chi))_{\mathcal{O}_{y}}
$$

For every $\sigma \in \operatorname{Irr}\left(R_{\chi}\right)$, the inclusion $h_{\sigma}: \pi_{\sigma} \hookrightarrow I(\chi)$ induces a surjection of vector spaces

$$
h_{\sigma}^{*}: \mathrm{Wh}_{\psi}(I(\chi)) \rightarrow \mathrm{Wh}_{\psi}\left(\pi_{\sigma}\right) \text {. }
$$

Denote

$$
\mathrm{Wh}_{\psi}\left(\pi_{\sigma}\right)_{\mathcal{O}_{y}}:=h_{\sigma}^{*}\left(\mathrm{Wh}_{\psi}(I(\chi))_{\mathcal{O}_{y}}\right)
$$

Consider the natural permutation representation

$$
\sigma^{\mathscr{X}}: W \longrightarrow \operatorname{Perm}\left(\mathscr{X}_{Q, n}\right)
$$

of $W$ given by $\sigma_{\mathscr{X}}(\mathbb{w})(y)=\mathbb{w}[y]$. Clearly, one has a decomposition

$$
\sigma^{\mathscr{X}}=\bigoplus_{\mathcal{O}_{y} \in \mathcal{O}_{\mathscr{X}}} \sigma_{\mathcal{O}_{y}}^{\mathscr{X}},
$$

where

$$
\sigma_{\mathcal{O}_{y}}^{\mathscr{X}}: W \longrightarrow \operatorname{Perm}\left(\mathcal{O}_{y}\right)
$$

is the permutation representation on the $W$-orbit $\mathcal{O}_{y}$. As we always identify $R_{\chi}$ as a subgroup of $W_{\chi} \subset W$, thus $\sigma_{\mathcal{O}_{y}}^{\mathscr{X}}$ can be viewed as a representation of $R_{\chi}$ by restriction.

Conjecture 5.3. Let $\bar{G}$ be a saturated $n$-fold cover of a semisimple simply-connected group $G$. Let $\chi$ be a unitary unramified genuine character of $Z(\bar{T})$. Then for the natural correspondence (as discussed in $\$ 4.1$ )

$$
\operatorname{Irr}\left(R_{\chi}\right) \longrightarrow \Pi(\chi), \quad \sigma \mapsto \pi_{\sigma}
$$

one has

$$
\operatorname{dim} \mathrm{Wh}_{\psi}\left(\pi_{\sigma}\right)_{\mathcal{O}_{y}}=\left\langle\sigma, \sigma_{\mathcal{O}_{y}}^{\mathscr{X}}\right\rangle_{R_{\chi}}
$$

for every $W$-orbit $\mathcal{O}_{y}$, where $\left\langle\sigma_{1}, \sigma_{2}\right\rangle_{R_{\chi}}$ is the pairing of the two representations $\sigma_{1}, \sigma_{2}$ of $R_{\chi}$. In particular, $\operatorname{dim} \mathrm{Wh}_{\psi}\left(\pi_{\sigma}\right)=\left\langle\sigma, \sigma^{\mathscr{X}}\right\rangle_{R_{\chi}}$ for every $\sigma \in \operatorname{Irr}\left(R_{\chi}\right)$.

Note that the conjecture is trivially true for arbitrary $\bar{G}$ if $R_{\chi}=\{1\}$. This applies in particular to all Brylinski-Deligne covers of $\mathrm{GL}_{r}$, for which $R_{\chi}$ is always trivial. We also recall that if $G$ is simply-connected, then by Definition $2.1, \bar{G}$ is saturated if and only if the dual group $\bar{G}^{\vee}$ is of adjoint type, i.e., $Y_{Q, n}=Y_{Q, n}^{s c}$ in this case. 
Remark 5.4. Conjecture 5.3 is compatible with the decomposition

$$
I(\chi)=\bigoplus_{\sigma \in \operatorname{Irr}\left(R_{\chi}\right)} \pi_{\sigma}
$$

Indeed, one has

$$
\sum_{\sigma \in \operatorname{Irr}\left(R_{\chi}\right)}\left\langle\sigma, \sigma_{\mathcal{O}_{y}}^{\mathscr{X}}\right\rangle_{R_{\chi}}=\left\langle\mathbf{C}\left[R_{\chi}\right], \sigma_{\mathcal{O}_{y}}^{\mathscr{X}}\right\rangle_{R_{\chi}}=\left|\mathcal{O}_{y}\right|,
$$

which is equal to $\operatorname{dim} \mathrm{Wh}_{\psi}(I(\chi))_{\mathcal{O}_{y}}$.

Remark 5.5. Ginzburg proposed in [Gin18, Conjecture 1] that if $\pi$ is an irreducible unramified representation of $\bar{G}$ which is non-generic, then there exists a non-generic theta representation $\Theta$ of a Levi subgroup $\bar{M} \subset \bar{P} \subset \bar{G}$ such that $\pi \hookrightarrow \operatorname{Ind} \frac{\bar{G}}{P}(\Theta)$. On the other hand, Conjecture 5.3 implies that for simply-connected $G$, we have $\operatorname{dim} \mathrm{Wh}_{\psi}\left(\pi_{\chi}^{u n}\right)=$ $\left|\mathcal{O}_{\mathscr{X}}^{R_{\chi}}\right|$ (the number of $R_{\chi}$-orbits in $\mathscr{X}_{Q, n}$ ), since $\pi_{\chi}^{u n}=\pi_{\mathbb{1}}$. That is, Ginzburg's conjecture is vacuously true for such unramified representation $\pi_{\chi}^{u n}$. For a comparison with the case of regular unramified $\chi$, see [Gaob, Remark 7.4].

5.4. A formula for $\operatorname{dim} \mathrm{Wh}_{\psi}\left(\pi_{\sigma}\right)$. In this subsection, let $G$ be a general connected reductive group and let $\bar{G}$ be an $n$-fold cover, unless specified otherwise. Consider the decomposition

$$
I(\chi)=\bigoplus_{\sigma \in \operatorname{Irr}\left(R_{\chi}\right)} \pi_{\sigma}
$$

Let $\mathbb{w} \in W_{\chi}$. We have ${ }^{w} \chi \simeq \chi$, and thus an endomorphism

$$
\mathscr{A}(w, \chi)^{*}: \mathrm{Wh}_{\psi}(I(\chi)) \rightarrow \mathrm{Wh}_{\psi}(I(\chi))
$$

induced from $\mathscr{A}(w, \chi)=\gamma(w, \chi) \cdot A(w, \chi)$, see Lemma 4.1. In fact, if $\mathbb{w} \in R_{\chi}$, then the normalizing factor is nonzero, and thus $A(w, \chi)$ is already holomorphic for every $w \in R_{\chi}$. In any case, it follows from Corollary 4.7 that for $\mathbb{w} \in R_{\chi}$

$$
\mathscr{A}(w, \chi)^{*}=\bigoplus_{\sigma \in \operatorname{Irr}\left(R_{\chi}\right)} \sigma(\mathbb{w}) \cdot \operatorname{id}_{\mathrm{Wh}_{\psi}\left(\pi_{\sigma}\right)}
$$

and therefore the characteristic polynomial of $\mathscr{A}(w, \chi)^{*}$ is

$$
\begin{aligned}
\operatorname{det}\left(X \cdot \mathrm{id}-\mathscr{A}(w, \chi)^{*}\right) & =\operatorname{det}\left(X \cdot I_{\left|\mathscr{X}_{Q, n}\right|}-\mathscr{A}(w, \chi)^{*}\right) \\
& =\prod_{\sigma \in \operatorname{Irr}\left(R_{\chi}\right)}(X-\sigma(\mathbb{w}))^{\operatorname{dim} \mathrm{Wh}_{\psi}\left(\pi_{\sigma}\right)}
\end{aligned}
$$

For every $\mathbb{w} \in W$ and $\mathcal{O}_{y} \in \mathcal{O}_{\mathscr{X}}$, the restriction of $\mathscr{A}(w, \chi)^{*}: \mathrm{Wh}_{\psi}\left(I\left({ }^{w} \chi\right)\right) \rightarrow$ $\mathrm{Wh}_{\psi}(I(\chi))$ to $\mathrm{Wh}_{\psi}\left(I\left({ }^{w} \chi\right)\right)_{\mathcal{O}_{y}}$ gives a well-defined homomorphism

$$
\mathscr{A}(w, \chi)_{\mathcal{O}_{y}}^{*}: \mathrm{Wh}_{\psi}\left(I\left({ }^{w} \chi\right)\right)_{\mathcal{O}_{y}} \rightarrow \mathrm{Wh}_{\psi}(I(\chi))_{\mathcal{O}_{y}}
$$

represented by $\mathcal{S}_{\mathfrak{R}}(w, i(\chi))_{\mathcal{O}_{y}}$, see [Gaob, Proposition 4.4]. Moreover,

$$
\mathscr{A}(w, \chi)^{*}=\bigoplus_{\mathcal{O}_{y} \in \mathcal{O}_{\mathscr{X}}} \mathscr{A}(w, \chi)_{\mathcal{O}_{y}}^{*}
$$

where the sum is taken over all $W$-orbits in $\mathscr{X}_{Q, n}$. For $\mathbb{w} \in R_{\chi}$, this gives that

$$
\mathscr{A}(w, \chi)_{\mathcal{O}_{y}}^{*}=\bigoplus_{\sigma \in \operatorname{Irr}\left(R_{\chi}\right)} \sigma(\mathbb{w}) \cdot \operatorname{id}_{\mathrm{Wh}_{\psi}\left(\pi_{\sigma}\right)_{\mathcal{O}_{y}}} .
$$


For every $W$-orbit $\mathcal{O}_{y} \subset \mathscr{X}_{Q, n}$, consider the map

$$
\sigma_{\mathcal{O}_{y}}^{\mathrm{Wh}}: R_{\chi} \longrightarrow \mathrm{GL}\left(\mathrm{Wh}_{\psi}(I(\chi))_{\mathcal{O}_{y}}\right)
$$

given by

Also let

$$
\sigma_{\mathcal{O}_{y}}^{\mathrm{Wh}}(\mathbb{w}):=\mathscr{A}(w, \chi)_{\mathcal{O}_{y}}^{*} .
$$

$$
\sigma^{\mathrm{Wh}}: R_{\chi} \longrightarrow \mathrm{GL}\left(\mathrm{Wh}_{\psi}(I(\chi))\right)
$$

be the map given by

$$
\sigma^{\mathrm{Wh}}(\mathbb{w}):=\mathscr{A}(w, \chi)^{*} .
$$

It is then clear from (30) that

$$
\sigma^{\mathrm{Wh}}=\bigoplus_{\mathcal{O}_{y} \in \mathcal{O}_{\mathscr{X}}} \sigma_{\mathcal{O}_{y}}^{\mathrm{Wh}}
$$

Theorem 5.6. Let $\bar{G}$ be an $n$-fold cover of a connected reductive group $G$. Then for every $\mathcal{O}_{y} \in \mathcal{O}_{\mathscr{X}}$, the map $\sigma_{\mathcal{O}_{y}}^{\mathrm{Wh}}$ is a well-defined group homomorphism and

$$
\operatorname{dim} \mathrm{Wh}_{\psi}\left(\pi_{\sigma}\right)_{\mathcal{O}_{y}}=\left\langle\sigma, \sigma_{\mathcal{O}_{y}}^{\mathrm{Wh}}\right\rangle_{R_{\chi}}
$$

for every $\sigma \in \operatorname{Irr}\left(R_{\chi}\right)$. Hence, $\operatorname{dim} \mathrm{Wh}_{\psi}\left(\pi_{\sigma}\right)=\left\langle\sigma, \sigma^{\mathrm{Wh}}\right\rangle_{R_{\chi}}$.

Proof. For $\mathbb{w}, \mathbb{w}^{\prime} \in R_{\chi}$, we have

$$
\begin{aligned}
\sigma_{\mathcal{O}_{y}}^{\mathrm{Wh}}(\mathbb{w}) \circ \sigma_{\mathcal{O}_{y}}^{\mathrm{Wh}}\left(\mathbb{w}^{\prime}\right) & =\mathscr{A}(w, \chi)_{\mathcal{O}_{y}}^{*} \circ \mathscr{A}\left(w^{\prime}, \chi\right)_{\mathcal{O}_{y}}^{*} \\
& =\mathscr{A}(w, \chi)_{\mathcal{O}_{y}}^{*} \circ \mathscr{A}\left(w^{\prime},{ }^{w} \chi\right)_{\mathcal{O}_{y}}^{*} \\
& =\left(\mathscr{A}\left(w^{\prime},{ }^{w} \chi\right) \circ \mathscr{A}(w, \chi)\right)_{\mathcal{O}_{y}}^{*} \\
& =\mathscr{A}\left(w^{\prime} w, \chi\right)_{\mathcal{O}_{y}}^{*} \\
& =\sigma_{\mathcal{O}_{y}}^{\mathrm{Wh}}\left(\mathbb{w}^{\prime} \mathbb{w}\right)=\sigma_{\mathcal{O}_{y}}^{\mathrm{Wh}}\left(\mathbb{w}^{\prime} w^{\prime}\right),
\end{aligned}
$$

where the last equality follows from the fact that $R_{\chi}$ is abelian (see Theorem 4.6). This shows that $\sigma_{\mathcal{O}_{y}}^{\mathrm{Wh}}$ is a representation of $R_{\chi}$ on $\mathrm{Wh}_{\psi}(I(\chi))_{\mathcal{O}_{y}}$. Clearly, the equality in (31) gives

$$
\sigma_{\mathcal{O}_{y}}^{\mathrm{Wh}}=\bigoplus_{\sigma \in \operatorname{Irr}\left(R_{\chi}\right)} \operatorname{dim} \mathrm{Wh}_{\psi}\left(\pi_{\sigma}\right)_{\mathcal{O}_{y}} \cdot \sigma
$$

and thus it follows that

$$
\operatorname{dim} \mathrm{Wh}_{\psi}\left(\pi_{\sigma}\right)_{\mathcal{O}_{y}}=\left\langle\sigma, \sigma_{\mathcal{O}_{y}}^{\mathrm{Wh}}\right\rangle_{R_{\chi}} .
$$

This completes the proof.

If $\mathbb{w} \in R_{\chi}$, then $c_{\mathrm{gk}}(w, \chi)^{-1}=\gamma(w, \chi)$ (see Lemma 4.1) is actually holomorphic and nonzero at $\chi$. Denote by $\theta_{\sigma_{\mathcal{O}}}^{\mathrm{Wh}}$ the character of $\sigma_{\mathcal{O}_{y}}^{\mathrm{Wh}}$. For $\mathbb{w} \in R_{\chi}$, one has

$$
\theta_{\sigma_{\mathcal{O}_{y}}}(\mathbb{w})=\gamma(w, \chi) \cdot \operatorname{Tr}\left(A(w, \chi)_{\mathcal{O}_{y}}^{*}\right)
$$

and thus

$$
\operatorname{dim} \mathrm{Wh}_{\psi}\left(\pi_{\sigma}\right)_{\mathcal{O}_{y}}=\frac{\gamma(w, \chi)}{\left|R_{\chi}\right|} \cdot \sum_{\mathbb{w} \in R_{\chi}} \overline{\sigma(\mathbb{w})} \cdot \operatorname{Tr}\left(A(w, \chi)_{\mathcal{O}_{y}}^{*}\right) .
$$

Recall the permutation representation

$$
\sigma_{\mathcal{O}_{y}}^{\mathscr{X}}: W \longrightarrow \operatorname{Perm}\left(\mathcal{O}_{y}\right)
$$


associated to the $W$-orbit $\mathcal{O}_{y}$. Let $\theta_{\sigma_{\mathcal{O}_{y}}}$ be the character of $\sigma_{\mathcal{O}_{y}}^{\mathscr{X}}$. Then for every $\mathbb{w} \in W$, one has

$$
\theta_{\sigma_{\mathcal{O}}^{\mathscr{X}}}(\mathbb{w})=\left|\left(\mathcal{O}_{y}\right)^{\mathbb{w}}\right|
$$

where

$$
\left(\mathcal{O}_{y}\right)^{\mathbb{w}}=\left\{y \in \mathcal{O}_{y}: \mathbb{w}[y]=y\right\} .
$$

By restriction, we view both $\sigma^{\mathscr{X}}$ and $\sigma_{\mathcal{O}_{y}}^{\mathscr{X}}$ as representations of $R_{\chi}$. Conjecture 5.3 could be reformulated as follows.

Conjecture 5.7. Let $\bar{G}$ be a saturated $n$-fold cover of a semisimple simply-connected group $G$. Let $\chi$ be a unitary unramified genuine character of $Z(\bar{T})$. Then for every $W$-orbit $\mathcal{O}_{y}$, one has

$$
\sigma_{\mathcal{O}_{y}}^{\mathrm{Wh}} \simeq \sigma_{\mathcal{O}_{y}}^{\mathscr{X}}
$$

equivalently but more explicitly, for every $\mathbb{w} \in R_{\chi}$,

$$
\operatorname{Tr}\left(A(w, \chi)_{\mathcal{O}_{y}}^{*}\right)=\left|\left(\mathcal{O}_{y}\right)^{\mathbb{w}}\right| \cdot \gamma(w, \chi)^{-1}
$$

The equivalence between Conjecture 5.3 and Conjecture 5.7 follows from Theorem 5.6. The computation of $\operatorname{Tr}\left(A(w, \chi)_{\mathcal{O}_{y}}^{*}\right)$ is equivalent to that of $\operatorname{Tr}\left(\mathcal{S}_{\mathfrak{R}}(w, i(\chi))_{\mathcal{O}_{y}}\right)$ for any representative set $\mathfrak{R} \subset Y$ of $\mathscr{X}_{Q, n}$.

Remark 5.8. Conjecture 5.7 answers in a special case for the scattering matrix the analogous question raised in [GSS, §3.2] regarding the trace of a local coefficients matrix. We note that if ${ }^{w} \chi=\chi$, then both the local coefficients matrix and the scattering matrix associated to the operator $A(w, \chi)^{*}$ give invariants of the operator, albeit different. In this paper, it is the latter that is used and plays a crucial role in determining $\operatorname{dim} \mathrm{Wh}_{\psi}\left(\pi_{\sigma}\right)$. We hope that this phenomenon also helps justify our viewpoint in [GSS] that both the local coefficients matrix and the scattering matrix are important objects and should be studied together.

5.5. Double cover of $\mathrm{GSp}_{2 r}$. In this subsection, we apply Theorem 5.6 to the double cover of $\mathrm{GSp}_{2 r}$ and show that it recovers [Szp15, Corollary 6.6]. Meanwhile, we also show that the analogue of Conjecture 5.7 fails for such covers. This example shows that one can not extend the conjecture in a naive way to covers of a reductive group whose derived subgroup is simply-connected.

Let $\mathrm{GSp}_{2 r}$ be the group of similitudes of symplectic type, and let $\left(X, \Delta, Y, \Delta^{\vee}\right)$ be its root data given as follows. The character group $X \simeq \mathbf{Z}^{r+1}$ has a standard basis

$$
\left\{e_{i}^{*}: 1 \leq i \leq r\right\} \cup\left\{e_{0}^{*}\right\},
$$

where the simple roots are

$$
\Delta=\left\{e_{i}^{*}-e_{i+1}^{*}: 1 \leq i \leq r-1\right\} \cup\left\{2 e_{r}^{*}-e_{0}^{*}\right\} .
$$

The cocharacter group $Y \simeq \mathbf{Z}^{r+1}$ is given with a basis

$$
\left\{e_{i}: 1 \leq i \leq r\right\} \cup\left\{e_{0}\right\} .
$$

The simple coroots are

$$
\Delta^{\vee}=\left\{e_{i}-e_{i+1}: 1 \leq i \leq r-1\right\} \cup\left\{e_{r}\right\} .
$$

Write $\alpha_{i}=e_{i}^{*}-e_{i+1}^{*}, \alpha_{i}^{\vee}=e_{i}-e_{i+1}$ for $1 \leq i \leq r-1$, and also $\alpha_{r}=2 e_{r}^{*}-e_{0}^{*}, \alpha_{r}^{\vee}=e_{r}$. Consider the covering $\overline{\mathrm{GSp}}_{2 r}$ incarnated by $(D, \mathbb{1})$. We are interested in those $\overline{\mathrm{GSp}}_{2 r}$ whose restriction to $\mathrm{Sp}_{2 r}$ is the one with $Q\left(\alpha_{r}^{\vee}\right)=1$. That is, we assume

$$
Q\left(\alpha_{i}^{\vee}\right)=2 \text { for } 1 \leq i \leq r-1 \text {, and } Q\left(\alpha_{r}^{\vee}\right)=1 \text {. }
$$


Since $\Delta^{\vee} \cup\left\{e_{0}\right\}$ gives a basis for $Y$, to determine $Q$ it suffices to specify $Q\left(e_{0}\right)$. For $n=2$, we will obtain a double cover $\overline{\mathrm{GSp}}_{2 r}$ which restricts to the classical metaplectic double cover $\overline{\mathrm{Sp}}_{2 r}$. The number $Q\left(e_{0}\right) \in \mathbf{Z} / 2 \mathbf{Z}$ determines whether the similitude factor $F^{\times}$corresponding to the cocharacter $e_{0}$ splits into $\overline{\mathrm{GSp}}_{2 r}$ or not. To recover the classical double cover of $\mathrm{GSp}_{2 r}$ (see [Szp15]), we take $Q\left(e_{0}\right)$ to be an even number in this subsection.

In this case, one has

$$
Y_{Q, 2}=\left\{\sum_{i=1}^{r} k_{i} \alpha_{i}^{\vee}+k e_{0} \in Y: k_{i} \in \mathbf{Z} \text { for } 1 \leq i \leq r-1, k_{r}, k \in 2 \mathbf{Z}\right\} .
$$

The sublattice $Y_{Q, 2}^{s c}$ is spanned by $\left\{\alpha_{i, Q, 2}^{\vee}\right\}_{1 \leq i \leq r}$, i.e.,

$$
\left\{\alpha_{1}^{\vee}, \alpha_{2}^{\vee}, \ldots, \alpha_{r-1}^{\vee}, 2 \alpha_{r}^{\vee}\right\} .
$$

Regarding the dual group of the double cover $\overline{\mathrm{GSp}}_{2 r}$, one has

$$
\overline{\mathrm{GSp}}_{2 r}^{\vee}=\left\{\begin{array}{l}
\mathrm{GSp}_{2 r}(\mathbf{C}), \text { if } r \text { is odd; } \\
\mathrm{PGSp}_{2 r}(\mathbf{C}) \times \mathrm{GL}_{1}(\mathbf{C}), \text { if } r \text { is even. }
\end{array}\right.
$$

Thus, $\mathbf{H}$ is $\mathrm{GSp}_{2 r}$ (resp. $\left.\operatorname{Spin}_{2 r+1} \times \mathrm{GL}_{1}\right)$ if $r$ is odd (resp. even). Note that

$$
\mathscr{X}_{Q, 2}=Y / Y_{Q, 2}=\left\{0, e_{r}, e_{0}, e_{r}+e_{0}\right\},
$$

which is isomorphic to $\mathbf{Z} / 2 \mathbf{Z} \times \mathbf{Z} / 2 \mathbf{Z}$.

If $r$ is odd, then the torus $T_{Q, 2}$ of $\mathbf{H}$ acts transitively on all non-degenerate characters of the unipotent subgroup of the Borel subgroup of $\mathbf{H}$. Thus, $R_{\underline{\chi}}=\{1\}$ for every unramified unitary character $\underline{\chi}$ (see [Li92, Lemma 2.5]). Therefore, the R-group $R_{\chi}$ for $\overline{\mathrm{GSp}}_{2 r}$ with $r$ odd is trivial for every unitary unramified genuine character $\chi$.

We assume that $r$ is even. For every $\alpha \in \Phi$, recall that we have the notation (see $§ 3.2$ )

$$
\chi_{\alpha}:=\underline{\chi}_{\alpha}(\varpi)=\chi\left(\bar{h}_{\alpha}\left(\varpi^{n_{\alpha}}\right)\right) .
$$

It follows from [Key82b, Page 399] that the only nontrivial $R_{\chi}$ is $\{1, \mathbb{w}\} \simeq \mathbf{Z} / 2 \mathbf{Z}$ which is generated by

$$
\mathbb{W}:=\mathbb{w}_{\alpha_{1}} \mathbb{W}_{\alpha_{3}} \ldots \mathbb{W}_{\alpha_{r-1}},
$$

with the character $\chi$ satisfying

$$
\chi_{\alpha_{i}}=-1 \text { for all } i=2 k-1,1 \leq k \leq r / 2 .
$$

Proposition 5.9. Assume $r$ even and $\chi$ is an unramified character satisfying the above condition. Then as a representation of $R_{\chi}$,

$$
\sigma^{\mathrm{Wh}} \simeq 2 \cdot \mathbb{1} \oplus 2 \cdot \varepsilon
$$

where $\varepsilon$ denotes the non-trivial character of $R_{\chi}$.

Proof. It suffices to compute the trace of $\sigma^{\mathrm{Wh}}$. It is easy to see that for every $y \in \mathscr{X}_{Q, 2}$ and $\mathbb{w}_{\alpha_{i}}, i=1,3, \ldots, r-1$, one has

$$
\mathbb{w}_{\alpha_{i}}[y]=y \in \mathscr{X}_{Q, 2} .
$$

Thus, by [GSS, Proposition 4.12], the $(y, y)$-entry of $A(w, y)^{*}$ is given by

$$
\tau\left(w, \chi, \mathbf{s}_{y}, \mathbf{s}_{y}\right)=\gamma(w, \chi)^{-1} \cdot \prod_{i=1,3, \ldots, r-1} \chi_{\alpha_{i}}^{\left\langle y, \alpha_{i}\right\rangle}=\gamma(w, \chi)^{-1} \cdot(-1)^{\left\langle y, \alpha_{i}\right\rangle} .
$$


We see

$$
\gamma(w, \chi) \cdot \tau\left(w, \chi, \mathbf{s}_{y}, \mathbf{s}_{y}\right)= \begin{cases}1 & \text { if } y=0, \\ -1 & \text { if } y=e_{r}, \\ 1 & \text { if } y=e_{0}, \\ -1 & \text { if } y=e_{r}+e_{0} .\end{cases}
$$

This shows that $\operatorname{Tr}\left(\sigma^{\mathrm{Wh}}\right)(w)=0$. Thus, $\sigma^{\mathrm{Wh}}=2 \cdot \mathbb{1} \oplus 2 \cdot \varepsilon$, as claimed.

Theorem 5.10 ([Szp15, Corollary 6.6]). If $r$ is odd, then every unitary unramified genuine principal series $I(\chi)$ for the double cover $\overline{\mathrm{GSp}}_{2 r}$ is irreducible. If $r$ is even, then the only reducibility of $I(\chi)$ occurs when $R_{\chi} \simeq \mathbf{Z} / 2 \mathbf{Z}$; in this case, $I(\chi)=\pi_{\chi}^{u n} \oplus \pi_{\varepsilon}$, and

$$
\operatorname{dim} \mathrm{Wh}_{\psi}\left(\pi_{\chi}^{u n}\right)=\operatorname{dim} \mathrm{Wh}_{\psi}\left(\pi_{\varepsilon}\right)=2 .
$$

Proof. We only need to show the last two equalities, which follow from combining Theorem 5.6 and Proposition 5.9.

Remark 5.11. It follows from the proof of Proposition 5.9 that $\mathbb{w}[y]=y$ for every $y \in \mathscr{X}_{Q, 2}$. Thus,

$$
\sigma^{\mathscr{X}}=4 \cdot \mathbb{1}
$$

in particular, it is not isomorphic to $\sigma^{\mathrm{Wh}}$. We see that the (naive) analogue of Conjecture 5.7 fails for such $\overline{\mathrm{GSp}}_{2 r}$.

\section{ON THE DIMENSION OF $\mathrm{Wh}_{\psi}\left(\pi_{\chi}^{u n}\right)$}

6.1. Lower bound for $\operatorname{dim} \mathrm{Wh}_{\psi}\left(\pi_{\chi}^{u n}\right)$. In this subsection, we will prove Conjecture 5.7 for $\pi_{\chi}^{u n}$ in a special case, which gives a lower bound of $\operatorname{dim} \mathrm{Wh}_{\psi}\left(\pi_{\chi}^{u n}\right)$.

Let $\bar{G}$ be an $n$-fold covering group of a connected reductive group $G$. Assume that $\bar{G}$ is not of metaplectic-type (see Definition 2.1). We call $z \in Y$ an exceptional point (see [GSS, Definition 5.1]) if

for every $\alpha \in \Delta$, that is,

$$
\left\langle z_{\rho}, \alpha\right\rangle=-n_{\alpha}
$$

$$
\mathbb{w}_{\alpha}[z]=z+\alpha_{Q, n}^{\vee} \text { for every } \alpha \in \Delta .
$$

Note that the definition here is the same as [GSS, Definition 5.1], since we have assumed that $G$ is not of metaplectic type.

For $\bar{G}$ not of metaplectic type, denote by $Y_{n}^{\text {exc }} \subset Y$ the set of exceptional points. Let

$$
f: Y \rightarrow \mathscr{X}_{Q, n}
$$

be the quotient map, and denote

$$
\mathscr{X}_{Q, n}^{\mathrm{exc}}:=f\left(Y_{n}^{\mathrm{exc}}\right) .
$$

If $y \in Y$ is exceptional, then $y \in f^{-1}\left(\mathscr{X}_{Q, n}^{W}\right)$; that is,

$$
\mathscr{X}_{Q, n}^{\mathrm{exc}} \subset\left(\mathscr{X}_{Q, n}\right)^{W} \text {. }
$$

Denoting

$$
\rho_{Q, n}:=\frac{1}{2} \sum_{\alpha>0} \alpha_{Q, n}^{\vee} \in Y \otimes \mathbf{Q},
$$

we always have

$$
\left(\left\{\rho-\rho_{Q, n}\right\} \cap Y\right) \subseteq Y_{n}^{\text {exc }} .
$$

If $G$ is a semisimple group and $\bar{G}$ is not of metaplectic type, then (see [GSS, Lemma 5.2])

$$
Y_{n}^{\text {exc }}=\left\{\rho-\rho_{Q, n}\right\} \cap Y ;
$$


that is, $Y_{n}^{\text {exc }}$ contains the unique element $\rho-\rho_{Q, n}$ if it lies in $Y$. It is also determined explicitly in [GSS, §6- $\S 7$ ] the dependence of $Y_{n}^{\text {exc }}$ on $\bar{G}$ for covers of simply-connected groups.

Theorem 6.1. Let $\bar{G}$ be an $n$-fold cover of a connected reductive group $G$. Assume that $\bar{G}$ is not of metaplectic type. Then for every $z \in \mathscr{X}_{Q, n}^{\operatorname{exc}}$ and $\mathbb{w} \in R_{\chi}$, one has $\mathcal{S}_{\mathfrak{R}}(w, i(\chi))_{\mathcal{O}_{z}}=\gamma(w, \chi)^{-1}$. Therefore,

$$
\sigma_{\mathcal{O}_{z}}^{\mathrm{Wh}}=\sigma_{\mathcal{O}_{z}}^{\mathscr{X}}=\mathbb{1}_{R_{\chi}},
$$

and thus

$$
\operatorname{dim} \mathrm{Wh}_{\psi}\left(\pi_{\chi}^{u n}\right) \geq\left|\mathscr{X}_{Q, n}^{\mathrm{exc}}\right|
$$

In particular, if $\rho-\rho_{Q, n} \in Y$, then $\pi_{\chi}^{u n}$ is generic. Moreover, Conjecture 5.7 holds for such $\mathcal{O}_{z}$.

Proof. Since $\mathcal{O}_{z}=\{z\}$, we have

$$
\mathcal{S}_{\mathfrak{R}}(w, i(\chi))_{\mathcal{O}_{z}}=\tau\left(w, \chi, \mathbf{s}_{z}, \mathbf{s}_{z}\right) .
$$

First, we note that by (25) and the fact that $\chi$ is fixed by $\mathbb{w} \in R_{\chi}$, the entry $\tau\left(w, \chi, \mathbf{s}_{z}, \mathbf{s}_{z}\right)$ is independent of the representative for $z \in \mathscr{X}_{Q, n}^{\mathrm{exc}}$. It follows from [GSS, Proposition 4.12] (as $\bar{G}$ is not of metaplectic type) that

$$
\tau\left(w, \chi, \mathbf{s}_{z}, \mathbf{s}_{z}\right)=\gamma(w, \chi)^{-1}
$$

for every $z \in \mathscr{X}_{Q, n}^{\text {exc }}$, and Conjecture 5.7 holds for such $\mathcal{O}_{z}$. In fact, $\sigma_{\mathcal{O}_{z}}^{\mathrm{Wh}}=\sigma_{\mathcal{O}_{z}}^{\mathscr{X}}=\mathbb{1}_{R_{\chi}}$ and thus

$$
\operatorname{dim} \mathrm{Wh}_{\psi}\left(\pi_{\chi}^{u n}\right)_{\mathcal{O}_{z}}=\left\langle\mathbb{1}, \sigma_{\mathcal{O}_{z}}^{\mathrm{Wh}}\right\rangle_{R_{\chi}}=1
$$

Therefore, $\operatorname{dim} \mathrm{Wh}_{\psi}\left(\pi_{\chi}^{u n}\right) \geq\left|\mathscr{X}_{Q, n}^{\mathrm{exc}}\right|$. This completes the proof.

For covers of a semisimple and simply-connected group $G$, it follows from [GSS, Theorem 6.3] that

$$
0 \leq\left|\mathscr{X}_{Q, n}^{\mathrm{exc}}\right| \leq\left|\left(\mathscr{X}_{Q, n}\right)^{W}\right| \leq 1
$$

In fact, in [GSS, $\S 7$, we determined explicitly the size of the two sets $\mathscr{X}_{Q, n}^{\mathrm{exc}}$ and $\left(\mathscr{X}_{Q, n}\right)^{W}$. On the other hand, if $G$ is semisimple but not simply-connected, then it is possible to have

$$
\left|\mathscr{X}_{Q, n}^{\mathrm{exc}}\right| \leq 1<\left|\left(\mathscr{X}_{Q, n}\right)^{W}\right|
$$

See [GSS] for details.

We note that the equality $\sigma_{\mathcal{O}_{z}}^{\text {Wh }}=\sigma_{\mathcal{O}_{z}}^{\mathscr{X}}$ in Theorem 6.1 might fail for $z \in\left(\mathscr{X}_{Q, n}\right)^{W}-\mathscr{X}_{Q, n}^{\text {exc }}$ for covers of a semisimple group: we will consider such an example from $n$-fold covers of $\mathrm{SO}_{3}$ in the next section. This example shows that the naive analogue of Conjecture 5.3 does not hold for general semisimple groups.

Remark 6.2. If $G$ is almost simple and $\bar{G}$ is of metaplectic type, then it follows from the discussion after Definition 2.1 that $\bar{G}=\overline{\mathrm{Sp}}_{2 r}$ with $n_{\alpha} \equiv 2(\bmod 4)$. Moreover, Example 4.11 shows that $R_{\chi}=\{1\}$ in this case, and thus the (in-)equalities $\operatorname{dim} \mathrm{Wh}_{\psi}\left(\pi_{\chi}^{u n}\right)=$ $\left|\mathscr{X}_{Q, n}\right| \geq\left|\mathscr{X}_{Q, n}^{\mathrm{exc}}\right|$ hold trivially. 
6.2. Unramified Whittaker function. We note that Theorem 6.1 applied to the case $n=1$ shows that $\pi_{\chi}^{u n}$ is the only generic constituent of $I(\chi)$ for the linear algebraic group $G$. This fact also follows from the Casselman-Shalika formula [CS80]. Motivated by this, for covering groups, we consider in this subsection the relation between $\operatorname{dim} \mathrm{Wh}_{\psi}\left(\pi_{\chi}^{u n}\right)$ and the unramified Whittaker function, which is also the approach taken in [Gin18] but for a general unramified genuine character.

First, by restriction, one obtains a surjection of vector spaces

$$
h^{u n}: \mathrm{Wh}_{\psi}(I(\chi)) \rightarrow \mathrm{Wh}_{\psi}\left(\pi_{\chi}^{u n}\right) .
$$

Let $f^{0} \in \pi_{\chi}^{u n} \subset I(\chi)$ be the normalized unramified function. For $\mathbf{c} \in \operatorname{Ftn}(i(\chi))$, let $\lambda_{\mathbf{c}} \in \mathrm{Wh}_{\psi}(I(\chi))$ be the associated Whittaker functional, also viewed as an element in $\mathrm{Wh}_{\psi}\left(\pi_{\chi}^{u n}\right)$; that is, we may still use $\lambda_{\mathbf{c}}$ for $h^{u n}\left(\lambda_{\mathbf{c}}\right)$ if no confusion arises. The unramified Whittaker function on $\bar{G}$ associated with $\mathbf{c}$ is given by

$$
\mathcal{W}_{\mathbf{c}}(\bar{g})=\lambda_{\mathbf{c}}\left(\pi_{\chi}^{u n}(\bar{g}) f^{0}\right)=\lambda_{\mathbf{c}}\left(I(\chi)(\bar{g}) f^{0}\right)
$$

We have a decomposition $\bar{G}=\bar{B} K=U \bar{T} K$ and

$$
\mathcal{W}_{\mathbf{c}}(u \bar{t} k)=\psi(u) \cdot \mathcal{W}_{\mathbf{c}}(\bar{t}) \text { for } u \in U, \bar{t} \in \bar{T}, k \in K .
$$

Thus, the value of $\mathcal{W}_{\mathbf{c}}$ is determined by its restriction to $\bar{T}$. If $\mathbf{c}=\mathbf{c}_{\gamma}$ for $\gamma \in \bar{T}$, then we denote

$$
\mathcal{W}_{\gamma}:=\mathcal{W}_{\mathbf{c}_{\gamma}}
$$

Recall that $\bar{t} \in \bar{T}$ is called dominant if

$$
\bar{t} \cdot(U \cap K) \cdot \bar{t}^{-1} \subset K .
$$

Let

$$
Y^{+}=\{y \in Y:\langle y, \alpha\rangle \geq 0 \text { for all } \alpha \in \Delta\} .
$$

Then an element $\mathbf{s}_{y} \in \bar{T}$ is dominant if and only if $y \in Y^{+}$. The following result regarding $\mathcal{W}_{\gamma}(\bar{t})$ is shown in [KP84, Pat87, CO13] for coverings of $\mathrm{GL}_{r}$. For a general covering group, the idea is the same; it is implicit in [McN16] and explicated in [Gaoa].

Proposition 6.3. One has $\mathcal{W}_{\gamma}(\bar{t})=0$ unless $\bar{t} \in \bar{T}$ is dominant. Moreover, for dominant $\bar{t}$

$$
\mathcal{W}_{\gamma}(\bar{t})=\delta_{B}^{1 / 2}(\bar{t}) \cdot \sum_{\mathrm{w} \in W} c_{\mathrm{gk}}\left(w_{G} w^{-1}, \chi\right) \cdot \tau\left(w,{ }^{-1} \chi, \gamma, w_{G} \cdot \bar{t} \cdot w_{G}^{-1}\right)
$$

where $\delta_{B}$ is the modular character of $B$.

It follows that for $z \in Y$ and $y \in Y$,

$$
\mathcal{W}_{\mathbf{s}_{z}}\left(\mathbf{s}_{y}\right)= \begin{cases}\delta_{B}^{1 / 2}\left(\mathbf{s}_{y}\right) \cdot \sum_{\mathbb{w} \in W} c_{\mathrm{gk}}\left(w_{G} w^{-1}, \chi\right) \cdot \tau\left(w, w^{-1} \chi, \mathbf{s}_{z}, w_{G} \cdot \mathbf{s}_{y} \cdot w_{G}^{-1}\right) & \text { if } y \in Y^{+}, \\ 0 & \text { otherwise. }\end{cases}
$$

We define for every $\gamma, \bar{t} \in \bar{T}$,

$$
\mathcal{W}_{\gamma}^{*}(\bar{t}):=\delta_{B}^{1 / 2}(\bar{t})^{-1} \cdot \sum_{\mathrm{w} \in W} c_{\mathrm{gk}}\left(w_{G} w^{-1}, \chi\right) \cdot \tau\left(w,{ }^{w^{-1}} \chi, \gamma, \bar{t}\right)
$$

We emphasize that here $\bar{t}$ is not required to be dominant. In particular,

$$
\mathcal{W}_{\mathbf{s}_{z}}^{*}\left(\mathbf{s}_{y}\right)=\delta_{B}^{1 / 2}\left(\mathbf{s}_{y}\right)^{-1} \cdot \sum_{\mathbf{w} \in W} c_{\mathrm{gk}}\left(w_{G} w^{-1}, \chi\right) \cdot \tau\left(w,{ }^{-1} \chi, \mathbf{s}_{z}, \mathbf{s}_{y}\right)
$$

for every $z, y \in Y$. One can extend by linearity and define $\mathcal{W}_{\mathbf{c}}^{*}(\bar{t})$ for every $\mathbf{c} \in \operatorname{Ftn}(i(\chi))$. If $w_{G}^{-1} \bar{t} w_{G}$ is dominant, then

$$
\mathcal{W}_{\mathbf{c}}^{*}(\bar{t})=\mathcal{W}_{\mathbf{c}}\left(w_{G}^{-1} \bar{t} w_{G}\right)
$$


We denote

$$
\mathcal{W}^{*}:=\left\{\mathcal{W}_{\mathbf{c}}^{*}: \mathbf{c} \in \operatorname{Ftn}(i(\chi))\right\} .
$$

If $\mathcal{W}_{\mathbf{c}}^{*}=0$ as a function of $\bar{T}$, then $\mathcal{W}_{\mathbf{c}}=0$ and it follows that $\lambda_{\mathbf{c}}(v)=0$ for every $v \in \pi_{\chi}^{u n}$ as the unramified vector $f^{0}$ generates $\pi_{\chi}^{u n}$. Thus, one has an injection of vector spaces

$$
\mathrm{Wh}_{\psi}\left(\pi_{\chi}^{u n}\right) \longleftrightarrow \mathcal{W}^{*}
$$

given by

$$
\lambda_{\mathbf{c}} \mapsto \mathcal{W}_{\mathbf{c}}^{*}
$$

For $z \in \mathscr{X}_{Q, n}$, let $\mathcal{W}_{\mathcal{O}_{z}}^{*} \subset \mathcal{W}^{*}$ be the subspace spanned by $\left\{\mathcal{W}_{\mathbf{s}_{z^{\prime}}}^{*}: z^{\prime} \in \mathfrak{R}_{z}\right\}$, where $\mathfrak{R}_{z} \subset Y$ is a set of representatives of $\mathcal{O}_{z}$. Note that $\mathcal{W}_{\mathbf{s}_{z^{\prime}}}^{*}$ depends on the representative $z^{\prime}$; however, the space $\mathcal{W}_{\mathcal{O}_{z}}^{*}$ depends only on $\mathcal{O}_{z}$. From the restriction of (33), we obtain

$$
\mathrm{Wh}_{\psi}\left(\pi_{\chi}^{u n}\right)_{\mathcal{O}_{z}} \longleftrightarrow \mathcal{W}_{\mathcal{O}_{z}}^{*}
$$

Let $\mathbf{C}^{\left|\mathcal{O}_{z}\right|}$ be the $\left|\mathcal{O}_{z}\right|$-dimensional complex vector space. Endow the space $\mathbf{C}^{\left|\mathcal{O}_{z}\right|}$ with the coordinates indexed by $\mathfrak{R}_{z}$. Thus we write $\left(c_{y}\right)_{y \in \Re_{z}}$ for a general vector in $\mathbf{C}^{\left|\mathcal{O}_{z}\right|}$. Depending on $\mathfrak{R}_{z}$, there is an evaluation map

$$
\nu_{\mathfrak{R}_{z}}: \mathcal{W}_{\mathcal{O}_{z}}^{*} \longrightarrow \mathbf{C}^{\left|\mathcal{O}_{z}\right|}
$$

with the $y$-th coordinate of $\nu_{\mathfrak{R}_{z}}\left(\mathcal{W}_{\mathbf{s}_{z^{\prime}}}\right), y, z^{\prime} \in \mathfrak{R}_{z}$ given by

$$
\nu_{\mathfrak{R}_{z}}\left(\mathcal{W}_{\mathbf{s}_{z^{\prime}}}^{*}\right)_{y}=\mathcal{W}_{\mathbf{s}_{z^{\prime}}}^{*}\left(\mathbf{s}_{y}\right) \text {; }
$$

that is,

$$
\nu_{\mathfrak{R}_{z}}\left(\mathcal{W}_{\mathbf{s}_{z^{\prime}}}^{*}\right)=\left(\mathcal{W}_{\mathbf{s}_{z^{\prime}}}^{*}\left(\mathbf{s}_{y}\right)\right)_{y \in \Re_{z}} \in \mathbf{C}^{\left|\mathcal{O}_{z}\right|}
$$

If $\mathfrak{R} \subset Y$ is a set of representatives for $\mathscr{X}_{Q, n}$, then we have a unique subset $\mathfrak{R}_{z} \subset \mathfrak{R}$ representing $\mathcal{O}_{z}$. By combining all the $\nu_{\mathfrak{R}_{z}}$, one obtains an evaluation map

$$
\nu: \mathcal{W}^{*} \longrightarrow \mathbf{C}^{\left|\mathscr{X}_{Q, n}\right|}
$$

which depends on the chosen $\mathfrak{R}$. In particular, for a general $\mathbf{c} \in \operatorname{Ftn}(i(\chi))$, we have

$$
\nu\left(\mathcal{W}_{\mathbf{c}}^{*}\right)_{y}=\mathcal{W}_{\mathbf{c}}^{*}\left(\mathbf{s}_{y}\right) \text {. }
$$

For every $\mathcal{O}_{z} \in \mathcal{O}_{\mathscr{X}}$, composing (34) with $\nu_{\mathfrak{R}_{z}}$ gives a vector space homomorphism

$$
\nu_{\mathfrak{R}_{z}}^{\chi}: \mathrm{Wh}_{\psi}\left(\pi_{\chi}^{u n}\right)_{\mathcal{O}_{z}} \longrightarrow \mathbf{C}^{\left|\mathcal{O}_{z}\right|}
$$

Similarly, we have

$$
\nu^{\chi}: \mathrm{Wh}_{\psi}\left(\pi_{\chi}^{u n}\right) \longrightarrow \mathbf{C}^{\left|\mathscr{X}_{Q, n}\right|}
$$

with

$$
\nu^{\chi}=\bigoplus_{\mathcal{O}_{z} \in \mathcal{O}_{\mathscr{X}}} \nu_{\mathfrak{R}_{z}}^{\chi}
$$

Conjecture 6.4. For every $W$-orbit $\mathcal{O}_{z}$ and every choice of $\mathfrak{R}_{z}$, the homomorphism $\nu_{\mathfrak{R}_{z}}^{\chi}: \mathrm{Wh}_{\psi}\left(\pi_{\chi}^{u n}\right)_{\mathcal{O}_{z}} \rightarrow \mathbf{C}^{\left|\mathcal{O}_{z}\right|}$ is injective. Thus, $\operatorname{dim} \mathrm{Wh}_{\psi}\left(\pi_{\chi}^{u n}\right)_{\mathcal{O}_{z}}=\operatorname{rank}\left(\nu_{\mathfrak{R}_{z}}^{\chi}\right)$ and

$$
\operatorname{dim} \mathrm{Wh}_{\psi}\left(\pi_{\chi}^{u n}\right)=\sum_{\mathcal{O}_{z} \in \mathcal{O}_{\mathscr{X}}} \operatorname{rank}\left(\nu_{\mathfrak{R}_{z}}^{\chi}\right)
$$


For application purpose (i.e., to determine $\operatorname{dim} \mathrm{Wh}_{\psi}\left(\pi_{\chi}^{u n}\right)_{\mathcal{O}_{z}}$ ), it is sufficient to consider one $\mathfrak{R}_{z}$. More precisely, for low-rank groups, or low-degree covering groups, one can verify the injectivity in Conjecture 6.4 for a particular $\mathfrak{R}_{z}$ and compute the above $\operatorname{rank}\left(\nu_{\mathfrak{R}_{z}}^{\chi}\right)$ explicitly.

Theorem 6.5. Let $\bar{G}$ be an $n$-fold cover of a linear algebraic group $G$, which is not of metaplectic type (see Definition 2.1). For every $z \in Y_{n}^{\text {exc }}$, taking $\mathfrak{R}_{z}=\{z\}$, we have

$$
\operatorname{rank}\left(\nu_{\mathfrak{R}_{z}}^{\chi}\right)=1 \text {. }
$$

Hence, $\operatorname{dim} \mathrm{Wh}_{\psi}\left(\pi_{\chi}^{u n}\right) \geq\left|\mathscr{X}_{Q, n}^{\mathrm{exc}}\right|$.

Proof. If $z \in Y_{n}^{\text {exc }}$, then $-z$ is dominant and it follows from [GSS, Theorem 5.6] that we have a Casselman-Shalika formula for $\bar{G}$ which reads

$$
\mathcal{W}_{\mathbf{s}_{z}}^{*}\left(\mathbf{s}_{z}\right)=\delta_{B}^{1 / 2}\left(\mathbf{s}_{z}\right)^{-1} \cdot \prod_{\alpha>0}\left(1-q^{-1} \chi_{\alpha}\right)
$$

Since $\chi$ is unitary, we see that

$$
\nu_{\mathfrak{R}_{z}}^{\chi}\left(\lambda_{\mathbf{s}_{z}}\right)=\mathcal{W}_{\mathbf{s}_{z}}^{*}\left(\mathbf{s}_{z}\right) \neq 0 .
$$

This shows that $\nu_{\mathfrak{R}_{z}}^{\chi}$ is an isomorphism between the one-dimensional vector spaces. The result follows.

Theorem 6.5 is compatible with Theorem 6.1, though the approach in the former highlights the role of unramified Whittaker functions.

\section{Covers OF SYMPleCtiC GROUPS}

The goal of this section is to show that Conjecture 5.7 (equivalently Conjecture 5.3) holds for $\overline{\mathrm{Sp}}_{2 r}^{(n)}, r \geq 1$ and $\overline{\mathrm{SL}}_{3}^{(2)}$. Recall that for every $\alpha \in \Phi$ we denote

$$
\chi_{\alpha}:=\underline{\chi}_{\alpha}(\varpi)=\chi\left(\bar{h}_{\alpha}\left(\varpi^{n_{\alpha}}\right)\right) .
$$

7.1. Covers of $\mathrm{Sp}_{2 r}, r \geq 1$. Consider the Dynkin diagram for the simple coroots of $\mathrm{Sp}_{2 r}$ :

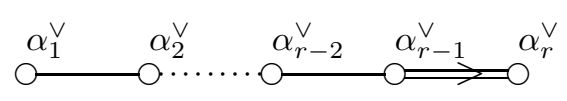

Let $Y=Y^{\mathrm{sc}}=\left\langle\alpha_{1}^{\vee}, \alpha_{2}^{\vee}, \ldots, \alpha_{r-1}^{\vee}, \alpha_{r}^{\vee}\right\rangle$ be the cocharacter lattice of $\mathrm{Sp}_{2 r}$, where $\alpha_{r}^{\vee}$ is the short coroot as shown in the above diagram. For simplicity, let $Q$ be the Weyl-invariant quadratic form on $Y$ such that $Q\left(\alpha_{r}^{\vee}\right)=1$. The bilinear form $B_{Q}$ is given by

$$
B_{Q}\left(\alpha_{i}^{\vee}, \alpha_{j}^{\vee}\right)= \begin{cases}2 & \text { if } i=j=r, \\ 4 & \text { if } 1 \leq i=j \leq r-1, \\ -2 & \text { if } j=i+1, \\ 0 & \text { if } \alpha_{i}^{\vee}, \alpha_{j}^{\vee} \text { are not adjacent. }\end{cases}
$$

Let $\bar{G}:=\overline{\mathrm{Sp}}_{2 r}^{(n)}$ be the $n$-fold cover of $\mathrm{Sp}_{2 r}$. We have

$$
\bar{G}^{\vee}= \begin{cases}\mathrm{Sp}_{2 r} & \text { if } n \text { is even; } \\ \mathrm{SO}_{2 r+1} & \text { if } n \text { is odd. }\end{cases}
$$

By Corollary 4.10, one has $R_{\chi}=\{1\}$ if $n$ even. For odd $n$, it is clear that $n_{\alpha_{i}}=n$ for all $\alpha_{i} \in \Delta$ and

$$
Y_{Q, n}=Y_{Q, n}^{s c}=n Y
$$


Following notations in [Bou02, Page 267], we consider the map

$$
\bigoplus_{i=1}^{r} \mathbf{Z} \alpha_{i}^{\vee} \rightarrow \bigoplus_{i=1}^{r} \mathbf{Z} e_{i}
$$

given by

$$
\left(x_{1}, x_{2}, x_{3} \ldots, x_{r}\right) \mapsto\left(x_{1}, x_{2}-x_{1}, x_{3}-x_{2}, \ldots, x_{r-1}-x_{r-2}, x_{r}-x_{r-1}\right),
$$

which is an isomorphism. The Weyl group is $W=S_{r} \rtimes(\mathbf{Z} / 2 \mathbf{Z})^{r}$, where $S_{r}$ is the permutation group on $\bigoplus_{i} \mathbf{Z} e_{i}$ and each $(\mathbf{Z} / 2 \mathbf{Z})_{i}$ acts by $e_{i} \mapsto \pm e_{i}$. In particular, $\mathbb{w}_{\alpha_{i}}, 1 \leq$ $i \leq r-1$, acts on $\left(y_{1}, y_{2}, \ldots, y_{r}\right) \in \bigoplus_{i} \mathbf{Z} e_{i}$ by exchanging $y_{i}$ and $y_{i+1}$, while $\mathbb{w}_{\alpha_{r}}$ acts by $(-1)$ on $\mathbf{Z} e_{r}$.

For odd $n$, it follows from Proposition 4.4, Proposition 4.5 and [Key82b, §3] that the only possible non-trivial $R$-group (up to isomorphism) for $\bar{G}$ is

$$
R_{\chi}=\left\{1, \mathbb{w}_{\alpha_{r}}\right\}
$$

where $\chi$ is the unramified genuine character of $Z(\bar{T}) \subset \bar{G}$ such that

- $\underline{\chi}_{\alpha_{i}}$ is any unitary unramified linear character for all $1 \leq i \leq r-1$, and

- furthermore

$$
\underline{\chi}_{\alpha_{r}}^{2}=\mathbb{1} \text { and } \underline{\chi}_{\alpha_{r}} \neq \mathbb{1}
$$

In particular, $\chi_{\alpha_{r}}=-1$. Denote by $\varepsilon$ the non-trivial character of $R_{\chi}$. We have a decomposition

$$
I(\chi)=\pi_{\chi}^{u n} \oplus \pi_{\varepsilon}
$$

where $\pi_{\varepsilon}$ is non-isomorphic to $\pi_{\chi}^{u n}=\pi_{\mathbb{1}}$.

Theorem 7.1. For odd $n$ and $\chi$ as above, Conjecture 5.7 holds for ${\overline{\mathrm{Sp}_{2 r}}}_{2 r}^{n}$; in this case,

$$
\operatorname{dim} \mathrm{Wh}_{\psi}\left(\pi_{\chi}^{u n}\right)=\frac{n^{r}+n^{r-1}}{2}, \quad \operatorname{dim} \mathrm{Wh}_{\psi}\left(\pi_{\varepsilon}\right)=\frac{n^{r}-n^{r-1}}{2} .
$$

Proof. For $n$ odd

$$
\mathscr{X}_{Q, n}=Y / Y_{Q, n} \simeq(\mathbf{Z} / n \mathbf{Z})^{r} .
$$

For every $W$-orbit $\mathcal{O}_{y} \subset \mathscr{X}_{Q, n}$, we will compute and check explicitly that

$$
\operatorname{Tr}\left(A\left(w_{\alpha_{r}}, \chi\right)_{\mathcal{O}_{y}}^{*}\right)=\left|\left(\mathcal{O}_{y}\right)^{\mathbb{w}_{\alpha_{r}}}\right| \cdot \gamma\left(w_{\alpha_{r}}, \chi\right)^{-1}
$$

One has a decomposition

$$
\mathcal{O}_{y}=\bigsqcup_{i \in I} \mathcal{O}_{z_{i}}^{R_{\chi}}
$$

of $\mathcal{O}_{y}$ into $R_{\chi}$-orbits, where

$$
\mathcal{O}_{z}^{R_{\chi}}=\{z\} \text { or } \mathcal{O}_{z}^{R_{\chi}}=\left\{z, \mathbb{w}_{\alpha_{r}}[z]\right\} .
$$

To show (35), it suffices to prove that for every $R_{\chi}$-orbit $\mathcal{O}_{z}^{R_{\chi}} \subset \mathscr{X}_{Q, n}$, one has

$$
\sum_{z^{\prime} \in \mathcal{O}_{z}^{R_{\chi}}} \tau\left(w_{\alpha_{r}}, \chi, \mathbf{s}_{z^{\prime}}, \mathbf{s}_{z^{\prime}}\right)=\left|\left(\mathcal{O}_{z}^{R_{\chi}}\right)^{\mathrm{w}_{\alpha_{r}}}\right| \cdot \gamma\left(w_{\alpha_{r}}, \chi\right)^{-1} .
$$

First, if $\mathcal{O}_{z}^{R_{\chi}}=\{z\}$, then $\mathbb{w}_{\alpha_{r}}[z]=z \in \mathscr{X}_{Q, n}$. Write $z=\sum_{i=1}^{r} z_{i} e_{i} \in \mathscr{X}_{Q, n}$ with $0 \leq z_{i} \leq n-1$. The equality $\mathbb{w}_{\alpha_{r}}[z]=z$ is equivalent to that $z_{r}=(n+1) / 2$. It follows from [GSS, Proposition 4.12] that

$$
\tau\left(w_{\alpha_{r}}, \chi, \mathbf{s}_{z}, \mathbf{s}_{z}\right)=\chi_{\alpha_{r}}^{2} \cdot \gamma\left(w_{\alpha_{r}}, \chi\right)^{-1}=1 \cdot \gamma\left(w_{\alpha_{r}}, \chi\right)^{-1} .
$$


That is, (36) holds for such $\mathcal{O}_{z}^{R_{\chi}}$. In fact, we also see that the character $\theta_{\sigma} \mathscr{X}$ of the representation $\sigma^{\mathscr{X}}: R_{\chi} \rightarrow \operatorname{Perm}\left(\mathscr{X}_{Q, n}\right)$ is given by

$$
\theta_{\sigma \mathscr{X}}(1)=n^{r}, \quad \theta_{\sigma} \mathscr{X}\left(\mathbb{w}_{\alpha_{r}}\right)=n^{r-1} .
$$

Second, assume $\mathcal{O}_{z}^{R_{\chi}}=\left\{z, \mathbb{w}_{\alpha_{r}}[z]\right\}$, then $n \nmid\left\langle z_{\rho}, \alpha_{r}\right\rangle$. It follows from the proof of [Gao17, Lemma 3.9] that

We obtain

$$
k_{z, \alpha_{r}}+k_{\mathrm{w} \alpha_{r}[z], \alpha_{r}}=1 \text {. }
$$

$$
\begin{aligned}
& \tau\left(w_{\alpha_{r}}, \chi, \mathbf{s}_{z}, \mathbf{s}_{z}\right)+\tau\left(w_{\alpha_{r}}, \chi, \mathbf{s}_{\mathbb{W}_{\alpha_{r}}[z]}, \mathbf{s}_{\mathrm{w}_{\alpha_{r}}[z]}\right) \\
= & \frac{1-q^{-1}}{1-\chi_{\alpha_{r}}}\left(\chi_{\alpha_{r}}\right)^{k_{z, \alpha_{r}}}+\frac{1-q^{-1}}{1-\chi_{\alpha_{r}}}\left(\chi_{\alpha_{r}}\right)^{k_{\mathrm{w} \alpha_{r}[z], \alpha_{r}}} \\
= & \frac{1-q^{-1}}{2}(-1)^{k_{z, \alpha_{r}}}+\frac{1-q^{-1}}{2}(-1)^{1-k_{z, \alpha_{r}}} \\
= & 0 .
\end{aligned}
$$

On the other hand, $\left|\left(\mathcal{O}_{z}^{R_{\chi}}\right)^{\mathrm{w} \alpha_{r}}\right|=0$; this shows that (36) holds in this case.

Therefore Conjecture 5.7 holds for $\overline{\mathrm{Sp}}_{2 r}^{(n)}$. The desired dimension formula for the Whittaker spaces of $\pi_{\chi}^{u n}$ and $\pi_{\varepsilon}$ follows from Theorem 5.6 and the character $\theta_{\sigma} \mathscr{X}$ computed above. This completes the proof.

Remark 7.2. The equality in (36) might fail for covers of a general simply-connected group. More precisely, for a general $\bar{G}$, we have the decomposition of a $W$-orbit $\mathcal{O}_{y}$ into $R_{\chi}$-orbits

$$
\mathcal{O}_{y}=\bigsqcup_{i \in I} \mathcal{O}_{z_{i}}^{R_{\chi}}
$$

Conjecture 5.7 predicts that for every $\mathbb{w} \in R_{\chi}$, one has

$$
\sum_{i \in I} \sum_{z^{\prime} \in \mathcal{O}_{z_{i}}^{R_{\chi}}} \tau\left(w, \chi, \mathbf{s}_{z^{\prime}}, \mathbf{s}_{z^{\prime}}\right)=\sum_{i \in I}\left|\left(\mathcal{O}_{z_{i}}^{R_{\chi}}\right)^{\mathrm{w}}\right| \cdot \gamma(w, \chi)^{-1} .
$$

However, the inner summands indexed by $I$ on the two sides may not be equal. A counterexample arises from considering $\overline{\mathrm{SL}}_{4}^{(3)}$ with $y=\sum_{\alpha \in \Delta} \alpha^{\vee}$, in which case $\left|\mathcal{O}_{y}\right|=6$.

This subtlety is of the main difficulty with verifying Conjecture 5.7 by a direct computation. Indeed, it follows from Table 1 and Table 2 (or more precisely [Key82b, §3]) that the nontrivial unramified group $R_{\chi}$ for covers of simply-connected groups of type $B_{r}, D_{r}, E_{6}$ and $E_{7}$ is small; thus the orbits $\mathcal{O}_{z_{i}}^{R_{\chi}}$ are all small. However, as just noted, one needs to consider the whole $W$-orbit $\mathcal{O}_{y}$, whose size could be large depending on $W$ and $n$. This hinders one from a direct computation in the general situation.

Theorem 7.1 could also be obtained from the consideration in $\S 6.2$, especially Conjecture 6.4. We illustrate this by considering the case of $\overline{\mathrm{SL}}_{2}^{(n)}$. Write $n=2 d+1$ and $\Delta=\{\alpha\}$. The twisted Weyl action on $\mathscr{X}_{Q, n}=\mathbf{Z} / n \mathbf{Z}$ is given by

$$
\mathrm{w}_{\alpha}\left[k \alpha^{\vee}\right]=(1-k) \alpha^{\vee} \in \mathscr{X}_{Q, n} .
$$

In total there are $(d+1)$-many $W$-orbits. Every orbit except that of $-d \alpha^{\vee}$ is free. We choose a set of representatives of $\mathscr{X}_{Q, n}$ as

$$
\mathfrak{R}=\left\{i \cdot \alpha^{\vee}:-d \leq i \leq d\right\} .
$$

The $W$-orbits in $\mathscr{X}_{Q, n}$ are

$$
\mathcal{O}_{\mathscr{X}}=\left\{\mathcal{O}_{i \alpha^{\vee}}: 1 \leq i \leq d\right\} \cup\left\{\mathcal{O}_{-d \alpha^{\vee}}\right\}
$$


with

$$
\mathfrak{R}_{i \alpha^{\vee}}=\left\{i \alpha^{\vee},(1-i) \alpha^{\vee}\right\} \text { for } 1 \leq i \leq d \text { and } \mathfrak{R}_{-d \alpha^{\vee}}=\left\{-d \alpha^{\vee}\right\} .
$$

Proposition 7.3. Conjecture 6.4 holds for $\mathfrak{R}_{i \alpha^{\vee}}$ and moreover

$$
\operatorname{rank}\left(\nu_{\mathfrak{R}_{i \alpha} \vee}^{\chi}\right)=1
$$

for every $1 \leq i \leq d$ and $i=-d$.

Proof. Since $-d \alpha^{\vee} \in Y$ is an exceptional point, in view of Theorem 6.5, it suffices to deal with the case $1 \leq i \leq d$. We denote $z:=i \alpha^{\vee}$. Let $\lambda_{\mathbf{c}} \in \mathrm{Wh}_{\psi}(I(\chi))_{\mathcal{O}_{z}}$, viewed as an element in $\mathrm{Wh}_{\psi}\left(\pi_{\chi}^{u n}\right)_{\mathcal{O}_{z}}$ by restriction. Then

$$
\operatorname{supp}(\mathbf{c}) \subset \mathbf{s}_{z} \cdot \bar{A} \cup \mathbf{s}_{\mathrm{w}[z]} \cdot \bar{A} .
$$

We have

$$
\mathcal{W}_{\mathbf{c}}^{*}=\mathbf{c}\left(\mathbf{s}_{z}\right) \cdot \mathcal{W}_{\mathbf{s}_{z}}^{*}+\mathbf{c}\left(\mathbf{s}_{\mathrm{w}[z]}\right) \cdot \mathcal{W}_{\mathbf{s}_{\mathrm{ww}[z]}^{*}}^{*}
$$

Recall the projection map $h^{u n}: \mathrm{Wh}_{\psi}(I(\chi))_{\mathcal{O}_{z}} \rightarrow \mathrm{Wh}_{\psi}\left(\pi_{\chi}^{u n}\right)$ from $\S 6.2$. Assume $\mathbf{c}$ is such that

$$
\nu_{\mathfrak{R}_{z}}^{\chi}\left(\lambda_{\mathbf{c}}\right)=\left(\mathcal{W}_{\mathbf{c}}^{*}\left(\mathbf{s}_{z}\right), \mathcal{W}_{\mathbf{c}}^{*}\left(\mathbf{s}_{\mathbf{w}[z]}\right)\right)=(0,0) .
$$

We want to show that $h^{u n}\left(\lambda_{\mathbf{c}}\right)=0$. For each $z=i \alpha^{\vee}$, we denote

$$
\mathcal{M}_{\mathfrak{R}_{z}}:=\left(\begin{array}{cc}
\mathcal{W}_{\mathbf{s}_{z}}^{*}\left(\mathbf{s}_{z}\right) & \mathcal{W}_{\mathbf{s}_{z}}^{*}\left(\mathbf{s}_{\mathrm{ww}[z]}\right) \\
\mathcal{W}_{\mathbf{s}_{\mathrm{w}[z]}}^{*}\left(\mathbf{s}_{z}\right) & \mathcal{W}_{\mathbf{s}_{\mathrm{w}[z]}^{*}}^{*}\left(\mathbf{s}_{\mathrm{w}[z]}\right)
\end{array}\right)
$$

It is easy to see that

$$
\nu_{\mathfrak{R}_{z}}^{\chi}\left(\lambda_{\mathbf{c}}\right)=\left(\mathbf{c}\left(\mathbf{s}_{z}\right), \mathbf{c}\left(\mathbf{s}_{\mathbb{w}[z]}\right)\right) \mathcal{M}_{\mathfrak{R}_{z}} .
$$

Now by (32), one has

$$
\mathcal{W}_{\mathbf{s}_{z}}^{*}\left(\mathbf{s}_{y}\right) \cdot \delta_{B}^{1 / 2}\left(\mathbf{s}_{y}\right)=c_{\mathrm{gk}}\left(w_{\alpha}, \chi\right) \cdot \tau\left(\mathrm{id}, \chi, \mathbf{s}_{z}, \mathbf{s}_{y}\right)+\tau\left(w_{\alpha},{ }^{w_{\alpha}} \chi, \mathbf{s}_{z}, \mathbf{s}_{y}\right) .
$$

Since we are in the case where $\underline{\chi}_{\alpha}^{2}=\mathbb{1}$ but $\underline{\chi}_{\alpha} \neq \mathbb{1}$, we have

$$
\underline{\chi}_{\alpha}(\varpi)=\chi\left(\bar{h}_{\alpha}\left(\varpi^{n}\right)\right)=-1 \text {. }
$$

We also note that ${ }^{w_{\alpha}} \chi=\chi$. Thus a straightforward computation gives that

$$
\mathcal{M}_{\mathfrak{\Re}_{i \alpha} \vee}=\left(\begin{array}{cc}
\mathcal{W}_{\mathbf{s}_{i \alpha \vee}}^{*}\left(\mathbf{s}_{i \alpha \vee}\right) & \mathcal{W}_{\mathbf{s}_{i \alpha} \vee}^{*}\left(\mathbf{s}_{(1-i) \alpha^{\vee}}\right) \\
\mathcal{W}_{\mathbf{s}_{(1-i) \alpha}}^{*}\left(\mathbf{s}_{i \alpha^{\vee}}\right) & \mathcal{W}_{\mathbf{s}_{(1-i) \alpha}}^{*}\left(\mathbf{s}_{(1-i) \alpha^{\vee}}\right)
\end{array}\right)=\left(\begin{array}{cc}
q^{i-1} & q^{-i-1} \mathbf{g}_{\psi^{-1}}(1-2 i) \\
q^{i} \mathbf{g}_{\psi^{-1}}(2 i-1) & q^{-i-1} .
\end{array}\right) .
$$

Combining (38) and (39), we get

$$
q^{-1} \cdot \mathbf{c}\left(\mathbf{s}_{i \alpha^{\vee}}\right)+\mathbf{g}_{\psi^{-1}}(2 i-1) \cdot \mathbf{c}\left(\mathbf{s}_{(1-i) \alpha^{\vee}}\right)=0 .
$$

Consider the map

$$
P_{\mathbb{1}}^{*}: \mathrm{Wh}_{\psi}(I(\chi)) \longleftrightarrow \mathrm{Wh}_{\psi}(I(\chi))
$$

induced from the projection $P_{\mathbb{1}}: I(\chi) \rightarrow I(\chi)$. To show $h^{\text {un }}\left(\lambda_{\mathbf{c}}\right)=0$ is equivalent to proving $P_{\mathbb{1}}^{*}\left(\lambda_{\mathbf{c}}\right)=0$. Now

$$
\begin{aligned}
& P_{\mathbb{1}}^{*}\left(\lambda_{\mathbf{c}}\right) \\
= & \frac{1}{2} \cdot\left(\lambda_{\mathbf{c}}+\mathscr{A}(w, \chi)^{*}\left(\lambda_{\mathbf{c}}\right)\right) \\
= & \frac{1}{2}\left(\mathbf{c}\left(\mathbf{s}_{z}\right)+\mathbf{c}\left(\mathbf{s}_{z}\right) \gamma(w, \chi) \tau(w, \chi, z, z)+\mathbf{c}\left(\mathbf{s}_{\mathbb{w}[z]}\right) \gamma(w, \chi) \tau(w, \chi, \mathbb{w}[z], z)\right) \cdot \lambda_{\mathbf{s}_{z}} \\
& +\frac{1}{2}\left(\mathbf{c}\left(\mathbf{s}_{\mathbb{w}[z]}\right)+\mathbf{c}\left(\mathbf{s}_{z}\right) \gamma(w, \chi) \tau(w, \chi, z, \mathbb{w}[z])+\mathbf{c}\left(\mathbf{s}_{\mathbb{w}[z]}\right) \gamma(w, \chi) \tau(w, \chi, \mathbb{w}[z], \mathbb{w}[z])\right) \cdot \lambda_{\mathbf{s}_{\mathbb{w}[z]}} .
\end{aligned}
$$


A simplification gives that the above coefficient in front of $\lambda_{\mathbf{s}_{z}}$ is

$$
\frac{1}{1+q^{-1}} \cdot\left(\mathbf{c}\left(\mathbf{s}_{i \alpha^{\vee}}\right) q^{-1}+\mathbf{c}\left(\mathbf{s}_{(1-i) \alpha^{\vee}}\right) \mathbf{g}_{\psi^{-1}}(2 i-1)\right),
$$

which is equal to 0 by (40). Similarly, it can be checked easily that the coefficient in front of $\lambda_{\mathbf{s}_{\mathrm{ww}[z]}}$ is also 0. This shows that Conjecture 6.4 holds.

It is clear that for every $1 \leq i \leq d$, we have

$$
\operatorname{rank}\left(\nu_{\mathfrak{R}_{i \alpha} \vee}^{\chi}\right)=\operatorname{rank}\left(\mathcal{M}_{\mathfrak{R}_{i \alpha} \vee}\right)=1 .
$$

The proof is now completed.

It follows from Proposition 7.3 that

$$
\operatorname{dim} \mathrm{Wh}_{\psi}\left(\pi_{\chi}^{u n}\right)=\left\langle\mathbb{1}, \sigma^{\mathscr{X}}\right\rangle_{R_{\chi}}=\left|\mathcal{O}_{\mathscr{X}}\right|=d+1=\frac{n+1}{2} .
$$

Consequently,

$$
\operatorname{dim} \mathrm{Wh}_{\psi}\left(\pi_{\varepsilon}\right)=\left\langle\varepsilon, \sigma^{\mathscr{X}}\right\rangle_{R_{\chi}}=d=\frac{n-1}{2},
$$

the number of free $R_{\chi}$-orbits in $\mathscr{X}_{Q, n}$.

Remark 7.4. Let $n$ be odd and $\chi$ be the above non-trivial quadratic genuine character of $Z(\bar{T}) \subset \overline{\mathrm{Sp}}_{2 r}$. The Whittaker dimension for the constituents in $\pi_{\chi}^{u n} \oplus \pi_{\chi}^{\prime}=\operatorname{Ind} \frac{\overline{\mathrm{Sp}}_{2} r}{B}(i(\chi))$ can be deduced from that of $\overline{\mathrm{SL}}_{2}^{(n)}$ as follows. Here we write $\pi_{\chi}^{\prime}$ for $\pi_{\varepsilon}$. Each of the rank-one lattice $\left(\mathbf{Z} e_{j}\right) \subset Y$ gives rise to an $n$-fold covering $\overline{\mathrm{GL}}_{1, e_{j}}$ of the torus $\mathrm{GL}_{1} \simeq F^{\times}$, by restriction from $\bar{T}$. One has an isomorphism (see [GW19, §5.1.3])

$$
\prod_{1 \leq j \leq r} \overline{\mathrm{GL}}_{1, e_{j}} / H \simeq \bar{T}
$$

where $H=\left\{\left(\zeta_{j}\right) \in\left(\mu_{n}\right)^{r}: \prod_{j} \zeta_{j}=1\right\}$; that is, block-commutativity holds for coverings of Levi-subgroups of $\overline{\mathrm{Sp}}_{2 r}$. Thus, we can write

$$
i(\chi)=\prod_{j=1}^{r} i\left(\chi_{j}\right),
$$

where $i\left(\chi_{j}\right) \in \operatorname{Irr}\left(\overline{\mathrm{GL}}_{1, e_{j}}\right)$ is of dimension $n$. Note also $\bar{T}_{0}:=\overline{\mathrm{GL}}_{1, e_{r}}$ is just the covering torus of $\overline{\mathrm{SL}}_{2}$ associated to $\alpha_{r}$. Let $\bar{M}=\prod_{1 \leq j \leq r-1} \overline{\mathrm{GL}}_{1, e_{j}} \times \overline{\mathrm{SL}}_{2}$ be the Levi subgroup of the parabolic subgroup $\bar{P} \subset \overline{\mathrm{Sp}}_{2 r}^{(n)}$ associated to $\alpha_{r}$. The character $\chi_{r}$ is a non-trivial quadratic character of $Z\left(\bar{T}_{0}\right)$ and thus we have

$$
\operatorname{Ind}_{\bar{T}_{0}}^{\overline{\mathrm{SL}}_{2}}\left(i\left(\chi_{r}\right)\right)=\pi_{\chi_{r}}^{u n} \oplus \pi_{\chi_{r}}^{\prime}
$$

By induction in stages, one has

$$
\pi_{\chi}^{u n}=\operatorname{Ind} \overline{\mathrm{Sp}}_{\bar{P}}{ }^{2 r}\left(\bigotimes_{1 \leq j \leq r-1} i\left(\chi_{j}\right)\right) \otimes \pi_{\chi_{r}}^{u n}
$$

and similarly,

$$
\pi_{\chi}^{\prime}=\operatorname{Ind} \overline{\mathrm{Sp}}_{2 r}\left(\bigotimes_{1 \leq j \leq r-1} i\left(\chi_{j}\right)\right) \otimes \pi_{\chi_{r}}^{\prime} .
$$

Now it follows from the equalities

$$
\operatorname{dim} i\left(\chi_{j}\right)=n, \quad \operatorname{dim} \mathrm{Wh}_{\psi}\left(\pi_{\chi_{r}}^{u n}\right)=\frac{n+1}{2}, \quad \operatorname{dim} \mathrm{Wh}_{\psi}\left(\pi_{\chi_{r}}^{\prime}\right)=\frac{n-1}{2}
$$


and Rodier's heredity that

$$
\operatorname{dim} \mathrm{Wh}_{\psi}\left(\pi_{\chi}^{u n}\right)=n^{r-1} \cdot \frac{n+1}{2}, \quad \operatorname{dim} \mathrm{Wh}_{\psi}\left(\pi_{\chi}^{\prime}\right)=n^{r-1} \cdot \frac{n-1}{2},
$$

which agrees with Theorem 7.1

7.2. Double cover of $\mathrm{SL}_{3}$. Before we proceed, we recall some observations from $§ 5.4$. By the tables in $§ 4.4$ and Corollary 4.6 , the group $R_{\chi}$ is always cyclic for all semisimple type except the $D_{r}$ case when $r$ is even. Recall that

$$
\mathscr{A}(w, \chi)^{*}=\gamma(w, \chi) \cdot A(w, \chi)^{*} .
$$

Assume $R_{\chi}$ is cyclic and let $\mathbb{w}$ be a generator; then $\sigma(\mathbb{w}), \sigma \in \operatorname{Irr}\left(R_{\chi}\right)$ are distinct and $\operatorname{dim} \mathrm{Wh}_{\psi}\left(\pi_{\sigma}\right), \sigma \in \operatorname{Irr}\left(R_{\chi}\right)$ are just the multiplicities of the distinct eigenvalues $\sigma(\mathbb{w})$. $\gamma(w, \chi)^{-1}$ of the polynomial

$$
\operatorname{det}\left(X \cdot \operatorname{id}-A(w, \chi)^{*}\right)=\operatorname{det}\left(X \cdot I_{\left|\mathscr{X}_{Q, n}\right|}-\mathcal{S}_{\mathfrak{R}}(w, i(\chi))\right) .
$$

This will be the observation we apply to the double cover $\overline{\mathrm{SL}}_{3}^{(2)}$ in this subsection.

Let $\alpha_{1}^{\vee}, \alpha_{2}^{\vee}$ be the two simple co-roots of $\mathrm{SL}_{3}$ :

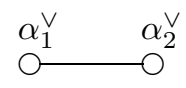

For convenience, we write $\mathbb{w}_{i}=\mathbb{w}_{\alpha_{i}}$ for $i=1,2$. Let $\alpha_{3}=\alpha_{1}^{\vee}+\alpha_{2}^{\vee} \in \Phi^{+}$. Let $Q: Y \rightarrow \mathbf{Z}$ be the unique Weyl-invariant quadratic form such that $Q\left(\alpha_{1}^{\vee}\right)=1$. Taking $n=2$, we get

$$
Y_{Q, 2}=Y_{Q, 2}^{s c}=2 Y
$$

and thus

The ordered set

$$
\mathscr{X}_{Q, 2} \simeq(\mathbf{Z} / 2 \mathbf{Z}) \oplus(\mathbf{Z} / 2 \mathbf{Z})
$$

$$
\mathfrak{R}=\left\{0, \alpha_{1}^{\vee}, \alpha_{2}^{\vee}, \alpha_{3}^{\vee}\right\} \subset Y
$$

is a set of representatives of $\mathscr{X}_{Q, 2}$. There are two $W$-orbits

$$
\mathcal{O}_{0}=\left\{0, \alpha_{1}^{\vee}, \alpha_{2}^{\vee}\right\}, \quad \mathcal{O}_{\alpha_{3}^{\vee}}=\left\{\alpha_{3}^{\vee}\right\} .
$$

Let $\chi$ be a unitary unramified genuine character of $Z(\bar{T})$. Since the dual group of $\overline{\mathrm{SL}}_{3}^{(2)}$ is $\mathrm{PGL}_{3}$, we see that $R_{\chi}^{s c}=R_{\chi}$ by Proposition 4.4 ; moreover, $R_{\chi}$ is either trivial or $\mathbf{Z} / 3 \mathbf{Z}$.

Assume $R_{\chi}=\mathbf{Z} / 3 \mathbf{Z}$, we see that $\Phi_{\chi}=\emptyset$ and $R_{\chi}=W_{\chi}=\left\langle\mathbb{w}_{1} \mathbb{w}_{2}\right\rangle=\left\langle\mathbb{w}_{2} \mathbb{w}_{1}\right\rangle \subset W$, i.e.

$$
{ }^{\mathrm{w}_{2} \mathrm{w}_{1}} \chi=\chi \text {. }
$$

This implies that

$$
\chi_{\alpha_{1}}=\zeta=\chi_{\alpha_{2}}, \text { and } \chi_{\alpha_{3}}=\zeta^{2},
$$

where $\zeta \in \mathbf{C}^{\times}$is a primitive third-root of unity. For such $\chi$, one has the decomposition

$$
I(\chi)=\pi_{\chi}^{u n} \oplus \pi_{1} \oplus \pi_{2}
$$

of $I(\chi)$ into non-isomorphic irreducible components. We have $\operatorname{dim} \mathrm{Wh}_{\psi}(I(\chi))=4$, and the permutation representation

$$
\sigma^{\mathscr{X}}: R_{\chi} \longrightarrow \operatorname{Perm}\left(\mathscr{X}_{Q, 2}\right)
$$

is such that in $\mathscr{X}_{Q, 2}$ :

$$
\mathbb{w}_{2} \mathbb{w}_{1}\left[\alpha_{3}^{\vee}\right]=\alpha_{3}^{\vee}, \quad \mathbb{w}_{2} \mathbb{w}_{1}[0]=\alpha_{1}^{\vee}, \quad \mathbb{w}_{2} \mathbb{w}_{1}\left[\alpha_{1}^{\vee}\right]=\alpha_{2}^{\vee}, \quad \mathbb{w}_{2} \mathbb{w}_{1}\left[\alpha_{2}^{\vee}\right]=\alpha_{0}^{\vee} .
$$

We have $\operatorname{Irr}\left(R_{\chi}\right)=\left\{\mathbb{1}, \sigma, \sigma^{2}\right\}$ where $\sigma$ is the generator given by

$$
\sigma\left(\mathbb{w}_{2} \mathbb{w}_{1}\right)=\zeta .
$$


It then follows easily that

$$
\sigma^{\mathscr{X}}=(2 \cdot \mathbb{1}) \oplus \sigma \oplus \sigma^{2} .
$$

Thus, we could label constituents of $I(\chi)$ as

$$
\pi_{\chi}^{u n}=\pi_{\mathbb{1}}=\pi_{\sigma^{0}}, \quad \pi_{1}=\pi_{\sigma} \text { and } \pi_{2}=\pi_{\sigma^{2}} .
$$

Since $R_{\chi}$ is cyclic and $\mathbb{w}_{2} \mathbb{w}_{1}$ is a generator of $\operatorname{Irr}\left(R_{\chi}\right)$, to determine $\operatorname{dim} \operatorname{Wh}_{\psi}\left(\pi_{\sigma^{i}}\right)$, it suffices to compute the characteristic polynomial of $A\left(w_{2} w_{1}, \chi\right)^{*}$ which takes the form

$$
\operatorname{det}\left(X \cdot \mathrm{id}-A\left(w_{2} w_{1}, \chi\right)^{*}\right)=\prod_{0 \leq i \leq 2}\left(X-\zeta^{i} \cdot \gamma\left(w_{2} w_{1}, \chi\right)^{-1}\right)^{\operatorname{dim} \mathrm{Wh}_{\psi}\left(\pi_{\sigma^{i}}\right)},
$$

where

$$
\gamma\left(w_{2} w_{1}, \chi\right)^{-1}=\frac{\left(1-q^{-1} \chi_{\alpha_{1}}^{-1}\right)\left(1-q^{-1} \chi_{\alpha_{3}}\right)^{-1}}{\left(1-\chi_{\alpha_{1}}\right)\left(1-\chi_{\alpha_{3}}\right)}=\frac{1+q^{-1}+q^{-2}}{3} .
$$

Let $\mathcal{S}_{\mathfrak{R}}\left(w_{2} w_{1}, i(\chi)\right)$ be the scattering matrix associated to the ordered set $\mathfrak{R}$ above. For simplicity of computation, we assume $\mu_{4} \subset F^{\times}$, and hence $\varepsilon=1$. By using (25), (26) and Theorem 5.2, we obtain in this case an explicit form (again, using the short-hand notation $\left.\chi_{\alpha_{1}}, \chi_{\alpha_{2}}, \chi_{\alpha_{3}}\right)$ :

$$
\begin{aligned}
\mathcal{S}_{\mathfrak{R}}\left(w_{2} w_{1}, i(\chi)\right) & \left(\begin{array}{cccc}
\frac{\left(1-q^{-1}\right)^{2}}{\left(1-\chi_{\alpha_{1}}\right)\left(1-\chi_{\alpha_{3}}\right)} & \mathbf{g}_{\psi^{-1}}(-1) \gamma\left(\underline{\chi}_{\alpha_{3}}\right)^{-1} & \mathbf{g}_{\psi^{-1}}(-1) \frac{1-q^{-1}}{1-\chi_{\alpha_{1}}} & 0 \\
\mathbf{g}_{\psi^{-1}}(1) \frac{1-q^{-1}}{1-\chi_{\alpha_{3}}} & \chi_{\alpha_{1}} \frac{1-q^{-1}}{1-\chi_{\alpha_{1}}} \gamma\left(\underline{\chi}_{\alpha_{3}}\right)^{-1} & q^{-1} & 0 \\
\mathbf{g}_{\psi^{-1}}(1) \gamma\left(\underline{\chi}_{\alpha_{1}}\right)^{-1} & 0 & \chi_{\alpha_{3}} \frac{1-q^{-1}}{1-\chi_{\alpha_{3}}} \gamma\left(\underline{\chi}_{\alpha_{1}}\right)^{-1} & 0 \\
0 & 0 & 0 & \chi_{\alpha_{1}} \chi_{\alpha_{3}} \gamma\left(\underline{\chi}_{\alpha_{1}}\right)^{-1} \gamma\left(\underline{\chi}_{\alpha_{3}}\right)^{-1}
\end{array}\right)
\end{aligned}
$$

A straightforward computation gives that

$$
\begin{aligned}
& \operatorname{det}\left(X \cdot I_{4}-\mathcal{S}_{\mathfrak{R}}\left(w_{2} w_{1}, i(\chi)\right)\right) \\
= & \left(X-\frac{1+q^{-1}+q^{-2}}{3}\right) \cdot\left(X^{3}-\left(\frac{1+q^{-1}+q^{-2}}{3}\right)^{3}\right),
\end{aligned}
$$

and thus

$$
\operatorname{det}\left(X \cdot I_{4}-\mathscr{A}\left(w_{2} w_{1}, \chi\right)^{*}\right)=(X-1)^{2} \cdot(X-\zeta) \cdot\left(X-\zeta^{2}\right) \text {. }
$$

Therefore,

Clearly,

$$
\operatorname{dim} \mathrm{Wh}_{\psi}\left(\pi_{\mathbb{1}}\right)=2 \text { and } \operatorname{dim} \mathrm{Wh}_{\psi}\left(\pi_{\sigma^{i}}\right)=1 \text { for } i=1,2 .
$$

$$
\sigma^{\mathrm{Wh}}=(2 \cdot \mathbb{1}) \oplus \sigma \oplus \sigma^{2}
$$

Proposition 7.5. For $\overline{\mathrm{SL}}_{3}^{(2)}$ we have

$$
\sigma^{\mathrm{Wh}}=\sigma^{\mathscr{X}}=(2 \cdot \mathbb{1}) \oplus \sigma \oplus \sigma^{2} .
$$

Moreover, Conjecture 5.7 holds.

Proof. The equalities are clear. It suffices to prove that Conjecture 5.7 holds for the two orbits $\mathcal{O}_{0}$ and $\mathcal{O}_{\alpha_{3}^{v}}$, which is a priori stronger than the equalities. This either follows from a direct computation, or we argue alternatively by using the fact that $\mathcal{O}_{\mathscr{X}}=\left\{\mathcal{O}_{0}, \mathcal{O}_{\alpha_{3}^{\vee}}\right\}$ with $\alpha_{3}^{\vee} \in \mathscr{X}_{Q, 2}^{\text {exc }}$. Indeed, by Theorem 6.1, $\sigma_{\mathcal{O}_{3}^{\vee}}^{\mathrm{Wh}}=\sigma_{\mathcal{O}_{\alpha_{3}^{\vee}}}^{\mathrm{Wh}}=\mathbb{1}$. However, since

it enforces that

$$
\sigma^{\mathrm{Wh}}=\sigma_{\mathcal{O}_{\alpha_{3}}}^{\mathrm{Wh}} \oplus \sigma_{\mathcal{O}_{0}}^{\mathrm{Wh}}=\sigma_{\mathcal{O}_{\alpha_{3}}}^{\mathscr{X}} \oplus \sigma_{\mathcal{O}_{0}}^{\mathscr{X}}=\sigma^{\mathscr{X}},
$$

The proof is completed.

$$
\sigma_{\mathcal{O}_{0}}^{\mathrm{Wh}}=\sigma_{\mathcal{O}_{0}}^{\mathrm{Wh}}=\mathbb{1} \oplus \sigma \oplus \sigma^{2}
$$




\section{TWO REMARKS}

In this section, we consider two examples to justify the necessary constraints imposed on $G$ and $\bar{G}$ in Conjecture 5.7. First, we consider $\overline{\mathrm{SO}}_{3}^{(n)}$ and show that a naive analogous formula does not hold for general covers of semisimple groups which are not simply connected. Second, we consider the double cover of the simply-connected $\mathrm{Spin}_{6} \simeq \mathrm{SL}_{4}$, whose dual group is $\mathrm{SL}_{4} / \mu_{2}$, and show that analogous Conjecture 5.7 does not hold. This shows that it is necessary to require the cover $\bar{G}$ to be saturated.

8.1. Covers of $\mathrm{SO}_{3}$. Let $Y=\mathbf{Z} \cdot e$ be the cocharacter lattice of $\mathrm{SO}_{3}$ with $\alpha^{\vee}=2 e$ generating the co-root lattice $Y^{s c}$. Let $Q: Y \rightarrow \mathbf{Z}$ be the Weyl-invariant quadratic form such that $Q(e)=1$. Thus, $Q\left(\alpha^{\vee}\right)=4$. We get

$$
\alpha_{Q, n}^{\vee}=\frac{n}{\operatorname{gcd}(4, n)} \cdot \alpha^{\vee}
$$

On the other hand,

$$
Y_{Q, n}=\mathbf{Z} \cdot \frac{n}{\operatorname{gcd}(2, n)} e .
$$

The equality ${ }^{w_{\alpha}} \chi=\chi$ is equivalent to that

$$
\chi\left(\bar{h}_{\alpha}\left(a^{n / \operatorname{gcd}(2, n)}\right)\right)=1
$$

for all $a \in F^{\times}$.

Lemma 8.1. Let $\chi$ be a unitary unramified genuine character of $Z(\bar{T})$. Then $R_{\chi}=W$ if and only if $4 \mid n$ and $\underline{\chi}_{\alpha}$ is a non-trivial quadratic character.

Proof. Clearly, $R_{\chi}=W$ if and only if $\Phi_{\chi}=\emptyset$ and $W_{\chi}=W$. We discuss case by case. First, if $4 \nmid n$, then $\operatorname{gcd}(4, n)=\operatorname{gcd}(2, n)$, and in this case, $R_{\chi}=\{1\}$. Second, if $n=4 m$, then $\alpha_{Q, n}^{\vee}=m \alpha^{\vee}$ and $n / \operatorname{gcd}(2, n)=2 m$. In this case, if $\underline{\chi}_{\alpha}$ is a non-trivial quadratic character, then we have $R_{\chi}=W$.

Remark 8.2. For $G$ of adjoint type, if $\chi$ is a unitary unramified character of $T$, then $I(\chi)$ is always irreducible (see Corollary 4.10). The above result shows that this may fail for covers of groups of adjoint type.

Now we assume that $n=4 m$ and $\underline{\chi}_{\alpha}$ is a non-trivial quadratic character, i.e.

$$
\chi_{\alpha}=\chi\left(\bar{h}_{\alpha}\left(\varpi^{n_{\alpha}}\right)\right)=-1 .
$$

In this case, $R_{\chi}=W$ and

We have

$$
I(\chi)=\pi_{\chi}^{u n} \oplus \pi
$$

$$
Y_{Q, n}=Y_{Q, n}^{s c}=\mathbf{Z} \cdot \alpha_{Q, n}^{\vee}=\mathbf{Z} \cdot\left(m \alpha^{\vee}\right)=\mathbf{Z} \cdot(2 m e) .
$$

Therefore, $\bar{G}^{\vee} \simeq \mathrm{SO}_{3}$ is of adjoint type. It is clear that

$$
\mathscr{X}_{Q, n} \simeq \mathbf{Z} /(2 m) \mathbf{Z}
$$

with the twisted Weyl action given by

$$
\mathbb{w}_{\alpha}[i e]=(-i) e+2 e=(2-i) e .
$$

One has $\left|\mathcal{O}_{\mathscr{X}}\right|=m+1$, i.e., there are $m+1$ many $W$-orbits in $\mathscr{X}_{Q, n}$. Let $\mathfrak{R} \subset Y$ be the following set of representatives of $\mathscr{X}_{Q, n}$ :

$$
\mathfrak{R}=\{i e:-m+1 \leq i \leq m\} \text {. }
$$


The two trivial $W$-orbits are

$$
\mathcal{O}_{e}=\{e\}, \quad \mathcal{O}_{(-m+1) e}=\{(-m+1) e\} ;
$$

while for all other $i e \in \mathfrak{R}$ with $2 \leq i \leq m$, the orbit

$$
\mathcal{O}_{i e}=\{i e,(2-i) e\} \subset \mathscr{X}_{Q, n}
$$

is $W$-free. We thus have $\mathfrak{R}_{e}, \mathfrak{R}_{(-m+1) e}$ and $\mathfrak{R}_{i e} \subset \mathfrak{R}$ to represent the above three families of orbits.

Proposition 8.3. Assume $n=4 m$ and $\underline{\chi}_{\alpha}$ is a non-trivial quadratic character. Then

$$
\sigma^{\mathrm{Wh}}=m \cdot \mathbb{1}_{W} \oplus m \cdot \varepsilon_{W}, \text { and } \sigma^{\mathscr{X}}=(m+1) \cdot \mathbb{1}_{W} \oplus(m-1) \cdot \varepsilon_{W} .
$$

Hence,

$$
\operatorname{dim} \mathrm{Wh}_{\psi}\left(\pi_{\chi}^{u n}\right)=m=\operatorname{dim} \mathrm{Wh}_{\psi}(\pi) .
$$

Proof. Choosing $\mathfrak{R}$ as above, the scattering matrix $\mathcal{S}_{\mathfrak{R}}\left(w_{\alpha}, i(\chi)\right)$ is the block-diagonal matrix with blocks $\mathcal{S}_{\mathfrak{R}}\left(w_{\alpha}, i(\chi)\right)_{\mathcal{O}_{i e}}$ for $i=-m+1$ and $1 \leq i \leq m$. Here,

$$
\mathcal{S}_{\mathfrak{R}}\left(w_{\alpha}, i(\chi)\right)_{\mathcal{O}_{(-m+1) e}}=\gamma\left(w_{\alpha}, \chi\right)^{-1}=\frac{1-q^{-1} \chi_{\alpha}}{1-\chi_{\alpha}}=\frac{1+q^{-1}}{2} .
$$

and

$$
\mathcal{S}_{\mathfrak{R}}\left(w_{\alpha}, i(\chi)\right)_{\mathcal{O}_{e}}=\chi_{\alpha} \cdot \gamma\left(w_{\alpha}, \chi\right)^{-1}=-\frac{1+q^{-1}}{2}
$$

also, for $2 \leq i \leq m$,

$$
\mathcal{S}_{\mathfrak{R}}\left(w_{\alpha}, i(\chi)\right)_{\mathcal{O}_{i e}}=\left(\begin{array}{cc}
\frac{1-q^{-1}}{1-\chi_{\alpha}} \chi_{\alpha} & \mathbf{g}_{\psi^{-1}}((i-1) 4) \\
\mathbf{g}_{\psi^{-1}}((1-i) 4) & \frac{1-q^{-1}}{1-\chi_{\alpha}}
\end{array}\right) .
$$

It follows that for $2 \leq i \leq m$,

$$
\sigma_{\mathcal{O}_{i e}}^{\mathrm{Wh}}=\sigma_{\mathcal{O}_{i e}}^{\mathscr{X}}=\mathbb{1} \oplus \varepsilon,
$$

and

$$
\sigma_{\mathcal{O}_{(-m+1) e}}^{\mathrm{Wh}}=\sigma_{\mathcal{O}_{(-m+1) e}}^{\mathscr{X}}=\mathbb{1}
$$

however,

$$
\sigma_{\mathcal{O}_{e}}^{\mathrm{Wh}}=\varepsilon_{W}, \quad \sigma_{\mathcal{O}_{e}}^{\mathscr{X}}=\mathbb{1} .
$$

The last result on Whittaker dimension follows from Theorem 5.6.

One may also prove Proposition 8.3 by using the method described in $\S 5.4$. That is, we can compute $\operatorname{dim} \mathrm{Wh}_{\psi}\left(\pi_{\chi}^{u n}\right)$ by showing that

(i) Conjecture 6.4 holds for $\overline{\mathrm{SO}}_{3}$, and

(ii) we have

$$
\operatorname{rank}\left(\nu_{\mathfrak{R}_{i e}}^{\chi}\right)= \begin{cases}1 & \text { if } 2 \leq i \leq m \text { or } i=-m+1 \\ 0 & \text { if } i=1\end{cases}
$$

Here (i) can be verified exactly in the same way as Proposition 7.3, and thus we omit the details. We discuss (ii) for the three cases $i=-m+1, i=1$ and $2 \leq i \leq m$ separately.

- First, since $(-m+1) e=\rho-\rho_{Q, n}$, it is the unique element in $Y_{n}^{\text {exc }}$. In this case, the equality $\operatorname{rank}\left(\nu_{\mathfrak{R}_{(1-m) e}}^{\chi}\right)=1$ follows from Theorem 6.5.

- Second, for $\mathfrak{R}_{e}=\{e\}$, we have $\operatorname{rank}\left(\nu_{\mathfrak{R}_{e}}^{\chi}\right)=1$ if and only if $\mathcal{W}_{\mathbf{s}_{e}}^{*}\left(\mathbf{s}_{e}\right) \neq 0$. A straightforward computation from (32) gives that

$$
\mathcal{W}_{\mathbf{s}_{e}}^{*}\left(\mathbf{s}_{e}\right)=0 .
$$

Thus, $\operatorname{rank}\left(\nu_{\mathcal{O}_{e}}^{\chi}\right)=0$. 
- Third, we deal with free $W$-orbits $\mathcal{O}_{i e}, 2 \leq i \leq m$. Similar to the case of $\overline{\mathrm{SL}}_{2}^{(n)}$, we have $\operatorname{rank}\left(\nu_{\mathfrak{R}_{i e}}^{\chi}\right)=\operatorname{rank}\left(\mathcal{M}_{\mathfrak{R}_{i e}}\right)$ where

$$
\mathcal{M}_{\Re_{i e}}:=\left(\begin{array}{cc}
\mathcal{W}_{\mathbf{s}_{i e}}^{*}\left(\mathbf{s}_{i e}\right) & \mathcal{W}_{\mathbf{s}_{i e}}^{*}\left(\mathbf{s}_{(2-i) e}\right) \\
\mathcal{W}_{\mathbf{s}_{(2-i) e}^{*}}^{*}\left(\mathbf{s}_{i e}\right) & \mathcal{W}_{\mathbf{s}_{(2-i) e}}^{*}\left(\mathbf{s}_{(2-i) e}\right)
\end{array}\right) .
$$

Again, since ${ }^{w_{\alpha}} \chi=\chi$ and $\chi_{\alpha}=-1$, it follows from (32) that

$$
\mathcal{M}_{\Re_{i e}}=\left(\begin{array}{cc}
q^{(i-2) / 2} & q^{-(2+i) / 2} \mathbf{g}_{\psi^{-1}}((1-i) 4) \\
q^{i / 2} \mathbf{g}_{\psi^{-1}}((i-1) 4) & q^{-(2+i) / 2} .
\end{array}\right) .
$$

Note that we have $1 \leq i-1 \leq m-1$ and thus $\operatorname{det}\left(\mathcal{M}_{\mathfrak{R}_{i e}}\right)=0$. Clearly, this implies that $\operatorname{rank}\left(\mathcal{M}_{\mathfrak{R}_{i e}}\right)=1$.

Combining the above gives that $\operatorname{dim} \mathrm{Wh}_{\psi}\left(\pi_{\chi}^{u n}\right)=m$. It follows from this example of $\overline{\mathrm{SO}}_{3}^{(n)}$ that a naive analogue of Conjecture 5.7 does not hold for coverings of a general semisimple group. Here the difference between $\mathscr{X}_{Q, n}^{\mathrm{exc}}$ and $\left(\mathscr{X}_{Q, n}\right)^{W}$ plays a sensitive role and accounts for the failure. Indeed, in the case of $\overline{\mathrm{SO}}_{3}^{(n)}$, one has

$$
\left|\mathscr{X}_{Q, n}^{\mathrm{exc}}\right|=1 \text { and }\left|\left(\mathscr{X}_{Q, n}\right)^{W}\right|=2 \text {. }
$$

8.2. Double cover of $\operatorname{Spin}_{6}$. We consider in this subsection only double cover of $\operatorname{Spin}_{6} \simeq$ $\mathrm{SL}_{4}$, though the phenomenon appears for general $2 m$-fold cover of $\operatorname{Spin}_{2 k}$ with $m$ and $k$ being both odd. For this reason, we would like to consider the situation from the perspective of spin groups.

Consider the Dynkin diagram of simple coroots for the simply-connected $G=\operatorname{Spin}_{6}$ :

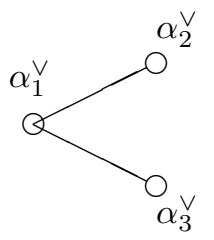

Let $Q$ be the Weyl-invariant quadratic form $Q$ of $Y$ such that $Q\left(\alpha_{i}^{\vee}\right)=1$ for all $1 \leq i \leq 3$. Let $\bar{G}$ be the double cover of $G$ arising from $Q$. One has $Y_{Q, 2}^{s c}=2 \cdot Y$ and

$$
Y_{Q, 2}=\left\{\sum_{i=1}^{3} y_{i} \alpha_{i}^{\vee}: 2 \mid y_{i} \text { for all } i \text { and } 2\left(y_{1}+y_{2}+y_{3}\right)\right\} \text {. }
$$

Thus, we get

$$
\bar{G}^{\vee}=\mathrm{SO}_{6}
$$

and that the principal endoscopic group $H$ for $\bar{G}$ is $\mathrm{SO}_{6}$. We have

$$
\mathscr{X}_{Q, 2}=\left\{0, \alpha_{1}^{\vee}, \alpha_{2}^{\vee}, \alpha_{1}^{\vee}+\alpha_{2}^{\vee}\right\} .
$$

Note that $\alpha_{2}^{\vee}=\alpha_{3}^{\vee} \in \mathscr{X}_{Q, n}$. There are two $W$-orbits of $\mathscr{X}_{Q, 2}$ represented by the following graph:

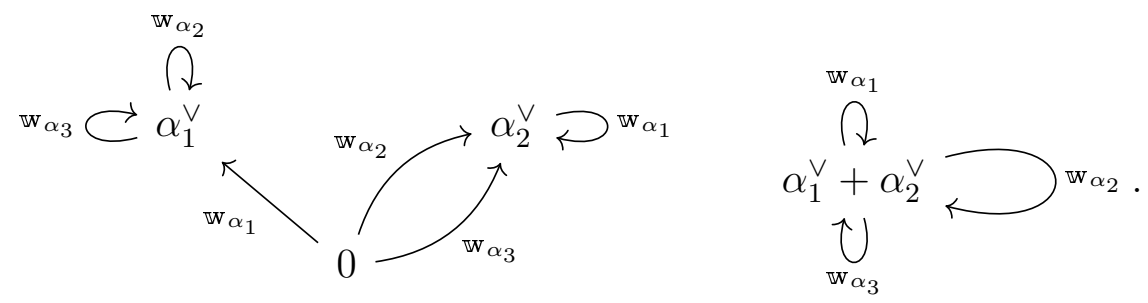

We have $\mathscr{X}_{Q, n}=\mathcal{O}_{0} \cup \mathcal{O}_{\alpha_{1}^{\vee}+\alpha_{2}^{\vee}}$. 
It then follows from [Gol94, Theorme 6.8] that the only nontrivial unramified $R_{\chi}$ is $\left\{1, \mathbb{w}=\mathbb{w}_{\alpha_{r-1}} \mathbb{W}_{\alpha_{r}}\right\}$ with

$$
\chi_{\alpha_{2}}=\chi_{\alpha_{3}}=-1 \text {. }
$$

A direct computation using (25), (26) and Theorem 5.2 gives that

$$
\begin{aligned}
\tau\left(w, \chi, \mathbf{s}_{0}, \mathbf{s}_{0}\right)+\tau\left(w, \chi, \mathbf{s}_{\alpha_{2}^{\vee}}, \mathbf{s}_{\alpha_{2}^{\vee}}\right) & =\gamma(w, \chi)-\gamma(w, \chi)=0 \\
\tau\left(w, \chi, \mathbf{s}_{\alpha_{1}^{\vee}}, \mathbf{s}_{\alpha_{1}^{\vee}}\right) & =\gamma(w, \chi) \\
\tau\left(w, \chi, \mathbf{s}_{\alpha_{1}+\alpha_{2}^{\vee}}, \mathbf{s}_{\alpha_{1}+\alpha_{2}^{\vee}}\right) & =-\gamma(w, \chi) .
\end{aligned}
$$

Denoting $\operatorname{Irr}\left(R_{\chi}\right)=\{\mathbb{1}, \varepsilon\}$, it then follows that

$$
\sigma_{\mathcal{O}_{0}}^{\mathrm{Wh}}=(2 \cdot \mathbb{1}) \oplus \varepsilon, \quad \sigma_{\mathcal{O}_{\alpha_{1}^{\vee}+\alpha_{2}^{\vee}}^{\mathrm{Wh}}}^{\mathrm{Wh}}=\varepsilon .
$$

In particular, writing $I(\chi)=\pi_{\chi}^{u n} \oplus \pi$, we have

$$
\operatorname{dim} \mathrm{Wh}_{\psi}\left(\pi_{\chi}^{u n}\right)=2, \text { and } \operatorname{dim} \mathrm{Wh}_{\psi}(\pi)=2 .
$$

On the other hand, it is clear from above graph that

$$
\sigma_{\mathcal{O}_{0}}^{\mathscr{X}}=3 \cdot \mathbb{1}, \quad \sigma_{\mathcal{O}_{\alpha_{1}^{\vee}+\alpha_{2}^{\vee}}^{\mathscr{X}}}^{\mathscr{V}}=\mathbb{1} .
$$

We see that analogous Conjecture 5.7 does not hold in this case. The constraint that $\bar{G}^{\vee}$ is of adjoint type seems to be necessary.

For a low-rank group and "small" $R_{\chi}$, one should be able to compute and verify explicitly Conjecture 5.7. However, for general $n$-fold covers of a simply-connected group $G$, in view of the difficulty highlighted in Remark 7.2, it is desirable to approach the problem from a more uniform and conceptual perspective. In any case, we will leave the investigation of this to a future work, as a continuation of the present paper.

\section{REFERENCES}

[Art84] James Arthur, On some problems suggested by the trace formula, Lie group representations, II (College Park, Md., 1982/1983), Lecture Notes in Math., vol. 1041, Springer, Berlin, 1984, pp. 1-49, DOI 10.1007/BFb0073144. MR748504

[Art89] Unipotent automorphic representations: conjectures, Astérisque 171-172 (1989), 1371. Orbites unipotentes et représentations, II. MR1021499

[Art93] - On elliptic tempered characters, Acta Math. 171 (1993), no. 1, 73-138, DOI 10.1007/BF02392767. MR1237898

[Art06] _ A note on L-packets, Pure Appl. Math. Q. 2 (2006), no. 1, Special Issue: In honor of John H. Coates., 199-217, DOI 10.4310/PAMQ.2006.v2.n1.a9. MR2217572

[Art13] _ The endoscopic classification of representations, Automorphic representations and Lfunctions, Tata Inst. Fundam. Res. Stud. Math., vol. 22, Tata Inst. Fund. Res., Mumbai, 2013, pp. 1-22, DOI 10.1090/coll/061/01. MR3156849

[BG12] Dubravka Ban and David Goldberg, R-groups and parameters, Pacific J. Math. 255 (2012), no. 2, 281-303, DOI 10.2140/pjm.2012.255.281. MR2928553

[BZ05] Dubravka Ban and Yuanli Zhang, Arthur R-groups, classical R-groups, and Aubert involutions for $\mathrm{SO}(2 n+1)$, Compos. Math. 141 (2005), no. 2, 323-343, DOI 10.1112/S0010437X04001113. MR2134270

[BM94] Dan Barbasch and Allen Moy, Whittaker models with an Iwahori fixed vector, Representation theory and analysis on homogeneous spaces (New Brunswick, NJ, 1993), Contemp. Math., vol. 177, Amer. Math. Soc., Providence, RI, 1994, pp. 101-105, DOI 10.1090/conm/177/01917. MR1303602

[Bor79] A. Borel, Automorphic L-functions, Automorphic forms, representations and $L$-functions (Proc. Sympos. Pure Math., Oregon State Univ., Corvallis, Ore., 1977), Proc. Sympos. Pure Math., XXXIII, Amer. Math. Soc., Providence, R.I., 1979, pp. 27-61. MR546608 
[Bou02] Nicolas Bourbaki, Lie groups and Lie algebras. Chapters 4-6, Elements of Mathematics (Berlin), Springer-Verlag, Berlin, 2002. Translated from the 1968 French original by Andrew Pressley. MR1890629

[BD01] Jean-Luc Brylinski and Pierre Deligne, Central extensions of reductive groups by $\mathbf{K}_{2}$, Publ. Math. Inst. Hautes Études Sci. 94 (2001), 5-85, DOI 10.1007/s10240-001-8192-2. MR1896177

[CS80] W. Casselman and J. Shalika, The unramified principal series of p-adic groups. II. The Whittaker function, Compositio Math. 41 (1980), no. 2, 207-231. MR581582

[CO13] Gautam Chinta and Omer Offen, A metaplectic Casselman-Shalika formula for $\mathrm{GL}_{r}$, Amer. J. Math. 135 (2013), no. 2, 403-441, DOI 10.1353/ajm.2013.0013. MR3038716

[GG18] Wee Teck Gan and Fan Gao, The Langlands-Weissman program for Brylinski-Deligne extensions, Astérisque 398 (2018), 187-275 (English, with English and French summaries). L-groups and the Langlands program for covering groups. MR3802419

[Gao17] Fan Gao, Distinguished theta representations for certain covering groups, Pacific J. Math. 290 (2017), no. 2, 333-379, DOI 10.2140/pjm.2017.290.333.

[Gao18] _ The Langlands-Shahidi L-functions for Brylinski-Deligne extensions, Amer. J. Math. 140 (2018), no. 1, 83-137, DOI 10.1353/ajm.2018.0001.

[Gaoa] - Hecke L-functions and Fourier coefficients of covering Eisenstein series, preprint, available at https://sites.google.com/site/fangaonus/research.

[Gaob] —, Kazhdan-Lusztig representations and Whittaker space of some genuine representations, Mathematische Annalen (2019, accepted), available at https://arxiv.org/abs/1903.06069.

[GSS] Fan Gao, Freydoon Shahidi, and Dani Szpruch, Local coefficients and gamma factors for principal series of covering groups, Memoirs of the AMS (2019, accepted), available at https://arxiv.org/abs/1902.02686.

[GW19] Fan Gao and Martin H. Weissman, Whittaker models for depth zero representations of covering groups, Int. Math. Res. Not. IMRN 11 (2019), 3580-3620, DOI 10.1093/imrn/rnx235. MR3961710

[Gin18] David Ginzburg, Non-generic unramified representations in metaplectic covering groups, Israel J. Math. 226 (2018), no. 1, 447-474, DOI 10.1007/s11856-018-1702-4. MR3819699

[Gol94] David Goldberg, Reducibility of induced representations for $\operatorname{Sp}(2 n)$ and $\mathrm{SO}(n)$, Amer. J. Math. 116 (1994), no. 5, 1101-1151, DOI 10.2307/2374942. MR1296726

[Gol11] _ On dual R-groups for classical groups, On certain L-functions, Clay Math. Proc., vol. 13, Amer. Math. Soc., Providence, RI, 2011, pp. 159-185. MR2767516

[KP84] D. A. Kazhdan and S. J. Patterson, Metaplectic forms, Inst. Hautes Études Sci. Publ. Math. 59 (1984), 35-142. MR743816

[Key82a] C. David Keys, On the decomposition of reducible principal series representations of p-adic Chevalley groups, Pacific J. Math. 101 (1982), no. 2, 351-388. MR675406

[Key82b] _ _ Reducibility of unramified unitary principal series representations of p-adic groups and class-1 representations, Math. Ann. 260 (1982), no. 4, 397-402, DOI 10.1007/BF01457019. MR670188

[Key87] _ L-indistinguishability and R-groups for quasisplit groups: unitary groups in even dimension, Ann. Sci. École Norm. Sup. (4) 20 (1987), no. 1, 31-64. MR892141

[KS88] C. David Keys and Freydoon Shahidi, Artin L-functions and normalization of intertwining operators, Ann. Sci. École Norm. Sup. (4) 21 (1988), no. 1, 67-89. MR944102

[KS75] A. W. Knapp and E. M. Stein, Singular integrals and the principal series. IV, Proc. Nat. Acad. Sci. U.S.A. 72 (1975), 2459-2461. MR0376964

[Lan76] Robert P. Langlands, On the functional equations satisfied by Eisenstein series, Lecture Notes in Mathematics, Vol. 544, Springer-Verlag, Berlin-New York, 1976. MR0579181

[LL79] J.-P. Labesse and R. P. Langlands, L-indistinguishability for SL(2), Canad. J. Math. 31 (1979), no. 4, 726-785, DOI 10.4153/CJM-1979-070-3. MR540902

[Li92] Jian-Shu Li, Some results on the unramified principal series of p-adic groups, Math. Ann. 292 (1992), no. 4, 747-761, DOI 10.1007/BF01444646. MR1157324

[Li12] Wen-Wei Li, La formule des traces pour les revêtements de groupes réductifs connexes. II. Analyse harmonique locale, Ann. Sci. Éc. Norm. Supér. (4) 45 (2012), no. 5, 787-859 (2013), DOI 10.24033/asens.2178 (French, with English and French summaries). MR3053009

[Li14] _ La formule des traces pour les revêtements de groupes réductifs connexes. I. Le développement géométrique fin, J. Reine Angew. Math. 686 (2014), 37-109, DOI 10.1515/crelle-2012-0015 (French, with French summary). MR3176600 
[Luo] Caihua Luo, Knapp-Stein dimension theorem for covering groups, preprint, available at https://sites.google.com/site/chluonus/research.

[McN12] Peter J. McNamara, Principal series representations of metaplectic groups over local fields, Multiple Dirichlet series, L-functions and automorphic forms, Progr. Math., vol. 300, Birkhäuser/Springer, New York, 2012, pp. 299-327, DOI 10.1007/978-0-8176-8334-413. MR2963537

[McN16] _ The metaplectic Casselman-Shalika formula, Trans. Amer. Math. Soc. 368 (2016), no. 4, 2913-2937, DOI 10.1090/tran/6597. MR3449262

[Pat87] S. J. Patterson, Metaplectic forms and Gauss sums. I, Compositio Math. 62 (1987), no. 3, 343-366. MR901396

[Sha83] Freydoon Shahidi, Some results on L-indistinguishability for $\mathrm{SL}(r)$, Canad. J. Math. 35 (1983), no. $6,1075-1109$, DOI 10.4153/CJM-1983-060-9. MR738845

[Sha90] _ A proof of Langlands' conjecture on Plancherel measures; complementary series for padic groups, Ann. of Math. (2) 132 (1990), no. 2, 273-330, DOI 10.2307/1971524. MR1070599

[Sha11] _ Arthur packets and the Ramanujan conjecture, Kyoto J. Math. 51 (2011), no. 1, 1-23, DOI 10.1215/0023608X-2010-018. MR2784745

[She79] D. Shelstad, Notes on L-indistinguishability (based on a lecture of R. P. Langlands), Automorphic forms, representations and $L$-functions (Proc. Sympos. Pure Math., Oregon State Univ., Corvallis, Ore., 1977), Proc. Sympos. Pure Math., XXXIII, Amer. Math. Soc., Providence, R.I., 1979, pp. 193-203. MR546618

[She82] — L-indistinguishability for real groups, Math. Ann. 259 (1982), no. 3, 385-430, DOI 10.1007/BF01456950. MR661206

[Sil78] Allan J. Silberger, The Knapp-Stein dimension theorem for p-adic groups, Proc. Amer. Math. Soc. 68 (1978), no. 2, 243-246, DOI 10.2307/2041781. MR0492091

[Sil79a] _ Correction: "The Knapp-Stein dimension theorem for p-adic groups" [Proc. Amer. Math. Soc. 68 (1978), no. 2, 243-246; MR 58 \#11245], Proc. Amer. Math. Soc. 76 (1979), no. $1,169-170$, DOI 10.2307/2042939. MR534411

[Sil79b] _ Introduction to harmonic analysis on reductive p-adic groups, Mathematical Notes, vol. 23, Princeton University Press, Princeton, N.J.; University of Tokyo Press, Tokyo, 1979. Based on lectures by Harish-Chandra at the Institute for Advanced Study, 1971-1973. MR544991

[Ste16] Robert Steinberg, Lectures on Chevalley groups, University Lecture Series, vol. 66, American Mathematical Society, Providence, RI, 2016.

[Szp15] Dani Szpruch, Symmetric genuine spherical Whittaker functions on $\overline{G S p_{2 n}(F)}$, Canad. J. Math. 67 (2015), no. 1, 214-240, DOI 10.4153/CJM-2013-033-5. MR3292701

[Szp19] _ On Shahidi local coefficients matrix, Manuscripta Math. 159 (2019), no. 1-2, 117-159, DOI 10.1007/s00229-018-1052-x. MR3936136

[Tat67] John T. Tate, Fourier analysis in number fields, and Hecke's zeta-functions, Algebraic Number Theory (Proc. Instructional Conf., Brighton, 1965), Thompson, Washington, D.C., 1967, pp. 305-347. MR0217026

[Wei09] Martin H. Weissman, Metaplectic tori over local fields, Pacific J. Math. 241 (2009), no. 1, 169-200, DOI 10.2140/pjm.2009.241.169. MR2485462

[Wei14] _ , Split metaplectic groups and their L-groups, J. Reine Angew. Math. 696 (2014), 89141, DOI 10.1515/crelle-2012-0111. MR3276164

[Wei18] _ _ L-groups and parameters for covering groups, Astérisque 398 (2018), 33-186 (English, with English and French summaries). L-groups and the Langlands program for covering groups. MR3802418

[Win78] Norman Winarsky, Reducibility of principal series representations of p-adic Chevalley groups, Amer. J. Math. 100 (1978), no. 5, 941-956, DOI 10.2307/2373955. MR517138

School of Mathematical Sciences, Yuquan Campus, Zhejiang University, 38 Zheda Road, Hangzhou, China 310027

E-mail address: gaofan.math@gmail.com 